\title{
VALIDATION OF AN EXERTIONAL RECALL QUESTIONNAIRE FOR A CIRCUIT TRAINING PROGRAM
}

\author{
by \\ Michael Gallagher, Jr. \\ B.S.E., University of Pittsburgh, 2003 \\ Submitted to the Graduate Faculty of \\ School of Education in partial fulfillment \\ of the requirements for the degree of \\ Masters of Science in Exercise Physiology
}

University of Pittsburgh

2007 


\section{UNIVERSITY OF PITTSBURGH \\ SCHOOL OF EDUCATION}

This thesis was presented

by

Michael Gallagher, Jr.

It was defended on

August 1, 2007

and approved by

Deborah J. Aaron, Ph.D., Department of Health and Physical Activity

Fredric L. Goss, Ph.D., Department of Health and Physical Activity

Kevin H. Kim, Ph.D., Department of Psychology in Education

Thesis Director: Robert J. Robertson, Ph.D., Department of Health and Physical Activity 


\title{
VALIDATION OF AN EXERTIONAL RECALL QUESTIONNAIRE FOR A CIRCUIT TRAINING PROGRAM
}

\author{
Michael Gallagher, Jr., M.S. \\ University of Pittsburgh, 2007
}

Physical activity recall questionnaires are important tools to quantify physical activity patterns and relating these patterns to disease risk. However, of the movement components that comprise physical activity, intensity is the most difficult to directly and objectively measure. PURPOSE: The present investigation examined the validity of a post-event recall questionnaire to measure the perception of physical exertion during a circuit exercise program. METHODS: Forty (21.1 \pm 2.7 yrs) recreationally active females $(\mathrm{n}=22)$ and males $(\mathrm{n}=18)$, with no prior knowledge of OMNI RPE, underwent a circuit program consisting of in counterbalanced order: (I) cycle ergometry at $50 \%$ and $75 \% \mathrm{VO}_{2 \text { peak }}$, and (II) bicep curls at $65 \%$ and $90 \% 1-\mathrm{RM}$. Seven days later, subjects rated their perceived exertion associated with each exercise bout using the Exertional Recall Questionnaire. The subjects reported OMNI RPEs for the overall body $(\mathrm{O})$, legs (L), and chest/breathing (C) for the cycle exercises, and OMNI RPEs for the overall body (O) and arm (A) for the bicep curl exercises. Following completion of the Exertional Recall Questionnaire, subjects were given standardized instructions for the OMNI RPE Cycle and Resistance Scales and repeated the circuit program. OMNI RPEs were then measured during the exercise bouts (the Criterion). RESULTS: Three-factor analyses of variance indicated that Recall and Criterion RPE-O, and -L did not differ significantly at both cycle exercise intensities $(\mathrm{p}=0.064$ and 0.169$)$. Recall RPE-C was significantly lower than Criterion RPE-C at both cycle 
exercise intensities $(\mathrm{p}=0.010)$. Three-factor analyses of variance indicated that the RPE-O and A for the Recall procedure were significantly less than the Criterion procedure $(\mathrm{p}<0.001)$ for both the lighter and heavier resistances. CONCLUSIONS: Recreationally active females and males with no prior knowledge of OMNI RPE were able to recall their perceived exertion (overall body and legs) experienced during non-weight bearing (cycle) aerobic exercise performed seven days previously. The Recall procedure is valid for non-weight bearing aerobic exercise. However, subjects under-estimated Recall RPE-O and -A for upper body resistance exercise. These responses question validity of the Recall RPE procedure for single joint arm resistance exercise. 
TABLE OF CONTENTS

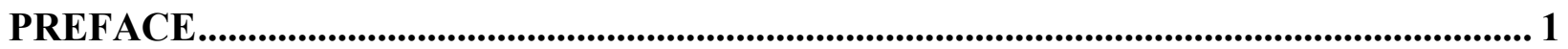

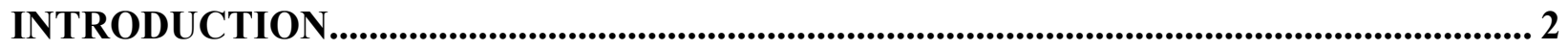

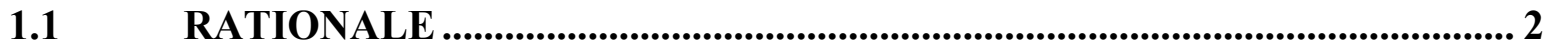

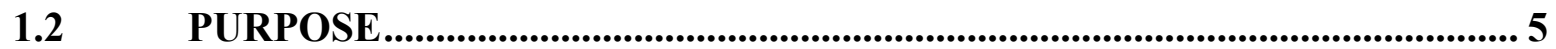

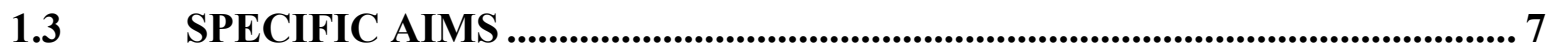

1.4 RESEARCH HYPOTHESES ............................................................................. 7

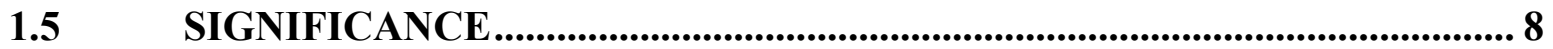

2.0 REVIEW OF RELATED LITERATURE ....................................................9

2.1 PHYSICAL ACTIVITY AND HEALTH .......................................................... 9

2.1.1 Current Recommendations........................................................................ 10

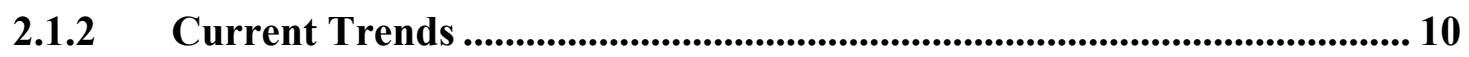

$2.2 \quad$ PHYSICAL ACTIVITY ASSESSMENT INSTRUMENTS ......................... 11

2.2.1 Direct Observation ............................................................................................ 12

2.2.2 Direct and Indirect Calorimetry.................................................................. 13

2.2.3 Doubly-Labeled Water ..................................................................... 13

2.2.4 Activity Monitors ............................................................................................ 14

2.2.5 Heart Rate Monitoring/Telemetry …………………………............................ 15

2.2.6 Self-Report Measures ...................................................................................... 15

2.3 RECALL QUESTIONNAIRES .................................................................... 16

2.3.1 Individualized Exercise Interventions: Targeting vs. Tailoring............... 18

2.4 RATINGS OF PERCEIVED EXERTION......................................................... 19

2.4.1 Borg's Effort Continua Model......................................................................... 20

2.4.2 Physiological Mediators of Perceived Exertion............................................... 21

2.4.3 Ratings of Perceived Exertion Scales .............................................................. 22 
2.4.4 Undifferentiated/Differentiated RPE ...................................................... 24

$2.5 \quad$ PUBLIC HEALTH APPLICATIONS .......................................................... 24

2.5.1 Exertional Observation ................................................................ 25

2.5.2 Exertional Session ................................................................................. 26

2.5.3 Exertional Recall .................................................................................. 27

2.5.4 Exercise Modes........................................................................................ 30

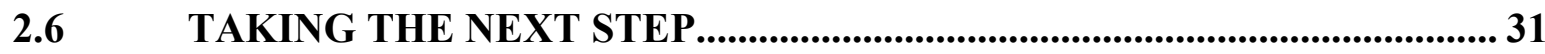

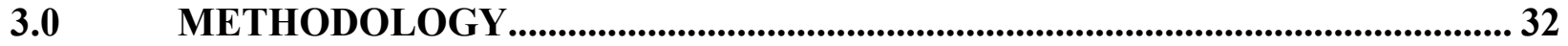

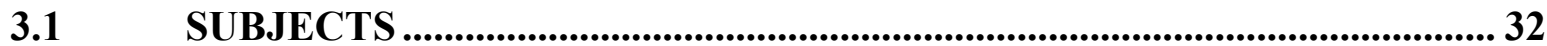

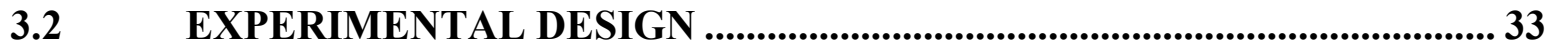

EXERCISE PROTOCOLS

3.3.1 Fitness Assessment Session........................................................................ 35

3.3.1.1 Aerobic Fitness............................................................................. 35

3.3.1.2 Biceps Muscle Strength .................................................................... 36

3.3.2 Circuit Exercise Session ........................................................................... 37

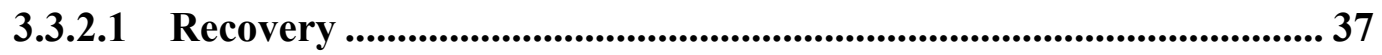

3.3.2.2 Cycling Exercise................................................................................ 37

3.3.2.3 Resistance Exercise - Biceps Curl..................................................... 38

3.3.3 Recall-Criterion Session ....................................................................... 39

3.3.3.1 Recall Questionnaire ............................................................................... 39

3.3.3.2 Ratings of Perceived Exertion - Exercise Measurement ................. 40

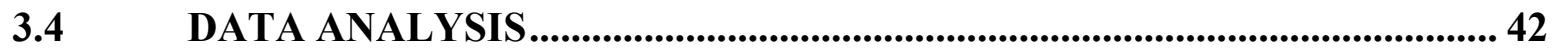

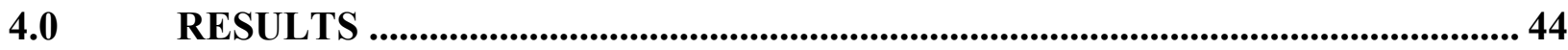

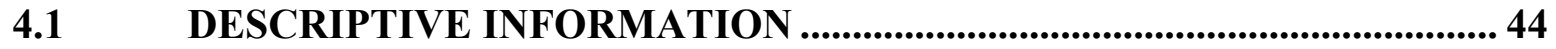

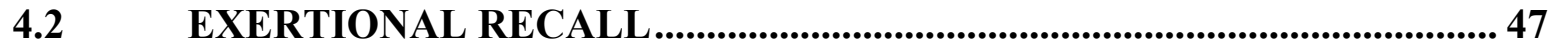

4.2.1 Cycling Exercise ........................................................................... 47

4.2.1.1 Overall Body Responses ........................................................................ 48

4.2.1.2 Leg Responses ............................................................................................ 49

4.2.1.3 Chest Responses ..........................................................................5 50

4.2.2 Resistance Exercise ......................................................................................... 51

4.2.2.1 Arm Responses...................................................................................... 52 
4.2.2.2 Overall Body Responses .......................................................................... 53

4.2.3 Circuit Program RPE-Session .................................................................... 53

4.3 ANALYSIS OF UNDER-ESTIMATION AND OVER-ESTIMATION ...... 54

4.3.1 Limits of Agreement for Cycle Exercise ................................................... 54

4.3.2 Limits of Agreement for Resistance Exercise................................................. 57

ANALYSIS OF COVARIANCE ...............................................................59

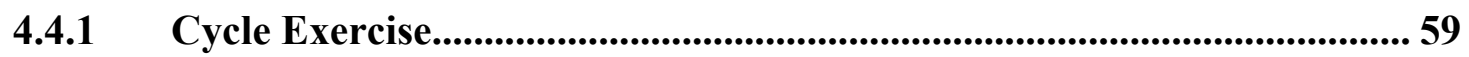

4.4.1.1 Overall Body Responses .............................................................................55

4.4.1.2 Leg Responses ................................................................................... 60

4.4.1.3 Chest Responses.............................................................................................. 61

4.4.2 Resistance Exercise ....................................................................................... 62

4.4.2.1 Arm Responses........................................................................................... 62

4.4.2.2 Overall Body Responses ............................................................................. 63

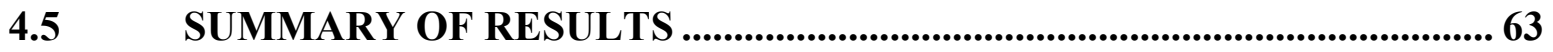

5.0 DISCUSSION, CONCLUSION, AND RECOMMENDATIONS......................... 65

5.1 RECALLED RPE: CYCLE EXERCISE ......................................................... 65

5.2 RECALLED RPE: RESISTANCE EXERCISE ........................................... 69

5.3 RECALLED RPE: SESSION........................................................................ 71

5.4 UNDERESTIMATION AND OVERESTIMATION_..................................... 72

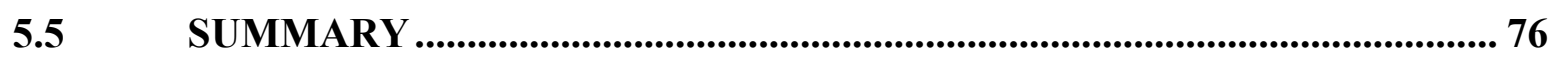

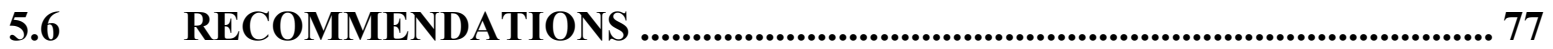

APPENDIX A - SUBJECT RECRUITMENT PACKET ........................................................... 80

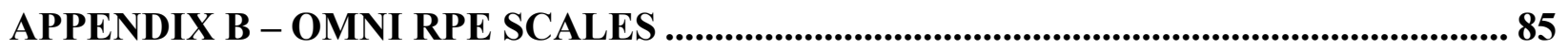

APPENDIX C - RECALL QUESTIONNAIRES ............................................................................8 88

APPENDIX D - OMNI SCALE INSTRUCTIONS ...................................................................... 92

APPENDIX E - MODIFIED ASTRAND PROTOCOL ................................................................ 95

APPENDIX F - MEAN RPE DESCRIPTIVES..................................................................97

APPENDIX G - PLOTS OF THE INTERACTIONS.............................................................. 99

APPENDIX H - BLAND-ALTMAN PLOTS OF STANDARDIZED RPE SCORES ....... 105

BIBLIOGRAPHY .............................................................................................................................. 116 


\section{LIST OF TABLES}

Table 1. Physical Activity Assessment Procedures and Characteristics.......................... 12

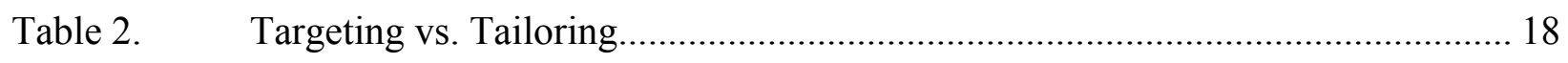

Table 3. Physiological Mediators of Perceived Exertion.................................................. 21

Table 4. Descriptive Characteristics of the Sample. Mean \pm SD ................................... 32

Table 5. Pearson Correlations between Recall and Criterion Ratings of Perceived

Exertion (RPE) for Cycle Exercise ..............................................................4

Table 6. Pearson Correlations between Oxygen Consumption and RPE ........................ 48

Table 7. Results of the Analysis of Variance for RPE-O during Cycle Exercise .............. 49

Table 8. Results of the Analysis of Variance for RPE-L during Cycle Exercise .............. 50

Table 9. Results of the Analysis of Variance for RPE-C during Cycle Exercise .............. 51

Table 10. Pearson Correlations between Recall and Criterion Ratings of Perceived

Exertion (RPE) for Resistance Exercise .....................................................51

Table 11. Results of the Analysis of Variance for RPE-A during Resistance Exercise ....... 52

Table 12. Results of the Analysis of Variance for RPE-O during Resistance Exercise ....... 53

Table 13. Frequency Distribution of the RPE Differences between Recall and

Criterion Procedures for Cycle Exercise* .....................................................57

Table 14. Frequency Distribution of the RPE Differences between Recall and

Criterion Procedures for Resistance Exercise*.................................................59

Table 15. Results of the Analysis of Covariance for RPE-O during Cycle Exercise ........... 60

Table 16. Results of the Analysis of Covariance for RPE-L during Cycle Exercise........... 61

Table 17. Results of the Analysis of Covariance for RPE-C during Cycle Exercise .......... 61

Table 18. Results of the Analysis of Covariance for RPE-A during Resistance Exercise ... 62

Table 19. Results of the Analysis of Covariance for RPE-O during Resistance Exercise ... 63 


\section{LIST OF FIGURES}

Figure 1. Borg's Effort Continua Model .................................................................... 20

Figure 2. Circuit Training Program Flow-Chart ........................................................... 34

Figure 3. Mean ( \pm SD) Recall and Criterion RPE during Cycle Exercise for Females ........45

Figure 4. Mean ( \pm SD) Recall and Criterion RPE during Resistance Exercise for

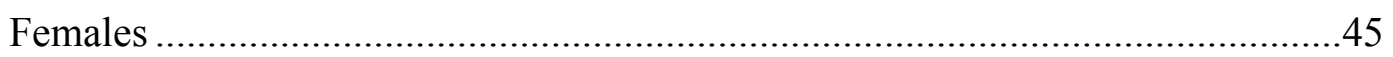

Figure 5. Mean $( \pm \mathrm{SD})$ Recall and Criterion RPE during Cycle Exercise for Males.......... 46

Figure 6. Mean ( \pm SD) Recall and Criterion RPE during Resistance Exercise for Males... 46

Figure 7. Bland-Altman Plots of (A) RPE-O, (B) RPE-L, and (C) RPE-C for Two

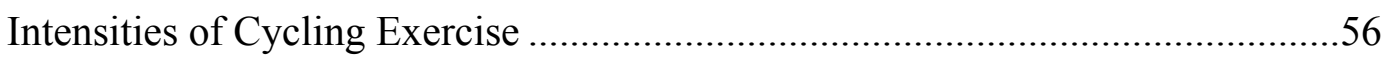

Figure 8. Bland-Altman Plots of (A) RPE-O and (B) RPE-A for Two Intensities of

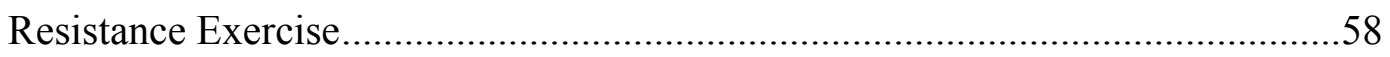

Figure 9. Reconfigured Bland-Altman Plots for Cycle Exercise .................................. 73

Figure 10. Reconfigured Bland-Altman Plots for Resistance Exercise ............................. 75 


\section{PREFACE}

I would like to thank Drs. Deborah Aaron, Fredric Goss, and Kevin Kim for serving on my thesis committee. Their guidance was invaluable in each of their specializations. I would like to extend a special thank you to my advisor and committee Chairperson, Dr. Robert Robertson. His patience and continued guidance allowed me to successfully complete my thesis whilst gaining a world of knowledge in research practices that has ultimately prepared me for the future.

I would also like to extend a heart-felt thank you to my family and friends for their diligence and support over these past years. I would not be here today if it was not for them. 


\subsection{INTRODUCTION}

This investigation validated a seven-day recall questionnaire to rate the perception of exertion (RPE) across multiple modes of physical activity. It was hypothesized that a recall questionnaire to rate the level of exertion experienced previously during cycling and resistance exercise is valid when examined in young, healthy adult females and males. Exertional recall is defined as the intensity of exertional perception experienced during physical activity and subsequently rated during a defined post-event period. Validation of an exertional recall procedure may lead to its inclusion in standard physical activity questionnaires, providing a perceptual measure of intensity for a specific physical activity.

\subsection{RATIONALE}

Standard physical activity recall questionnaires are important tools to quantify physical activity patterns and relate these to disease risk. Such information may then be used to develop healthy lifestyle intervention programs. However, of the movement components that comprise physical activity, intensity is the most difficult to directly and objectively measure. As such, the majority of standardized questionnaires assess physical activity intensity using estimated metabolic units (i.e. METS). The activities and their associated intensities reported in the questionnaire are assigned MET-values using previously published reference norms. These normative values are 
derived as population means for a specific type and intensity of physical activity. At best, they provide only approximations of actual physical activity intensity.

Reference standards for assessment of physical activity intensity include direct and indirect calorimetry, direct observation and doubly labeled water. These assessments provide measures of physical activity intensity through energy expenditure calculations. However, they are not consistently appropriate for large populations engaged in free-living or habitual exercise owing to extensive measurement burdens on the subject and the researcher. As such, these metabolic measures of physical activity have comparatively limited large scale public health applications. Additional measures of physical activity (i.e. intensity and volume) commonly used include heart rate telemetry, motion sensors, and activity logs.

An alternative means to measure physical activity intensity employs ratings of perceived exertion (RPE). Such perceptual measures can be included as part of a physical activity recall survey. Lee et al. ${ }^{1}$, reporting data from the Harvard Alumni Health Study, observed an inverse association between the relative intensity of physical activity as measured by recalled RPE and the level of disease risk. These data indicated that the beneficial health outcomes of physical activity were positively related to the relative exercise intensity as measured by RPE. When used in this context, the measure of relative exercise intensity using recalled RPE accounts for inter-individual differences in fitness level. An example of this measurement concept would be two individuals participating in an activity that requires 4 METS on the absolute scale. One person has a comparatively lower aerobic fitness level (6 METS maximal aerobic power) and responds with an RPE of 5. The other person has a higher aerobic fitness level (i.e. 8 METS) and responds with an RPE of 2. As the relative perceptual intensity is higher for the low fit person, he or she would gain more health benefit from participating in the 4 MET activities than the higher 
fit individual. The importance of this finding is that a given activity could be considered "low intensity" using absolute metabolic criterion but still provide health benefits based on its relative intensity as determined by the individual's level of perceived exertion. For a given MET requirement of the exercise intensity, an RPE estimate reflects inter-individual differences in maximal aerobic power. As such, at a given sub-maximal exercise MET level, the intensity of the perceptual signal varies inversely with $\mathrm{VO}_{2 \max }{ }^{1}$.

Lee et al. ${ }^{1}$ demonstrated that perceptual intensity varies directly with the relative metabolic rate. As such, those lower fit men who were not satisfying the current physical activity recommendations based on their reported participation in activities that had lower absolute intensities nevertheless received health benefits from exercise participation. The importance of a perceptually based relative intensity measure in physical activity questionnaires reflects mounting evidence that links both the quantity and quality of physical activity participation with life long health. The differences between the absolute and relative measures of exercise intensity suggest that 1) the recommendations need to be tailored to the individual and that global recommendations are not appropriate for all demographics, and 2) relative measures of exercise intensity may yield more accurate results of the physical activity behavior.

Use of the perceptual intensity level as determined by physical activity recall questionnaires acknowledges that fitness level should be considered in evaluating health outcomes of regular exercise participation prescriptions. Such application could alleviate confusion among physical activity recommendations as proposed by various governmental and academic sources while allowing a broader generalization of these recommendations to the population. These recommendations ${ }^{7-12}$ are typically standardized from normative data in order to apply to a large portion of the general population, thus the minimum exercise intensity and 
general metabolic demands are considered. Perceived exertion assessment places a low measurement burden on the exerciser and the investigator. Direct and absolute measures of intensity rely on normative assumptions and equipment. RPE assessment as part of a physical activity recall questionnaire requires minimal equipment and measurement time. This low subject and investigator burden allows for a greater application of the perceptual measurement to a larger population. However, there is limited published data on the validity of exertional recall to establish relative exercise intensities using questionnaire methodology.

\subsection{PURPOSE}

The present investigation examined the validity of a recall questionnaire to measure the perception of physical exertion experienced previously during standardized bouts of stationary cycling and resistance exercise for separate groups of female and male adults. This validation paradigm consisted of (a) one Fitness Assessment Session, (b) one Circuit Exercise Session, and (c) one Recall-Criterion Session. Each subject undertook the three sessions in the following sequence: Fitness Assessment, Circuit Exercise, and Recall-Criterion. Each of the three sessions was separated by exactly 7 days. RPE was measured using mode specific OMNI Scale pictorial formats ${ }^{2-4}$. Two different exercise modes (i.e. stationary cycling and biceps curl exercise) were performed at two different intensities. The intensity of the exercises for each mode differed between the first and second stations.

Aerobic fitness and muscle strength was measured during the Fitness Assessment Session. During the Circuit Exercise Session, the subjects did not estimate their RPE. During the Recall-Criterion Session, subjects were first asked to recall their RPE experienced during the 
circuit exercise performed 7-days previously. The Recall RPE was determined using the 7-day exertional recall questionnaire. Subsequent to filling out the questionnaire, the subjects performed the exercise circuit with RPE actually measured.

Ratings of perceived exertion for the overall body (RPE-O), legs (RPE-L) and chest/breathing (RPE-C) during stationary cycling were assessed using the recall questionnaire that employed the OMNI-Cycle Scale. During biceps curl exercise, RPE for the overall body (RPE-O) and dominant arm (RPE-A) were assessed using the recall questionnaire formatted with the OMNI-Resistance Exercise Scale. These RPE measures were termed the recall variables. The RPE-O, RPE-L, and RPE-C were directly measured during cycling exercise, and RPE-O and RPE-A were directly measured during resistance exercise. These RPE measures served as the criterion variables.

It is important to note that use of RPE as a perceptual estimation of the relative exercise intensity "accurately distinguishes between intensities." An exertional measure can then be used to profile varying intensities that are undertaken with the same exercise mode in a single conditioning session such as is typical of the physical activity patterns frequently reported in recall questionnaires. In the case of stationary cycling, the intensities span the metabolic range to incorporate exercise below and above the ventilatory threshold. The intensities for the resistance exercises (the "lighter" and the "heavier" weights) are intensities typically employed in resistance exercise programs for enhancing both muscular endurance and strength, respectively. 


\subsection{SPECIFIC AIMS}

The specific aims of this study were:

1. To determine if the perception of physical exertion (i.e. RPE-O, RPE-L, and RPE-C) experienced during the cycling portion of the Circuit Exercise Session and recalled 7-days later does not differ from the RPE rated by the subject during the RecallCriterion Session employing the same cycling intensity and duration.

2. To determine if the perception of physical exertion (i.e. RPE-O and RPE-A) experienced during the resistance component of the Circuit Exercise Session and recalled 7-days later does not differ from the RPE rated by the subject during the Recall-Criterion Session employing the same resistance intensities.

3. To determine if the perception of physical exertion (i.e. RPE-Session) experienced for the entire Circuit Exercise Session and recalled 7-days later does not differ from the RPE-Session rated by the subject during the Recall-Criterion Session employing the same circuit protocol.

\subsection{RESEARCH HYPOTHESES}

1. It was hypothesized that perception of physical exertion (i.e. RPE-O, RPE-L, and RPE-C) experienced during the cycling portion of the Circuit Exercise Session and recalled 7-days later would not differ from the RPE rated by the subjects during the Recall-Criterion Session employing the same cycling intensity and duration. 
2. It was hypothesized that perception of physical exertion (i.e. RPE-O and RPEA) experienced during the resistance component of the Circuit Exercise Session and recalled 7-days later would not differ from the RPE rated by the subjects during the Recall-Criterion Session employing the same resistance intensities.

3. It was hypothesized that perception of physical exertion (i.e. RPE-Session) experienced during the entire Circuit Exercise Session and recalled 7-days later would not differ from the RPE-Session rated by the subjects during the Recall-Criterion Session employing the same circuit protocol.

\subsection{SIGNIFICANCE}

Use of the perceptual intensity level as determined by a physical activity recall questionnaire could provide valuable information in evaluating health outcomes of regular exercise participation prescriptions. Such application could alleviate confusion among physical activity recommendations as proposed by various governmental and academic sources while allowing a broader generalization of these recommendations to the population.

The importance of a perceptually based relative intensity measure in physical activity questionnaires reflects mounting evidence that links both the quantity (volume) and quality (intensity) of physical activity participation with life long health. However, there is limited published data on the validity of exertional recall to establish relative exercise intensities using questionnaire methodology. 


\subsection{REVIEW OF RELATED LITERATURE}

\subsection{PHYSICAL ACTIVITY AND HEALTH}

The importance of physical activity in promoting an individual's health was documented in the 1979 Surgeon General's report titled Healthy People ${ }^{5}$. This report served as a guideline to promote health and prevent illness, disability, and premature death by increasing daily physical activity ${ }^{5}$. In the past twenty years, regular physical activity has been promoted as a primary component of a healthy lifestyle and the importance of these health benefits have gained momentum in the presence of the alarming rise in overweight and obese adults and children in the United States.

Most individuals can benefit from physical activity, not just those who are overweight and obese. Physical activity has been shown to promote healthy bones and muscles, increase lean muscle, reduce feelings of depression and anxiety, reduce body fat, control body weight, reduce blood pressure, and improve blood glucose control ${ }^{5-9}$. As a result, physical activity decreases the risk of chronic and degenerative diseases such as coronary heart disease, some cancers, high blood pressure, diabetes, and osteoporosis. 


\subsubsection{Current Recommendations}

In an effort to increase physical activity and to alleviate confusion as to optimal levels of physical activity participation, government agencies, health organizations and academic institutions have proposed physical activity guidelines to promote health benefits. Healthy People 2010, a comprehensive nationwide health promotion and disease prevention agenda, provides a strategy for improving the health of all people in the United States ${ }^{8}$. The Centers for Disease Control and Prevention (CDC) and the American College of Sports Medicine (ACSM) recommend adults should either engage in moderate-intensity physical activities for at least 30 minutes on 5 or more days of the week or in vigorous-intensity physical activity for 20 or more

minutes 3 or more days per week ${ }^{9,10}$. The American Heart Association (AHA) recommends that adults should exercise for 30 to 60 minutes, at 50-80 percent of maximum capacity on most days of the week, to achieve a moderate health and fitness level ${ }^{11}$. The American Diabetes Association (ADA) recommends adults with Type II diabetes engage in physical activities for 20-60 minutes at an intensity equivalent to $50-70 \%$ of maximum aerobic power 3 to 5 days a week $^{12}$. Even though these recommendations appear useful, they are subject to periodic change secondary to scientific and medical input, and the content discrepancies between agencies and institutions can compound interpretation and implementation.

\subsubsection{Current Trends}

According to the Healthy People reports and the CDC's National Health Interview Survey (NHIS), poor diet and physical inactivity accounted for an estimated 17 percent of the deaths in the United States in 2000. Additionally, it was found that (a) approximately 19 percent of U.S. 
adults engage regularly ( 3 times a week for at least 20 minutes) in vigorous physical activity during leisure time that involves large muscle groups in dynamic movement, (b) 23.5 percent of adults engage regularly (5 times a week for at least 30 minutes) in sustained physical activity of any intensity during leisure time, (c) 25 percent of adults report no leisure physical activity, and (d) 60-75 percent of adults report no regular physical activity ${ }^{8,9}$. Preliminary data show a nominal increase, from 32 percent in 1997 to 33 percent in 2003, of adults who engaged regularly in moderate physical activity (i.e., $30+$ minutes of moderate activity at least 5 times a week) ${ }^{8}$. In regard to the alarming obesity and overweight epidemic, the proportion of obese adults in the United States increased from 23 to approximately 30 percent.

\subsection{PHYSICAL ACTIVITY ASSESSMENT INSTRUMENTS}

There is a wide range of instruments and measurement techniques to quantify physical activity levels, patterns, and behavior, and to determine physical activity intensity (Table 1). Some assessment tools are considered first choices or the "gold standard." Each type of measuring tool is particular to a specific need or environment and thus most techniques are inadequate for assessing physical activity in free-living conditions across broad segments of the population where both conditioning outcomes and health benefits vary widely. 
Table 1. Physical Activity Assessment Procedures and Characteristics

\begin{tabular}{|l|c|c|c|c|c|c|c|c|c|c|}
\hline & & Use in & & & Low & Low & & & \\
Measurement & Large & Low & Low & Subject & Subject & Likely to & & \\
Tool & Applicable & Scale & $\$$ & Time & Time & Effort & Influence & Acceptable & Socially & Activity \\
& Age Groups & Studies & Cost & Cost & Cost & Cost & Behavior & to Individuals & Acceptable & Specific \\
\hline
\end{tabular}

\begin{tabular}{|c|c|c|c|c|c|c|c|c|c|c|}
\hline Task Specifie Diary & adult, elderly & yes & yes & yes & no & no & yes & mayte & yes & yes \\
\hline Fecall Questionmaire & atult, elderly & yes & yes & yes & $y=s$ & $y=$ & no & yes & yes & wes \\
\hline Ouantitative History & adult, elderly & yes & yes & no & no & no & no & yes & yes & yes \\
\hline Global Self-Report & atult, elderly & yes & yes & yes & yes & yes & no & yes & yes & no \\
\hline
\end{tabular}

\begin{tabular}{|c|c|c|c|c|c|c|c|c|c|c|}
\hline Monitoring & & & & & & & & & & \\
\hline Observation & all & no & no & no & no & yes & yes & mayte & maybe & yes \\
\hline Heart Rate Moritor & all & no & no & no & yes & yes & no & yes & yes & no \\
\hline Fedorneter & atult, elderly & yes & yes & yes & yes & yes & no & yes & yes & no \\
\hline Accelerometer & all & yes & yes & yes & yes & yes & no & yes & yes & no \\
\hline Direct Cilorimstry & all & no & no & no & no & no & yes & no & no & yes \\
\hline Indinect Calorinetry & adult, elderly & no & no & no & no & no & yes & no & no & yes \\
\hline Donbly Labueled Water & all & no & no & no & yes & yes & no & yes & yes & no \\
\hline
\end{tabular}

\subsubsection{Direct Observation}

Direct observation is a strong criterion measure of physical activity. A criterion measurement can be used to validate other forms of physical activity assessments. Direct observation primarily measures the behavioral component of free-living physical activity. This form of assessment has a high investigator burden. It's labor and time intensive process is necessary for measurement accuracy and typically used in adult studies and children within a small sample ${ }^{13,14}$. It is proposed that direct observation ensures a comparatively more conclusive record of physical activity behavior patterns. Typically, a trained observer will measure the frequency, duration, and type of activity at given time intervals. An example of this method would be an observer recording what types of activity a child performed during recess and for how long or how many times it was performed. 


\subsubsection{Direct and Indirect Calorimetry}

One of the more widely used criterion estimation measures of physical activity intensity is the use of calorimetry whether it employ direct or indirect methodology. Calorimetry provides an estimate of physical activity intensity using calculated aerobic energy expenditure. Direct calorimetry has a higher financial and time cost than indirect calorimetry but yields more

accurate measurements ${ }^{13}$. Direct calorimetry places an individual in an air sealed room. The airflow into the room and its percentage of oxygen and carbon dioxide are standardized. The airflow out of the room and its percentage of oxygen and carbon dioxide are measured. The inspired and respired fractional equivalence of oxygen and carbon dioxide in air are. These values are then converted to caloric values using energy turnover as determined by the respiratory exchange ratio. Indirect calorimetry, on the other hand, has a greater accessibility and economic feasibility for researchers and subjects ${ }^{13,14}$. The instruments used with indirect calorimetry use respiratory gas analysis to calculate oxygen consumption and in turn to measure aerobic energy expenditure of the performed work or activity.

\subsubsection{Doubly-Labeled Water}

One of the most accurate measures of physical activity energy expenditure is the doubly-labeled water (DLW) method, often referred to as a criterion or "gold standard" measure ${ }^{13,14}$. DLW is a biochemical procedure used to estimate the total daily energy expenditure through biological tracers that indicate the rate of metabolism by measuring carbon dioxide production. Even though this method of assessing physical activity is considered to be one of the most accurate, it is typically used for smaller sample sizes or used in conjunction with other modes of assessment 
such as indirect calorimetry or physical activity logs. Both the isotope tracers and the analysis procedure are costly. However, DLW facilitates energy expenditure measurements of free-living and habitual exercise ${ }^{13}$.

\subsubsection{Activity Monitors}

Use of activity monitors has increased in popularity in recent decades, particularly in the last ten years owing to technological advancements and reduced production costs. Activity monitors are an inexpensive, cost-effective, and easy to use means of objectively measuring free-living physical activity through movement counts and can be used to estimate energy expenditure of the

activity ${ }^{13,14}$. The two main types of activity monitors are pedometers and accelerometers. Pedometers are typically used to measure walking and walking-based activities as this mode of activity is a normal and common daily event involving linear movement. Step counts or distance traveled derived from the pedometer can be used as an estimate of energy expenditure through calibration equations or previously established normative data. However, step counts are not a sensitive measure of physical activity intensity. Pedometers are unable to adequately recognize or record "non-linear" forms of activity such as stationary cycling, resistance training, swimming, or even events performed on inclined surfaces ${ }^{13}$. A technically more complex form of a pedometer is the accelerometer. Accelerometers are technically similar to pedometers but they can assess acceleration in either one plane or multiple planes. The placement and location of the accelerometer influences the instrument's ability to accurately record movement. This inability to accurately measure the acceleration of the body in all appropriate planes of movement is only one of the limitations of accelerometer use. Other limitations are similar to that of pedometers ${ }^{13,14}$. 


\subsubsection{Heart Rate Monitoring/Telemetry}

Another popular method to assess physical activity is the use of a heart rate monitor. This form of monitoring is based on the association between heart rate and the aerobic energy expenditure required for a specific amount of physical activity. Exercise-induced heart rate response provides an indication of the relative stress placed on the cardio-respiratory system ${ }^{15,16}$. In general, heart rate is positively related to oxygen uptake and is a major determinant of cardiac output particularly at moderate to near maximal exercise intensities ${ }^{15}$. An increase in heart rate contributes to increasing cardiac output. The increase in cardiac output is directly proportional to the increase in the delivery of oxygen to the active muscles engaged in the physical activity ${ }^{15}$. The primary disadvantage to heart rate monitoring involves the impact of extraneous factors on cardiac frequency such as psychological stress, sleep behavior, medication, and an unaccustomed environment such as a laboratory ${ }^{13}$. Additionally, heart rate monitoring is somewhat less accurate in sedentary individuals and at low physical activity intensity levels ${ }^{13,14}$.

\subsubsection{Self-Report Measures}

Self-report measures of physical activity remain one of the most commonly used instruments in research studies and public health screenings. The financial and participant burden of these procedures is low as the individual directly provides the information about his or her qualitative and quantitative physical activity patterns and behavior ${ }^{13}$. Self-report measurements are typically obtained by a comparatively-short questionnaire. The main types of self-report measures are physical activity diaries or logs, quantitative history questionnaires, and recall questionnaires 13, 14. Examples of physical activity diaries or logs are the Bouchard 3-Day 
Physical Activity $\log { }^{17}$ and such public health recall instruments as the Paffenbarger ${ }^{18}$ and the CARDIO $^{19}$ physical activity questionnaires. However, many of these self-report purports to measure physical activity intensity using absolute measures of energy expenditure, such as an estimated metabolic equivalent (MET) value for a specific activity ${ }^{20}$. The derived physical activity energy expenditure is the estimated MET value for the engaged physical activities. The total energy expenditure number (movement counts, Kcals per day) is compared with previously established normative data to rank that individual according to health and physical activity standards. Studies have found large variability $(r=-0.10$ to 0.88$)$ in the correlation coefficients between self-report physical activity measures and direct observation, DLW, heart rate, or pedometer measures ${ }^{13,23,25,26}$. Particularly, the correlations for 7-day physical activity recall questionnaires $(\mathrm{r}=0.5$ to 0.56$)$ were higher than the correlations for global measures of physical activity $(r=0.14$ to 0.36$){ }^{13}$. However, the validity of self-report questionnaires is negatively affected by recall errors, deliberate misinterpretation, and social desirability ${ }^{13,14}$.

\subsection{RECALL QUESTIONNAIRES}

Recall questionnaires are a specific type of self-report that uses a range of response epochs varying from one day up to a year. The participant is asked to recall the intensity and the duration of physical activities in which they engaged during the specified epoch ${ }^{13,21}$. The respondent's data are objectively classified into general activity behaviors such as active or inactive. Recall questionnaires are commonly used because they are appropriate for a wide range of environments having differing population subsets and for many physical activities. Particular benefits include applicability in large-scale epidemiology studies, low cost, and low investigator- 
subject burden ${ }^{13,14}$. The disadvantage of a self-report survey is recall bias on the part of the participant. It has been demonstrated that physical activity questionnaires both over and underestimate the total amount of physical activity. Duncan et al. ${ }^{21}$ found that when using a recall questionnaire, sedentary adults overestimate the intensity of their activity particularly when the activity is moderate intensity. Additionally, Strath et al. ${ }^{23}$ found that there is reduced accuracy in recall reporting for light to moderate intensity activities and total physical activity was underestimated as compared to heart rate and motion assessments of physical activity.

The majority of, if not all, recall questionnaires use an absolute intensity scale based on MET values derived from normative data to estimate the intensity of physical activities. This method to assess physical activity intensity produces a generalized estimation of physical activity intensity which may be appropriate for large scale epidemiological studies. However, the method has limitations in determining the physical activity level of an individual or a small cohort. The measures of absolute and relative intensities have "substantial" inter- and intraindividual variability depending on age, sex, skill, level of fatigue, and body mass and surface area ${ }^{1,13}$. The relative metabolic intensity (i.e. $\% \mathrm{VO}_{2 \max }$ ) of a given physical activity is a function of the individual's aerobic fitness level.

The use of physical activity recall questionnaires is considered to be valid and reliable within the research context ${ }^{22-29}$. However, the absolute measures of physical activity intensity may affect the prevalence estimates for physical activity guidelines and conformity, and may be inaccurate for habitual exercise behavior ${ }^{30,31}$. Use of a relative measure of physical activity may provide more accurate physical activity information because the respondent data is determined individually. 


\subsubsection{Individualized Exercise Interventions: Targeting vs. Tailoring}

Existing evidence supports a relative intensity measure of physical activity in exercise and weight management intervention programs, and in exercise prescription. These programs focus on the importance of relative measures of exercise intensity in both prescribing and selfregulating physical activity programs.

The "targeting or tailoring" approach to exercise prescription is one such example. Research in human behavior has shown the individualized exercise interventions are more effective than the commonly used "one-size-fits-all" approach which tries to accommodate a large portion of the population through a generalized conditioning prescription ${ }^{33,34}$. Targeting and tailoring exercise programming has gained popularity as these approaches try to customize the interventions and information to the studied population. Targeting is based on a common characteristic within the population cohort whereas tailoring is more specific to the individual ${ }^{33}$. The differences between the two approaches are generally found in the assessment method and the cost to both the investigator and the participant (Table 2).

Table 2. Targeting vs. Tailoring

\begin{tabular}{lll}
\hline & \multicolumn{1}{c}{ Targeted } & \multicolumn{1}{c}{ Tailored } \\
\hline $\begin{array}{l}\text { Amount of assessment } \\
\text { Level of personalization }\end{array}$ & Low/moderate & High \\
$\begin{array}{l}\text { Participant time/burden } \\
\text { Ability to reach large numbers }\end{array}$ & Moderate & High \\
$\begin{array}{l}\text { Ability to address unique or } \\
\text { individual characteristics }\end{array}$ & High & $\begin{array}{l}\text { Moderate/high } \\
\text { Moderate/high } \\
\text { Assumption that individuals who } \\
\text { share a characteristic are } \\
\text { homogenous on other factors }\end{array}$ \\
$\begin{array}{l}\text { Cost (implementation) } \\
\text { Cost (development) }\end{array}$ & High & Low/moderate \\
\hline
\end{tabular}

Napolitano MA and Marcus BH. Targeting and tailoring physical activity information using print and information technologies. Exer Sport Sci Rev. Vol. 30(3): 122-128. 2002 
The use of both targeting and tailoring exercise programming procedures makes it possible to undertake large-scale interventions employing more individualized prescriptive approach ${ }^{33}$. Research has demonstrated the potential benefits of these techniques, especially tailoring ${ }^{33-36}$. Bull et al. ${ }^{37}$ found that patients in the tailored group were more likely to increase their level of physical activity than patients in the personalized (i.e. exercise prescription designed for the patient based on their abilities, needs, and likes), general, and control groups. From these intervention studies, it is reasonable that a more customized approach to self-report physical activity questionnaires such as the use of relative intensity will provide a stronger physical activity characteristic for the populations.

\subsection{RATINGS OF PERCEIVED EXERTION}

Ratings of perceived exertion (RPE) are perceptual indicators of the physical strain and associated subjective effort during dynamic and resistance exercise and provide a quantitative measure to track and prescribe physical activity and exercise conditioning ${ }^{2,13,15}$. The concept and measurement of exertional perceptions was developed and validated by Gunnar Borg ${ }^{15,} 32$. Borg's work in the late 1950's and early 1960's demonstrated a consistent positive relation between heart rate, oxygen consumption, and ratings of perceived exertion (RPE) during load incremented protocols involving various modes of aerobic exercise. This perceptualphysiological congruence formed the conceptual basis of "Borg's Effort Continua Model" (Figure 1). 


\section{Effort Continua}

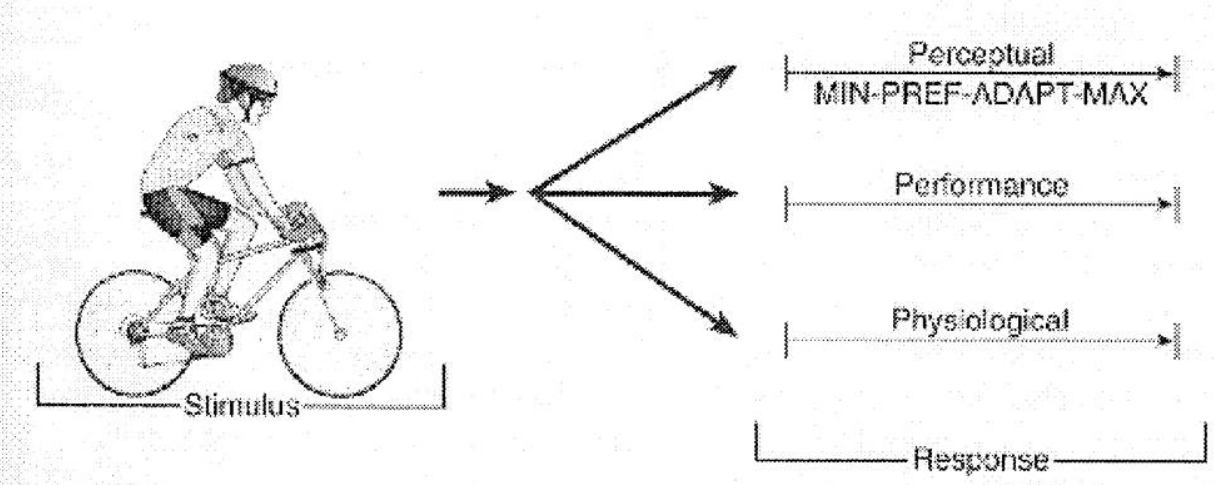

Figure 1. Borg's Effort Continua Model

Robertson RJ. Perceived Exertion for Practitioners: Rating Effort with the OMNI Picture System. Champaign, IL: Human Kinetics. 2004

\subsubsection{Borg's Effort Continua Model}

The model proposes that the responses to exercise involve physiological, perceptual, and performance components that form an effort continuum. The relation between the physiological demands of exercise performance and the perception of exertion associated with the exercise performance forms a functional linkage that serves as the rationale for Borg's Effort Continua Model. As intensity of the exercise performance increases, there is a corresponding and interdependent change in both the perceptual and physiological processes of the body ${ }^{2,15,32}$. It is important to note that the validation of the perceptual-physiological link described by the effort continua model is essential in the application of ratings of perceived exertion for sports, clinical, and public health settings. 


\subsubsection{Physiological Mediators of Perceived Exertion}

There are three classes of perceptual signals associated with physiological mediators: respiratory-metabolic, peripheral, and non-specific. Respiratory-metabolic mediators influence ventilatory drive during exercise. Peripheral mediators are localized in the limbs and the trunk and involve alterations in energy production and contractile properties of skeletal muscle. Nonspecific mediators include general or systemic physiological responses associated with exercise (Table 3). ${ }^{2}$

Table 3. Physiological Mediators of Perceived Exertion

\begin{tabular}{|c|c|c|}
\hline $\begin{array}{l}\text { Respiratory- } \\
\text { Metabolic }\end{array}$ & Peripheral & Non-Specific \\
\hline Pulmonary Ventilation & $\begin{array}{l}\text { Metabolic Acidosis } \\
\text { (pH, Lactic Acid) }\end{array}$ & $\begin{array}{l}\text { Hormonal Regulation } \\
\text { (catecholamines, } \\
\beta \text {-endorphins) }\end{array}$ \\
\hline Oxygen Uptake & Blood Glucose & $\begin{array}{l}\text { Temperature } \\
\text { Regulation } \\
\text { (core and skin) }\end{array}$ \\
\hline $\begin{array}{l}\text { Carbon Dioxide } \\
\text { Production }\end{array}$ & Blood Flow to Muscle & Pain \\
\hline Heart Rate & Muscle Fiber Type & Cortisol and Serotonin \\
\hline Blood Pressure & Free Fatty Acids & $\begin{array}{l}\text { Cerebral Blood Flow } \\
\text { and Oxygen }\end{array}$ \\
\hline & Muscle Glycogen & \\
\hline
\end{tabular}

One of the proposed physiological mediators of the respiratory-metabolic perceptual signal of exertion is total body oxygen uptake measured in absolute (i.e. L/min) and relative (i.e. $\left.\% \mathrm{VO}_{2 \max }\right)$ units. As previously mentioned in conjunction with Borg's Effort Continua Model, there is a functional link between the physiological demands of exercise and perceptual signals of exertion. The perceptual signals associated with oxygen uptake, are mediated through ventilatory drive required to support aerobic metabolism ${ }^{2}$. As the ventilatory drive increases in response to greater aerobic energy requirements, the increase in developed inspiratory muscle 
tension is consciously perceived as a signal of respiratory-metabolic exertion ${ }^{2,15}$. During dynamic exercise, the correlation between $\mathrm{VO}_{2}$ and RPE ranges from 0.76 to $0.97^{2,15}$. However, a number of investigations observed poor correspondence between oxygen uptake and RPE. This occurred when oxygen uptake was expressed in absolute units (i.e. $1 \cdot \mathrm{min}^{-1}$ or $\mathrm{ml} \cdot \mathrm{kg}^{-1} \cdot \mathrm{min}^{-1}$ ). In contrast, it was determined that the role of aerobic metabolism in mediating perceived exertion is more clearly defined through the use of relative oxygen uptake $\left(\% \mathrm{VO}_{2 \max }\right)^{2}$. The relation between relative oxygen uptake and RPE is important as a metabolic marker for different types of exercise modes.

The direct linear relationship between $\mathrm{RPE}$ and $\mathrm{VO}_{2}$ is the central component of a concurrent paradigm to validate a perceived exertion metric. As the intensity of the exercise increases, ventilatory drive increases as well as oxygen consumption. The increase in $\mathrm{VO}_{2}$ yields a corresponding and parallel increase in RPE. Low intensity exercise results in a lower $\mathrm{VO}_{2}$ with RPEs in the lower zone (OMNI-RPE $=1$ to 3$)$. High intensity exercise results in a higher $\mathrm{VO}_{2}$ with RPEs in the higher zone (OMNI-RPE $=7 / 8$ to 10$)$. Examining the correlation between the respiratory-metabolic response and the perceptual response enables validation of perceived exertion in new application dimensions such as exertional recall.

\subsubsection{Ratings of Perceived Exertion Scales}

The original 15-category Borg RPE scale was assumed to be directly associated with exercise heart rate as determined in young, healthy male adults ${ }^{15,32}$. As such, the scale contained numerical response categories from 6 to 20 that were expected to correspond to measured heart rate ranging from 60 to $200 \mathrm{bpm}$. This $\mathrm{RPE}$ ratio $(\mathrm{HR}=\mathrm{RPE} \times 10)$ has limited validity as heart rate is inherently dependent on age, gender, exercise mode, and environment causing marked 
inter- and intra-individual variability in the RPE ratio. However, the 15-category Borg RPE scale retains functionality through the interval properties of the scale in regard to the linear relationship between heart rate and the perceptual ratings ${ }^{15}$. It was reasoned that heart rate is one of the primary measures of physiological strain during aerobic exercise providing the empirical basis to develop and validate a perceptual rating scale with heart rate as the measurement criterion. A number of studies have validated both Borg's 15-category scale and the 10-categoryratio scale using oxygen uptake $\left(\mathrm{VO}_{2}\right)$ and heart rate $(\mathrm{HR})$ as the criterion measure. These validation experiments demonstrate that the $\mathrm{HR}$ and $\mathrm{VO}_{2}$ did not differ between cycling and running when compared at a given RPE ${ }^{38-41}$. The findings indicated that RPE can be used independently or conjunctively with $\mathrm{HR}$ and $\mathrm{VO}_{2}$ during exercise evaluations. In this context, RPE provides an alternative measure that is low cost and relies on minimal scale expertise to monitor exercise intensity. As opposed to physiological monitoring, perceived exertion monitoring requires no interruptions during the exercise training session or during physical activity participation.

Borg's scales of perceived exertion have been validated in a number of ambient environmental conditions and modes of activity. They have also stimulated the design of additional RPE scales ${ }^{2-4,44,45}$. These new scales not only rely on verbal descriptors but also on pictorial descriptors to provide visual "cognitive text" for perceptual responses ${ }^{2,15}$. The majority of these pictorial-verbal metrics are known as the OMNI Scales with various formats specific to children and adults. The OMNI Scales are appropriate for different modes of exercise such as walking/running, cycling, stepping, and resistance exercise. Each one of these scales has undergone experimental validation using the same physiological correlates that Borg used in developing the original RPE scales ${ }^{2-4,43-45}$. 


\subsubsection{Undifferentiated/Differentiated RPE}

RPE measurements can be undifferentiated for the overall body or can be anatomically differentiated to specific body regions. The differentiated RPE distinguishes between anatomically regionalized perceptual signals. Examples of these types of measurements are RPE for the overall body (RPE-O), legs (RPE-L), and chest (RPE-C) during aerobic exercises. During resistance exercise, RPE can be measured for the active muscle (RPE-AM). The differentiated RPE provides information particular to exercise prescription, and exertional dyspnea by targeting the level of exertion arising from a specified body region (i.e. chest, legs) involved in exercise. Additionally, the undifferentiated RPE is a good indicator of general exertion and often approximates the mathematical average of the differentiated RPE values ${ }^{2,15}$.

\subsection{PUBLIC HEALTH APPLICATIONS}

Many physical activity guidelines and recommendations follow a global approach to health where survival, instead of quality-adjusted life expectancy, is the primary concern. Typically, the minimum duration of activity is recommended for any given relative effort intensity ${ }^{42}$. As such,

Shephard ${ }^{42}$ clearly identified seven major future research directions. Of the seven, three are particularly important to the present investigation: 1) greater attention on age, gender, and fitness related differences in physical activity requirements, 2) a need to distinguish differences between relative and absolute intensity requirements, and 3) physical activity recommendations need to 
consider relative perceptual intensity ceilings ${ }^{42}$. The relative exercise intensity as measured by RPE can be used in exercise prescription, athletic training and rehabilitation 43, 44, 46, 54, 55 . Furthermore, a perceptual measure of relative physical activity intensity is not differentially influenced by age, gender, or race ${ }^{44,45,47-49}$. Applications of ratings of perceived exertion aside from self-reported RPE include exertional observation, session, and recall.

\subsubsection{Exertional Observation}

Exertional observation is a measurement tool that can be used by clinicians and exercise trainers to monitor progress throughout the course of the training session for a subject or client. A limited number of previous investigations have examined the validity and the application of exertional observation, mostly with adult populations ${ }^{51}$. Typically the observer estimates an RPE for the subject using standardized categories and keys such as sweating, breathing frequency, and difficulty of the exercise.

Exertional observation uses procedures found in the field of visual perception, physical activity assessment, and perceived exertion. The procedure includes an observation coding system that employs contextual categories and observation keys, and a pictorial-numerical RPE scale. In physical activity assessments, a partial interval sampling coding system is used to capture a "window of information" from which the behavioral pattern is recorded. The contextual categories are used as "signs and symptoms" relating to the individual (i.e. subject) and the environment in which that individual is placed and/or the exercise is performed. Contextual categories for the subject can be regionalized to six anatomical units (head/neck, right and left shoulders, trunk/back, right and left legs) that provide references for kinematic

evaluations of exertion ${ }^{2,51}$. The contextual categories for the environment represent physical 
locale, presence of an audience (i.e. spectators or laboratory investigators), and the facilities and equipment.

Each contextual category contains observation keys that "filter excess visual noise" and provide a "fine calibration" of the observed exercise perceptual response ${ }^{2}$. Typical observation keys include: breathing (frequency, light/heavy), body movements (lean, sway, hunched shoulders, vibration/shock), appearance (facial expressions, skin redness, sweating), muscles (trembling, strain, static/dynamic force), and sounds from the subject (groans/moans) ${ }^{2}$. Additionally, the observers are trained to minimize subject reactivity ${ }^{2,}{ }^{51}$. In a laboratory experiment, the observer sits at approximately a $45^{\circ}$ left-frontal angle to the subject. This position insures that the observer will not be able to detect the subject's perceived exertion response.

\subsubsection{Exertional Session}

Recently, session RPE has been gaining popularity in research studies as a measure of the total exertional perception for the entire exercise period (session). Session RPE is defined as the ratings of perceived exertion for the entire exercise session providing an overall quantitative measure of the effort experienced during the workout ${ }^{52-55}$. In conjunction with exertional observation, session RPE may allow clinicians and trainers to estimate the intensity at which their subjects or clients performed their exercise sessions. A differentiated session RPE can also be estimated for the arms, legs, chest, or the active muscles.

Foster et al. ${ }^{52-55}$ demonstrated that session RPE is a valid and reliable method to quantify various intensities of aerobic and resistance exercise. Moderate-to-strong correlations $(r=0.50$ to 
0.90) were reported between session RPE and the relative exercise intensity (i.e. \%HRR, $\left.\% \mathrm{VO}_{2 \max }, \% 1-\mathrm{RM}\right)$.

\subsubsection{Exertional Recall}

Ratings of perceived exertion, like physical activity or exercise intensity, can be assessed as a recall measurement. The methods for assessing recall RPE are similar to those employed in physical activity and exercise intensity questionnaires. An exertional recall questionnaire asks an individual to recall the level of exertion they felt during physical activity that occurred previously during a specific time period. As mentioned above, the most common questionnaires asked the participants to recall physical activity $24-72$ hours, 7 days, or up to a year previously.

In 2001, Serrano et al. ${ }^{56}$ studied the relation between perceived exertion and blood lactate concentrations in a Judo sports competition in a venue outside of a clinical or laboratory setting. The investigation used "Recall of Perceived Exertion" procedures that asked the competitors to retrospectively estimate their RPE ten minutes after their last Judo fight and then again at thirty minutes after the completion of the total competition. The competitors had been previously oriented to the RPE scales (Borg 6-20 and Borg CR-10) in training situations and were aware of the scaling procedure that they would be asked to use at the end of an individual fight and the total competition.

It was found that recall RPE for the last Judo fight (post-10 minutes) was not significantly correlated with blood lactate concentrations for the last fight. On the other hand, the recall RPE for the total competition (post-30 minutes) showed a positive and significant Spearman correlation with maximum blood lactate concentration $(r=0.63)$ and the change in blood lactate concentrations over the course of the competition $(r=0.64)$. It is possible that the 
small sample size or the short mean fight length (4.5 minutes; Standard Error Mean $=.5)$ was not sufficient to induce the physiological or perceptual response necessary to demonstrate a correlation between the recall RPE and lactate response for the total competition. The recall RPE for the total competition demonstrated a promising trend for the use of this measure during sports competitions. However, the short recall time interval employed by Serrano et al. ${ }^{56}$ would not be applicable in public health settings.

One of the most important investigations to establish the importance of relative intensity measures determined using a physical activity questionnaire was conducted by Lee at al. ${ }^{1}$ Their investigation examined the association between the relative intensity of exercise and coronary heart disease (CHD) risk. Using a questionnaire distributed as part of the Harvard Alumni Health Study in 1988, male respondents were asked to report their activities and the rating of exertion associated with the activity. The absolute measures of exercise intensity were determined by asking the respondents to report their daily walking, stair climbing, and sports or recreational activities in the past week (7-days). The frequency and duration for each activity was compared to normative data to establish an estimated MET value for that activity ${ }^{1}$. The Borg CR-10 scale was used to assess RPE. The questionnaire asked, "When you are exercising in your usual fashion, how would you rate your level of exertion (degree of effort)?" This measure was taken as the relative exercise intensity.

There was a strong inverse relation between the relative intensity of exercise as measured by RPE and coronary heart disease risk among older men ${ }^{1}$. Additionally, it was found that the absolute measures of physical activity intensity (i.e. MET levels) were not related to coronary heart disease risk. 
Lee et al. ${ }^{1}$ findings support the use of relative measures of exercise intensity in the form of RPE to establish a relation between physical activity and coronary heart disease risk in older individuals. In order to better understand the influence of relative measures of exercise intensity, we can look at the differences between the absolute and relative measures found by Lee et al ${ }^{1}$. On the absolute intensity scale (i.e. estimated METs), the relative risk of coronary heart disease for men who participated in moderate intensity sports or recreational activities $(R R=.98)$ experienced similar rates of coronary heart disease as those men who participated in light intensity physical activities $(\mathrm{RR}=1.00)$. However, when BMI, hypertension, hypercholesterolemia, and diabetes mellitus were statistically controlled (in addition to age, smoking, alcohol consumption, and diet), the energy expenditure across all activities and intensities became attenuated and lost statistical significance ${ }^{1}$.

In contrast to the absolute measures of exercise intensity, the relative intensity as measured by recall RPE demonstrated that, when compared to those men that rated their exercise as "weak" or less intense, the relative risk of CHD for those men that participated in "moderate", "somewhat strong", and "strong" was 19\%, 38\%, and 40\% lower, respectively ${ }^{1}$. When additional variables (i.e. BMI, hypertension) were statistically controlled the relative measures were attenuated but still remained statistically significant. These findings demonstrate the inverse association between relative intensity of physical activity and CHD risk, even among men that did not satisfy current physical activity recommendations ${ }^{1}$. The differences between the absolute and relative measures of physical activity intensity suggest that 1) public health recommendations need to be tailored to the individual and that global recommendations are not appropriate for all demographics, and 2) perceptually based relative measures of physical activity 
intensity may yield more meaningful information regarding the role of regular physical activity participation in promoting cardiovascular health.

\subsubsection{Exercise Modes}

Physical activity is defined as the general movement of the body whereas exercise is considered organized body movement directed towards health and fitness ${ }^{57}$. Ratings of perceived exertion have been validated for a wide variety of exercise modes (i.e. walking/running, stationary

cycling, step-exercise, and resistance exercise) ${ }^{2-4}$. Additionally, recall questionnaires using absolute measures of exercise intensity have also been validated for a wide variety of exercise and physical activities. The typical individual, however, often employs a mix of exercise modes and associated metabolic pathways. Furthermore, a balanced training program involving cardiovascular, endurance, and strength activities is recommended to promote health-related fitness. Moreover, certain types of sports training are targeted at both aerobic and anaerobic metabolic pathways such as soccer, lacrosse, football, and basketball.

Mixed mode exercises are often associated with typical physical activity patterns in the general population and in sports-defined exercises. Health-fitness conditioning, leisure time activities, and competitive athletic training can involve multiple modes that activate both aerobic and anaerobic energy pathways. As such, an exertional recall questionnaire must be validated for multi-modal exercises in order to measure the relative intensity of the diversified activities that comprise a typical health-fitness conditioning program. 


\subsection{TAKING THE NEXT STEP}

It was proposed that an estimate of the relative intensity as a component in physical activity questionnaires would be helpful in assessing the level of disease risk consequent to physical activity participation. RPE provides an estimate of the relative exercise intensity. As such, RPE provides an alternative to the use of estimated absolute intensity measures in physical activity questionnaires.

Evidence from Serrano et al. ${ }^{56}$ and Lee et al. ${ }^{1}$ suggests that a recall RPE component of a physical activity questionnaire would provide an important perceptual assessment of the relative intensity of physical activity. Serrano et al. ${ }^{56}$ demonstrated that the correlation between recall RPE and blood lactate concentration was strong. In addition, Lee et al. ${ }^{1}$ indicated that relative intensity measures of activity yield valuable information regarding health risks. These convergent lines of investigation suggest that a standardized 7-day RPE recall procedure could be used as a component of a physical activity questionnaire. The inclusion of an exertional recall to measure the relative intensity of the exercise or physical activity can facilitate the development of public health agendas and recommendations. 


\subsection{METHODOLOGY}

\subsection{SUBJECTS}

Twenty-two female and eighteen male adults $(\mathrm{N}=40)$ aged between 18 and 35 years of age were used as subjects in this investigation. These subjects were clinically healthy, recreationally active and had normal body weight. The characteristics of the female and male subjects were, respectively (mean $\pm \mathrm{SD}$ ); age $21.5 \pm 3.1$ and $20.6 \pm 2.1 \mathrm{yr}$, and peak oxygen consumption $36.4 \pm$ 6.6 and $44.7 \pm 9.5 \mathrm{~mL} \cdot \mathrm{kg}^{-1} \cdot \mathrm{min}^{-1}$ (Table 4). The procedural definition of recreationally active was participation in aerobic and/or resistance exercise 2-3 days a week not associated with collegiate or professional sport participation for 30-60 minutes per workout. The "true" definition of recreationally active was participating in $4 \pm 1.1$ days a week for $74 \pm 50.3$ minutes per workout.

Table 4. Descriptive Characteristics of the Sample. Mean \pm SD

\begin{tabular}{lccc} 
& $\begin{array}{c}\text { Total } \\
(\mathrm{N}=\mathbf{4 0})\end{array}$ & $\begin{array}{c}\text { Female } \\
(\mathbf{N}=\mathbf{2 2})\end{array}$ & $\begin{array}{c}\text { Male } \\
(\mathbf{N}=\mathbf{1 8})\end{array}$ \\
\hline Age $(\mathrm{yrs})$ & $21.1 \pm 2.7$ & $21.5 \pm 3.1$ & $20.6 \pm 2.1$ \\
Height $(\mathrm{cm})$ & $171.5 \pm 10.3$ & $165.0 \pm 7.2$ & $179.4 \pm 7.6$ \\
Weight $(\mathrm{kg})$ & $70.7 \pm 14.8$ & $62.3 \pm 10.3$ & $80.9 \pm 13.0$ \\
VO $_{\text {2peak }}(\mathrm{ml} / \mathrm{kg} / \mathrm{min})$ & $40.2 \pm 9.0$ & $36.4 \pm 6.6$ & $44.7 \pm 9.5$ \\
Bicep 1-RM $(\mathrm{kg})$ & $13.2 \pm 5.6$ & $8.9 \pm 2.0$ & $18.4 \pm 3.8$ \\
Body Composition (\% Fat) & $17.6 \pm 8.0$ & $21.6 \pm 5.4$ & $12.4 \pm 8.0$ \\
\hline
\end{tabular}

All subjects had no, or limited, prior experience with ratings of perceived exertion. Screening of the subjects began with the question: "Have you been involved in any exercise 
research in Tress Hall?" A potential subject was excluded from participation if the answer was yes. Subjects were recruited from physical education classes and by recruitment flyers (Appendix A). At the time of recruitment and prior to exercise testing, potential subjects were screened for pre-existing conditions that would place them in the "high risk" stratification for non-physician supervised exercise testing according to the American College of Sports Medicine 10, 57 . These individuals were ineligible to participate. Individuals with orthopedic, cardiovascular and/or metabolic complications (i.e. coronary artery disease, prior myocardial infarction, peripheral vascular disease, chronic obstructive pulmonary disease and diabetes mellitus) or who are pregnant were excluded from participation. In addition, the Physical Activity Readiness Questionnaire (PAR-Q) was administered before initiating exercise testing (Appendix A). A potential subject was excluded from participation if they answered yes to any of the PAR-Q questions.

\subsection{EXPERIMENTAL DESIGN}

This investigation used a perceptual estimation, cross-sectional design consisting of (a) one Fitness Assessment Session, (b) one Circuit Exercise Session, and (c) one Recall-Criterion Session. Each subject undertook the three sessions presented in the following sequence: Fitness Assessment, Circuit Exercise, and Recall-Criterion. Each of the three sessions were separated by 7 days.

Aerobic fitness and muscle strength was measured during the Fitness Assessment Session. The Circuit Exercise Session and the Recall-Criterion Session implemented a circuit training format consisting of four stations: Cycling Station 1 (C1), Resistance Station 1 (R1), 
Cycling Station 2 (C2), and Resistance Station 2 (R2). The starting station for each subject was counterbalanced such that a subject began either at a cycling station or a resistance station. The intensities for each station were also counterbalanced throughout the protocol (Figure 2). For example, if a subject was counterbalanced to start at $\mathrm{C} 1$, the progression was: $\mathrm{C} 1 \rightarrow \mathrm{R} 1 \rightarrow \mathrm{C} 2 \rightarrow \mathrm{R} 2$ or $\mathrm{C} 1 \rightarrow \mathrm{R} 2 \rightarrow \mathrm{C} 2 \rightarrow \mathrm{R} 1$. If they were counterbalanced to start at $\mathrm{R} 2$, the progression was: $\mathrm{R} 2 \rightarrow \mathrm{C} 2 \rightarrow \mathrm{R} 1 \rightarrow \mathrm{C} 1$ or $\mathrm{R} 2 \rightarrow \mathrm{C} 1 \rightarrow \mathrm{R} 1 \rightarrow \mathrm{C} 2$.

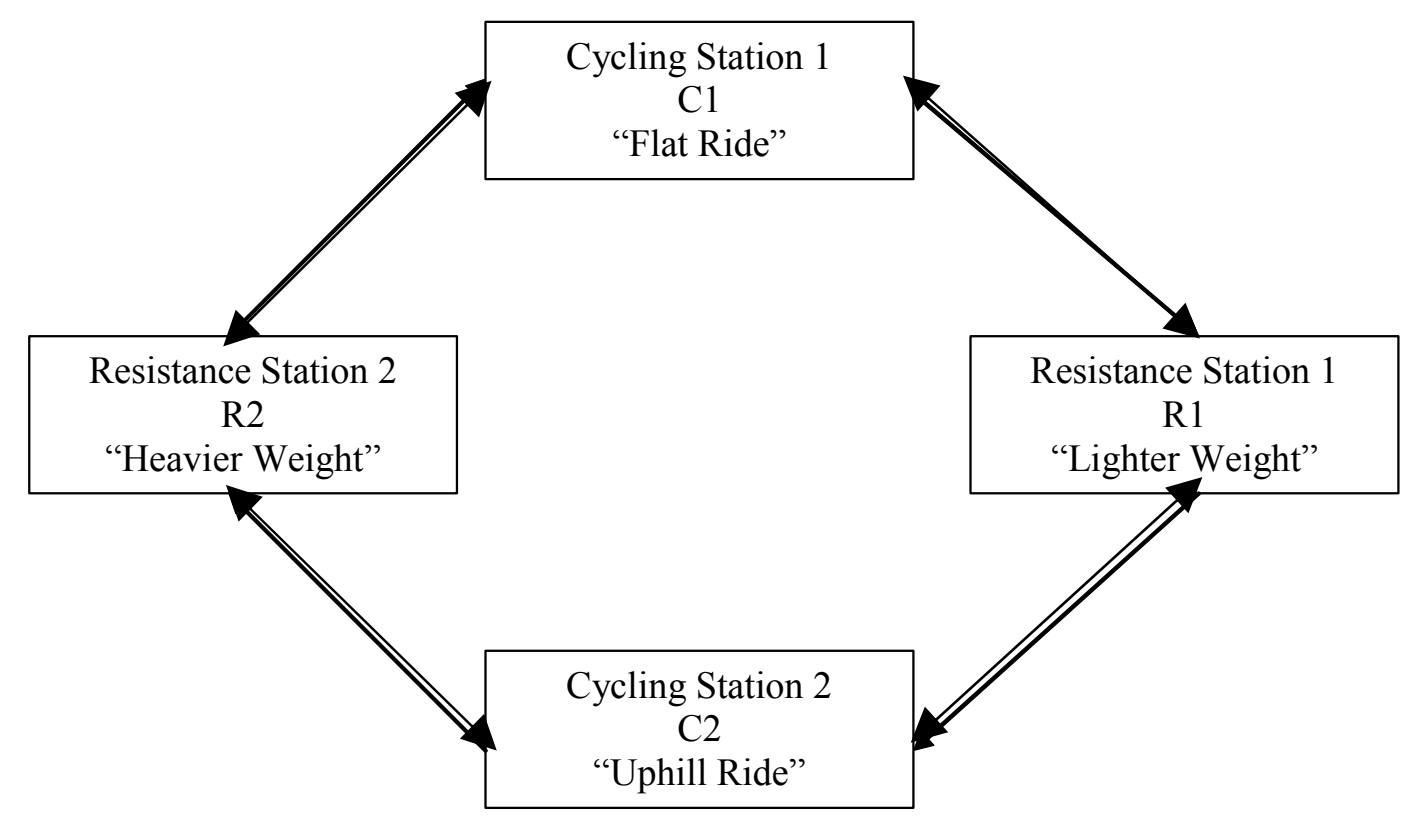

Figure 2. Circuit Training Program Flow-Chart

Within each mode of exercise, the intensity differed between the first and second stations. During the Circuit Exercise Session, the subjects did not estimate their RPE. Immediately prior to beginning the Recall-Criterion Session subjects were asked to recall their RPE experienced during the Circuit Exercise Session performed 7-days previously. The Recall RPE was determined using the 7-day exertional recall questionnaire. Subsequent to filling out the questionnaire, the subjects performed the exercise circuit with RPE actually measured. 


\subsection{EXERCISE PROTOCOLS}

\subsubsection{Fitness Assessment Session}

The Fitness Assessment Session was used to determine the peak aerobic power and bicep curl 1RM of the subjects. Descriptive characteristics were also recorded which included body height $(\mathrm{cm})$, body mass $(\mathrm{kg})$, and body fat $(\%)$. Body height was determined using a Detect-Medic Scale with an attached stadiometer (Detecto Scales, Inc., Brooklyn, NY). Body mass and fat was measured using a Tanita Bioelectrical Impedance Analysis Scale (Tanita Body Composition Analysis, Arlington Heights, IL). Arm dominance was determined by asking the subject to write their name on a piece of paper. The hand that was used to write their name was their dominant arm. The seat height on the Monark cycle ergometer was determined using American College of Sports Medicine (ACSM) Guidelines ${ }^{57}$. These guidelines stipulate that the subject should be positioned on the Monark cycle ergometer in an upright posture with a $5^{\circ}$ bend (flexion) in the knee at the maximal seated leg extension and hands placed in the proper position on the handlebars.

\subsubsection{Aerobic Fitness}

Peak aerobic power was assessed using a Modified Astrand cycle ergometer test (Appendix E). RPE was not assessed. The warm-up power output for both women and men was 50W and 2 minutes in duration. An electronic metronome was set to 100 beats per minute (50 pedal revolutions per minute) to signal the pedaling cadence. For men, the test power output began at $100 \mathrm{~W}$ and increased in $50 \mathrm{~W}$ increments every 3 minutes. For women, the test power output began at $75 \mathrm{~W}$ and increased in $25 \mathrm{~W}$ increments every 3 minutes. The test terminated when the subject was unable to maintain the designated pedal rate for 15 consecutive seconds or when the 
subject signaled that they were unable to continue due to fatigue, pain, injury or for any other reason. The measured peak oxygen consumption $\left(\mathrm{VO}_{2}\right.$ Peak $)$ was used to determine the aerobic fitness levels of each female and male subject. The $\mathrm{VO}_{2}$ Peak was also used to calculate the relative metabolic intensities $\left(\% \mathrm{VO}_{2 \text { Peak }}\right)$ for the cycling exercises that were undertaken in the Circuit Exercise and Recall-Criterion Sessions. These relative intensities were used to establish the metabolic range within which the validity of the exertional (cycle) recall questionnaire could be generalized. Oxygen consumption $\left(\mathrm{VO}_{2}\right.$; liters per minute; STPD) was measured using an open-circuit respiratory-metabolic system (True Max 2400, Parvo Medics, Salt Lake City, UT) during each minute of the cycle exercise protocol. Heart rate was measured from 45 to 60 seconds of each minute of the cycle exercise protocol. $\mathrm{VO}_{2 \text { peak }}$ was established when the subject was no longer able to perform the peak test due to fatigue. The termination coincided with a $\mathrm{VO}_{2}$ steady state, a RER $\geq 1.2$, and a maximal exercise heart rate $\pm 5 \mathrm{bpm}$ of age-predicted maximal heart rate.

\subsubsection{Biceps Muscle Strength}

Biceps strength was assessed using a 1-RM procedure described by Gearhart et al. ${ }^{59}$. RPE was not assessed. Using this procedure, the subjects were instructed to select a weight they feel they can lift only one time. The resistance was titrated until the subject reports he/she was near their 1-RM. This established a baseline for determining the 1-RM. If the initial baseline resistance was lifted successfully, 5 pounds for men and 2.5 pounds for women were added and the lift was repeated. The process continued until the subject was unable to complete the lift. Subjects rested for 2 minutes between lifts. The maximum weight successfully lifted through the complete range of motion was taken as the biceps curl 1-RM. If subjects were unable to successfully lift the initial baseline resistance, then 5 pounds was subtracted from the original 
weight. This process continued until it was not possible to successfully complete a biceps curl through the complete range of motion. The last weight successfully completed was taken as the biceps curl 1-RM. Following a 2 minute rest period, subjects were asked to repeat the maximum weight (plus one plate mate) they last successfully completed to verify the 1-RM.

\subsubsection{Circuit Exercise Session}

At the outset of the Circuit Exercise Session, the experiment was explained to the subjects. The purpose of the experiment was presented as a test to see if individuals were able to recall how hard they worked out the week before. Additionally, the format of the circuit stations was explained. No formal warm-up or cool-down sessions was incorporated in the circuit.

\subsubsection{Recovery}

A 30-60 second recovery period was placed between the stationary cycle and bicep curl resistance exercise stations, i.e. the time required for the subject to move from station to station and to position the respiratory-metabolic head bracket and mouthpiece on the subject.

\subsubsection{Cycling Exercise}

Power outputs of $50 \%$ and $75 \% \mathrm{VO}_{2 \text { Peak, }}$, as determined from the Fitness Assessment Session, was set by the investigator at the beginning of the "flat ride" and "uphill ride" cycling station; respectively. The power outputs elicited actual mean oxygen consumptions for the "flat" cycle bout at $62 \% \mathrm{VO}_{2 \text { peak }}$ and the "uphill" cycle bout at $87 \% \mathrm{VO}_{2 \text { peak. }}$. Subjects exercised on the Monark cycle ergometer for 5 minutes at a pedal rate of 50 revolutions per minute as signaled by an electronic metronome. 
During the cycling exercises, placards were shown before and after the exercise to indicate to the subject whether they are cycling on a flat surface or uphill. These same terms were used to distinguish the exercise intensities in the recall questionnaire. Oxygen consumption $\left(\mathrm{VO}_{2}\right.$; liters per minute; $\left.\mathrm{STPD}\right)$ was measured using an open-circuit respiratory-metabolic system (True Max 2400, Parvo Medics, Salt Lake City, UT) every 15 seconds of the cycle exercise. Heart rate (bpm) was measured with a Polar Monitoring System (Woodbury, NJ) from 45-60 seconds of each minute of exercise.

\subsubsection{Resistance Exercise - Biceps Curl}

Resistances of $60-70 \% 1-\mathrm{RM}$ and $90 \% 1$ 1-RM for the biceps curl exercises, as determined from the Fitness Assessment Session, were performed by the dominant arm for the "lighter weight" and the "heavier weight", respectively. The actual resistances for the "light" bicep curl bout was at $63 \% 1-\mathrm{RM}$ and the "uphill" bicep curl bout was at $86 \% 1-\mathrm{RM}$. Placards were shown before and after the exercise to indicate to the subject whether they are lifting a "lighter weight" or a "heavier weight". These terms were used to distinguish the intensities for the recall questionnaire. One set of 12 repetitions was performed for the "lighter weight" and one set of 6 repetitions was performed for the "heavier weight." Magnetic Plate Mates (Bodytrend, Carpenteria, CA) was used to adjust the biceps curl weight to the nearest .57 kilograms. The subject was seated on a flat exercise bench (Nautilus Fitness Products, Boulder, CO) having adjustable seat height and back support. Both feet were completely in contact with the floor, the knees and hips positioned in $45^{\circ}$ flexion and the back secure against the vertical back support. The inactive arm was maintained in $180^{\circ}$ extension throughout the exercise. The exercises begin with the barbell positioned at the closest point to the floor. At the end of the concentric phase, the elbow of the active arm will be in maximum flexion. During the eccentric phase, the barbell 
was lowered until the elbow is again in $180^{\circ}$ extension marking the completion of one full repetition. The concentric-eccentric muscle action of the biceps curl was performed in a vertical plane perpendicular to the horizontal plane of the shoulders. The concentric and eccentric phases were completed in 2 seconds with this pace signaled by an electronic metronome. A set is finished when the required repetitions for that exercise are completed. Incomplete repetitions were disregarded.

\subsubsection{Recall-Criterion Session}

The Recall-Criterion Session took place 7 days after the Circuit Exercise Session. During this session, the subject first completed the RPE Recall Questionnaire for the cycle and resistance exercises performed 7-days previously. Immediately following administration of the questionnaire, the procedure to estimate RPE was explained including a definition, scaling instructions, and scale anchoring procedures. Once the RPE orientation was completed, the subject performed the same exercise circuit as during their previous Circuit Exercise Session. However, during the Recall-Criterion Session, RPE was directly measured at regular intervals for each exercise throughout the circuit program. In the case of a failed set for the resistance exercise, RPE was determined immediately after termination.

\subsubsection{Recall Questionnaire}

Subjects were given two mode specific recall questionnaires and one circuit session questionnaire. These were used to recall their RPE experienced during the circuit exercise session performed 7-days earlier. The first questionnaire in the recall packet asked the subjects to rate their RPE-Session for the entire exercise session and to state which form of exercise most 
influenced their full session values. The subjects then received questionnaires corresponding to their counterbalanced progression sequence for the exercise modes (i.e. a subject who started at the stationary cycle station was given the cycle recall questionnaire first followed by the resistance exercise recall questionnaire). The cycle recall questionnaire (Appendix C) used the adult format of the OMNI-Cycle Scale (Appendix B) that included a brief set of scaling instructions. These instructions state: "Think about the exercise that you performed on the stationary cycle in the circuit program one week (7 days) ago. Please use this picture scale to rate the level of effort, strain, discomfort and/or fatigue that you felt during the cycling exercise. Using numbers from the above effort scale: Rate your effort during the flat cycle ride (RPEOverall, RPE-Legs, and RPE-Chest). Rate your effort during the uphill cycle ride (RPE-Overall, RPE-Legs, and RPE-Chest).” The resistance recall questionnaire (Appendix C) used the adult format of the OMNI-Resistance Scale (Appendix B) with accompanying instructions. The instructions for the resistance recall questionnaire were as follows: "Think about the resistance/lifting exercise that you performed during the circuit program one week ( 7 days) ago. Please use this picture scale to rate the level of effort, strain, discomfort and/or fatigue that you felt during the bicep curl exercise. Using numbers from the above effort scale: Rate your effort during the "lighter weight" resistance station (RPE-Overall, RPE-Arm). Rate your effort during the "heavier weight" resistance station (RPE-Overall, RPE-Arm). ${ }^{58}$

\subsubsection{Ratings of Perceived Exertion - Exercise Measurement}

Subjects were asked to rate their perceived exertion experienced during the Recall-Criterion Session. Prior to the start of the exercises, the subjects were oriented to the OMNI-Cycle and OMNI-Resistance Scales (Appendix B) using standardized instructions and anchoring procedures (Appendix D). These instructions were short, practical, and designed for the 
particular exercise mode that the subject will perform. The instructions satisfied the physiological-perceptual linkage presented in the Effort Continua Model. These instructions identified the lowest verbal, pictorial, and numerical cue on the scale representing very low exercise intensity. The instructions then identified the highest verbal, pictorial, and numerical cue on the scale representing maximal exercise. In order to facilitate understanding of the OMNI-RPE scale, memory anchoring procedures were used to reinforce the instructions. For aerobic exercise, the subject was asked to think about the effort he or she felt performing a low exercise intensity (i.e. resting, easy-walking) and to assign the lowest numerical rating on the scale equivalent to that feeling. Additionally, the subject was asked to remember the effort he or she felt during maximal or high intensity exercise (i.e. walking/running up a steep hill) and to assign the highest numerical rating of the scale equivalent to that feeling ${ }^{2}$. For resistance exercise, the subject was asked to think about the effort he or she felt lifting a very light weight (i.e. picking up a shirt, a water bottle) and to assign the lowest numerical rating on the scale equivalent to that feeling. Additionally, the subject was asked to remember the effort he or she felt lifting a heavy weight (i.e. a television, couch) and to assign the highest numerical rating on the scale equivalent to that feeling.

For the cycling stations, three separate RPE measurements were taken every 45 to 60 seconds of each minute of exercise. The adult format of the OMNI-Cycle Scale was used. An undifferentiated rating was measured for the overall body (RPE-O) and a differentiated rating was measured for the peripheral perceptions in the legs (RPE-L) and breathing perceptions in the chest (RPE-C). For the "lighter weight" biceps curl station, two separate RPE measurements were taken during the eccentric phase of every six repetitions. For the "heavier weight" biceps curl station, two separate RPE measurements were taken during the eccentric phase of ever three 
repetitions. The adult format of the OMNI-Resistance Scale was used. An undifferentiated rating was measured for the overall body (RPE-O) and a differentiated rating was measured for the peripheral perceptions in the arm (RPE-A).

\subsection{DATA ANALYSIS}

Descriptive data for anthropometric, perceptual and physiological variables were calculated as mean \pm standard deviation (SD). RPE responses for the circuit program were statistically examined using a two factor [Procedure (Recall vs. Criterion) $\mathrm{x}$ Sex] analysis of variance. For the cycle exercises, concurrent validation assessed the relation between oxygen consumption and RPE-O, -L, and $-\mathrm{C}$. RPE responses for the cycle exercise were statistically examined using a three factor [Procedure (Recall vs. Criterion) x Intensity (lower vs. higher) x Sex] analysis of variance. Separate analyses were conducted for RPE-O, -L, and -C. At the onset of this investigation, the concurrent validation of relation between total weight lifted for each station and both RPE-O and RPE-A for resistance exercise was planned. In retrospect, this investigation was unable to validate exertional recall using total weight lifted as a criterion variable because it did not differ between the two intensities for each individual. Furthermore, the number of repetitions at each intensity level did not differ between subjects participating in this investigation. RPE responses for the resistance exercise were statistically examined using a three factor [Procedure (Recall vs. Criterion) x Intensity (light vs. heavy) x Sex] analysis of variance. Separate analyses were conducted for RPE-O and -A. Significant main and sex interaction effects were probed with a Scheffe post-hoc procedure. Significant procedure and intensity interaction effects were probed with a two-factor analysis of variance. Statistical significance 
was accepted at the $\mathrm{p}<0.05$ level. Analysis of under-estimation and over-estimation of RPE responses was analyzed through Bland-Altman plots with a 95\% CI to identify possible grouping effects of sex. 


\subsection{RESULTS}

This investigation examined the validity of a recall questionnaire to measure the perception of physical exertion experienced previously during standardized bouts of stationary cycling and resistance exercise for separate groups of female and male adults. The investigation used a perceptual estimation, cross-sectional design consisting of: (a) one Fitness Assessment Session, (b) one Circuit Exercise Session, and (c) one Recall-Criterion Session. Each of the three sessions were separated by 7 days and presented in the following sequence: Fitness Assessment, Circuit Exercise, and Recall-Criterion.

\subsection{DESCRIPTIVE INFORMATION}

The means $( \pm \mathrm{SD})$ of the Recall and Criterion RPE responses for cycle and resistance exercise are presented separately for females (Figures 3 and 4 ) and for males (Figures 5 and 6). Appendix F lists these descriptive data in table format. 


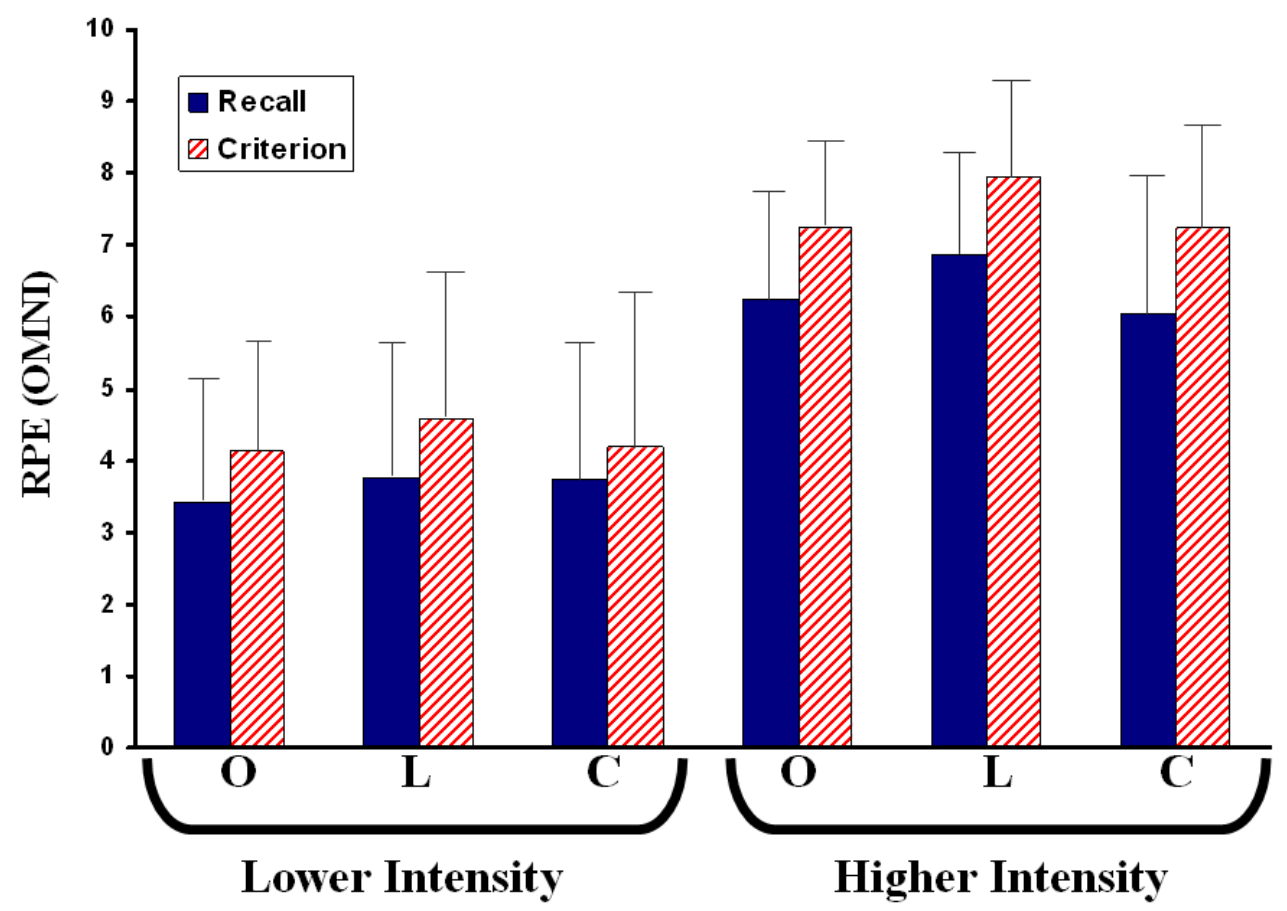

Figure 3. Mean ( \pm SD) Recall and Criterion RPE during Cycle Exercise for Females

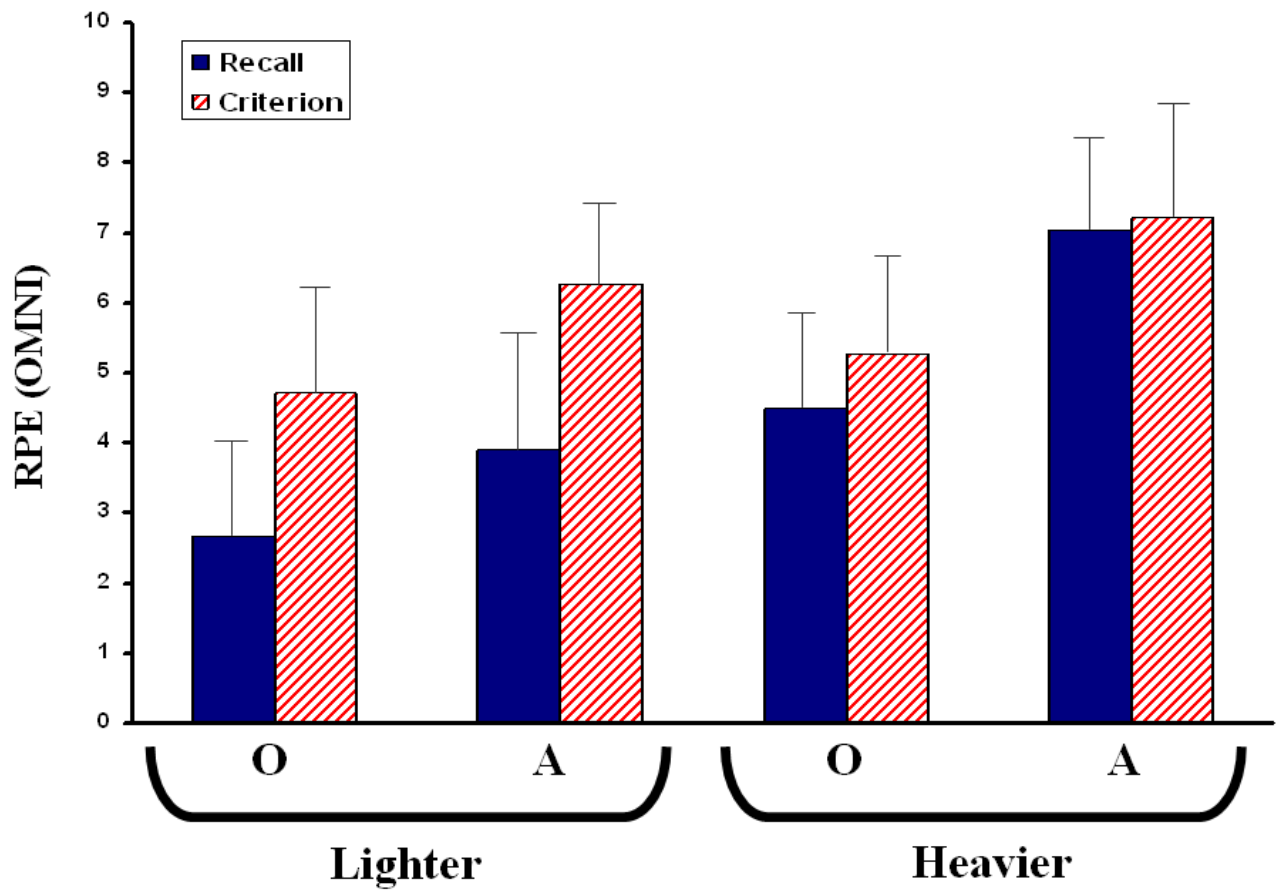

Figure 4. Mean ( \pm SD) Recall and Criterion RPE during Resistance Exercise for Females RPE: Rating of Perceived Exertion.

O: RPE - Overall Body, L: RPE - Leg, C: - RPE - Chest, A; RPE - Arm 


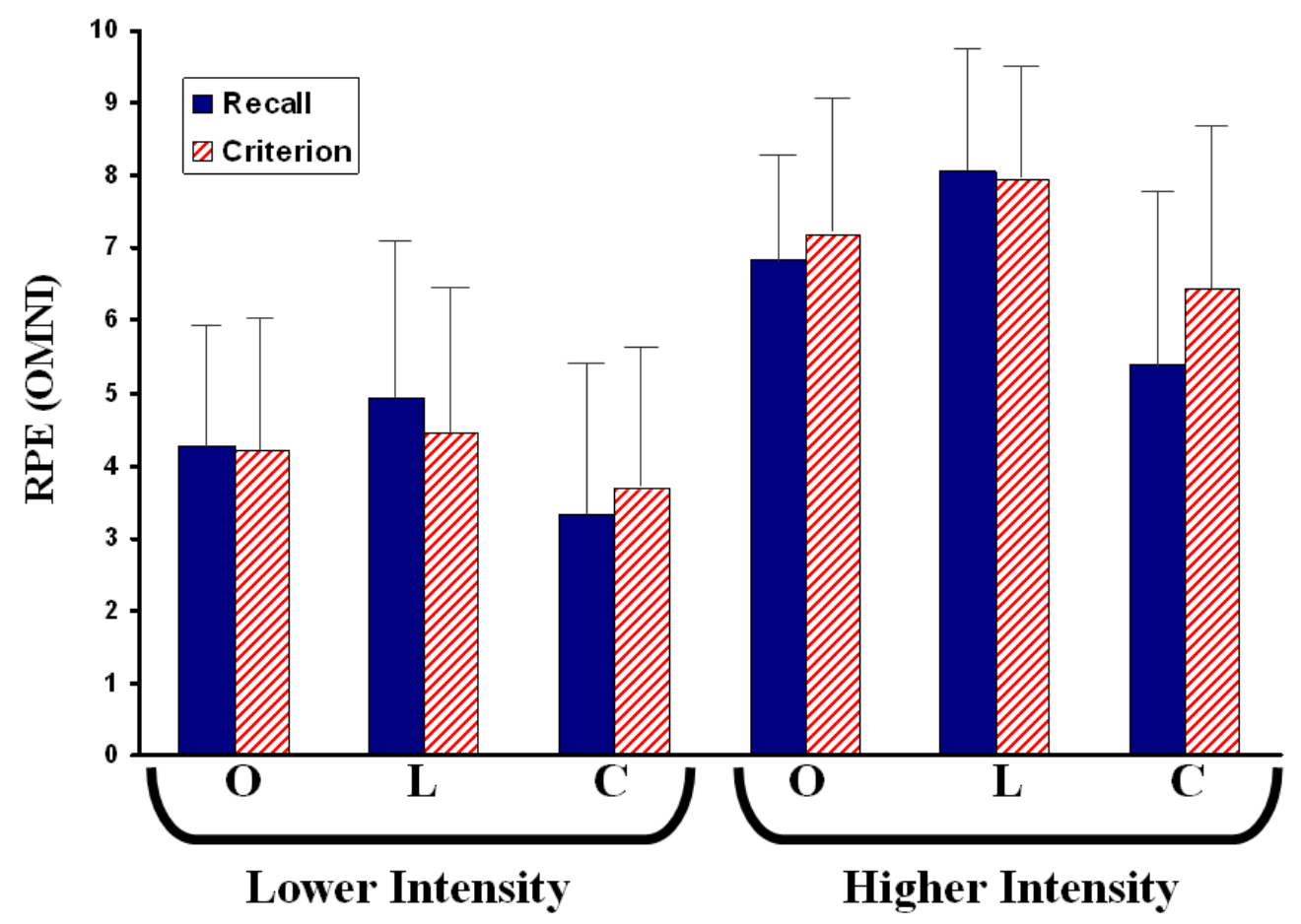

Figure 5. Mean $( \pm$ SD) Recall and Criterion RPE during Cycle Exercise for Males

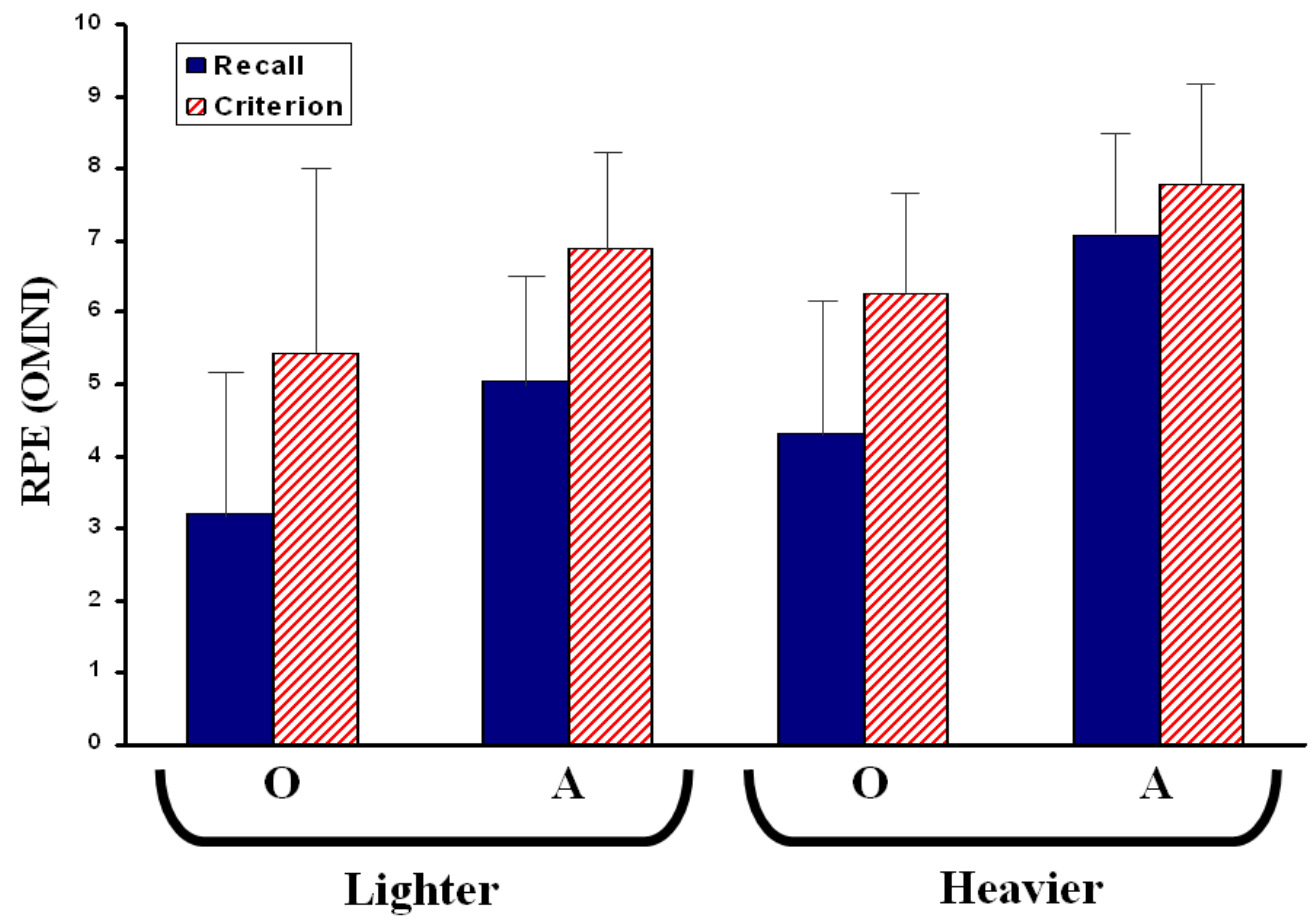

Figure 6. Mean ( \pm SD) Recall and Criterion RPE during Resistance Exercise for Males RPE: Rating of Perceived Exertion.

O: RPE - Overall Body, L: RPE - Leg, C: - RPE - Chest, A; RPE - Arm 


\subsection{EXERTIONAL RECALL}

\subsubsection{Cycling Exercise}

Construct validity was examined as the relation between Recall and Criterion RPE and calculated separately for the undifferentiated and differentiated ratings (Table 5). For cycle exercise bouts, significant moderate-to-strong correlations ( $r=0.427$ to 0.671$)$ were observed between Recall and Criterion RPE at both intensities.

Table 5. Pearson Correlations between Recall and Criterion Ratings of Perceived Exertion (RPE) for Cycle Exercise

\begin{tabular}{cccc}
\hline & \multicolumn{3}{c}{ RPE } \\
\cline { 2 - 4 } Intensity & Overall Body & Legs & Chest \\
\hline \multirow{2}{*}{ Lower } & $0.556^{* *}$ & $0.592^{* *}$ & $0.671^{* *}$ \\
Higher & $0.427^{* *}$ & $0.537^{* *}$ & $0.541^{* *}$ \\
& & & \\
\hline$* * \mathbf{p}<\mathbf{0 . 0 1}$ level (2-tailed). & &
\end{tabular}

Concurrent validation was examined by correlating $\mathrm{VO}_{2}$ with $\mathrm{RPE}-\mathrm{O}$, $-\mathrm{L}$, and $-\mathrm{C}$ for each cycling intensity. Weak-to-moderate correlations $(r=-0.005$ to 0.409$)$ were observed between the Recall RPE and $\mathrm{VO}_{2}$ (Table 6). Similarly, weak-to-moderate correlations $(\mathrm{r}=0.097$ to 0.440 ) were observed between the Criterion $\mathrm{RPE}$ and $\mathrm{VO}_{2}$ at each intensity. When the data from the two cycling intensities were combined in the analyses, moderate correlations $(\mathrm{r}=0.432$ to 0.633 ) were observed between Criterion RPE and $\mathrm{VO}_{2}$. Using combined data, lower-tomoderate correlations ( $\mathrm{r}=0.258$ to 0.569$)$ were observed between Recall RPE and $\mathrm{VO}_{2}(\mathrm{Table}$ $6)$. 
Table 6. Pearson Correlations between Oxygen Consumption and RPE

\begin{tabular}{lcccc}
\hline & & \multicolumn{3}{c}{ RPE } \\
\cline { 3 - 5 } Procedure & Intensity & Overall Body & Legs & Chest \\
\hline Criterion & Lower & $0.376^{*}$ & $0.440^{* *}$ & 0.225 \\
& Higher & $0.344^{*}$ & $0.433^{* *}$ & 0.097 \\
& Total & $0.571^{* *}$ & $0.633^{* *}$ & $0.432^{\star *}$ \\
Recall & Lower & 0.090 & 0.275 & 0.010 \\
& Higher & 0.241 & $0.409^{* *}$ & -0.005 \\
& Total & $0.457^{* *}$ & $0.569^{* *}$ & $0.258^{\star *}$ \\
\hline
\end{tabular}

$* * \mathbf{p}<0.01$ level (2-tailed).

$* \mathbf{p}<0.05$ level (2-tailed).

Rating of perceived exertion responses were examined with a three-factor [Procedure (Recall vs. Criterion) x Intensity (lower vs. higher) x Sex] analysis of variance. Separate analyses were conducted for the undifferentiated (RPE-O) and the differentiated (RPE-L and RPE-C) perceptual responses. Mauchly's test of sphericity was not significant for RPE-O, -L, and -C.

\subsubsection{Overall Body Responses}

The results of the analysis of variance for RPE-O are presented in Table 7. There was not a significant main effect of procedure and sex on RPE-O. As expected, there was a main effect of intensity on RPE-O. The significant intensity main effect indicated that RPE-O was greater $(\mathrm{p}<$ 0.001) during the higher than lower cycling intensity. Interaction effects of procedure $\mathrm{X}$ intensity, procedure $\mathrm{X}$ sex, intensity $\mathrm{X}$ sex, and procedure $\mathrm{X}$ intensity $\mathrm{X}$ sex were not significant for RPE-O. 
Table 7. Results of the Analysis of Variance for RPE-O during Cycle Exercise

\begin{tabular}{lcrcc}
\hline Main Effects & df & \multicolumn{1}{c}{$\mathbf{F}$} & $\boldsymbol{p}$ & $\boldsymbol{\eta}^{\mathbf{2}}$ \\
\hline Procedure (P) & 1 & 3.636 & 0.064 & 0.087 \\
Intensity (I) & 1 & 173.453 & $<0.001^{*}$ & 0.820 \\
Sex (S) & 1 & 0.603 & 0.442 & 0.016 \\
& & & & \\
Interaction Effects & & & & \\
\hline P x I & 1 & 1.223 & 0.276 & 0.031 \\
P x S & 1 & 5.200 & 0.176 & 0.048 \\
Ix S & 1 & 0.223 & 0.639 & 0.006 \\
P x Ix S & 1 & 0.038 & 0.847 & 0.001 \\
& & & & \\
Error & 38 & & & \\
\hline
\end{tabular}

* Statistically significant (2-tailed)

\subsubsection{Leg Responses}

The results of the analysis of variance for RPE-L are presented in Table 8 . There was not a significant main effect of procedure and sex on RPE-L. As expected, there was a main effect of intensity on RPE-L. The significant intensity main effect indicated that RPE-L was greater $(\mathrm{p}<$ 0.001) during the higher than lower cycling intensity. Interaction effects of procedure $\mathrm{X}$ intensity, intensity X sex, and procedure X intensity X sex were not significant for RPE-L. There was a significant interaction effect of procedure $\mathrm{X}$ sex (Appendix G.1). Post-hoc analysis indicated that Recall RPE-L was not significantly different between females and males at the lower cycling intensity, $p=0.096$. However, Recall RPE-L was significantly higher for males than females at the higher cycling intensity, $p=0.033$. The post-hoc analysis did not indicate difference in RPE-L between procedures. 
Table 8. Results of the Analysis of Variance for RPE-L during Cycle Exercise

\begin{tabular}{lcrcc}
\hline Main Effects & df & \multicolumn{1}{c}{$\mathbf{F}$} & \multicolumn{1}{c}{$\boldsymbol{p}$} & $\boldsymbol{\eta}^{\mathbf{2}}$ \\
\hline Procedure (P) & 1 & 1.968 & 0.169 & 0.049 \\
Intensity (I) & 1 & 227.953 & $<0.001^{*}$ & 0.857 \\
Sex (S) & 1 & 1.151 & 0.290 & 0.029 \\
& & & & \\
Interaction Effects & & & & \\
\hline P x I & 1 & 1.171 & 0.286 & 0.030 \\
P x S & 1 & 7.418 & $0.010^{*}$ & 0.163 \\
I X S & 1 & 0.033 & 0.857 & 0.001 \\
P x I x S & 1 & 0.036 & 0.850 & 0.001 \\
& & & & \\
Error & 38 & & & \\
\hline
\end{tabular}

* Statistically significant (2-tailed)

\subsubsection{Chest Responses}

The results of the analysis of variance for RPE-C are presented in Table 9. There was a significant main effect of procedure and intensity on RPE-C. There was not a significant main effect of sex on RPE-C between females and males. Interaction effects of procedure X sex, intensity $\mathrm{X}$ sex, and procedure $\mathrm{X}$ intensity $\mathrm{X}$ sex were not significant for RPE-C. There was a significant interaction effect of procedure X intensity (Appendix G.1). Post-hoc analysis indicated Recall RPE-C was not significantly different from the Criterion RPE-C for the lower cycling intensity, $F(1,39)=1.926, p=0.173$. However, Recall RPE-C was significantly lower than Criterion RPE-C for the higher cycling intensity, $F(1,39)=11.323, p=0.002$. The posthoc analyses did not indicate difference in RPE-C between procedures. 
Table 9. Results of the Analysis of Variance for RPE-C during Cycle Exercise

\begin{tabular}{lcrcc}
\hline Main Effects & df & \multicolumn{1}{c}{$\mathbf{F}$} & \multicolumn{1}{c}{$\boldsymbol{p}$} & $\boldsymbol{\eta}^{2}$ \\
\hline Procedure (P) & 1 & 7.273 & $0.010^{*}$ & 0.161 \\
Intensity (I) & 1 & 151.855 & $<0.001^{*}$ & 0.800 \\
Sex (S) & 1 & 0.988 & 0.326 & 0.025 \\
& & & & \\
Interaction Effects & & & & \\
\hline P x I & 1 & 5.900 & $0.020^{*}$ & 0.134 \\
P x S & 1 & 0.049 & 0.827 & 0.001 \\
I x S & 1 & 0.411 & 0.525 & 0.011 \\
P x I x S & 1 & $<0.001$ & 0.993 & $<0.001$ \\
& & & & \\
Error & 38 & & & \\
\hline * Statistically significant (2-tailed) & & &
\end{tabular}

* Statistically significant (2-tailed)

\subsubsection{Resistance Exercise}

Construct validity was examined as the relation between Recall and Criterion RPE and calculated separately for the undifferentiated and differentiated ratings (Table 10). Low but significant correlations were found between Recall and Criterion RPE-O at both resistance intensities. Recall and Criterion RPE-A were not correlated at either intensity.

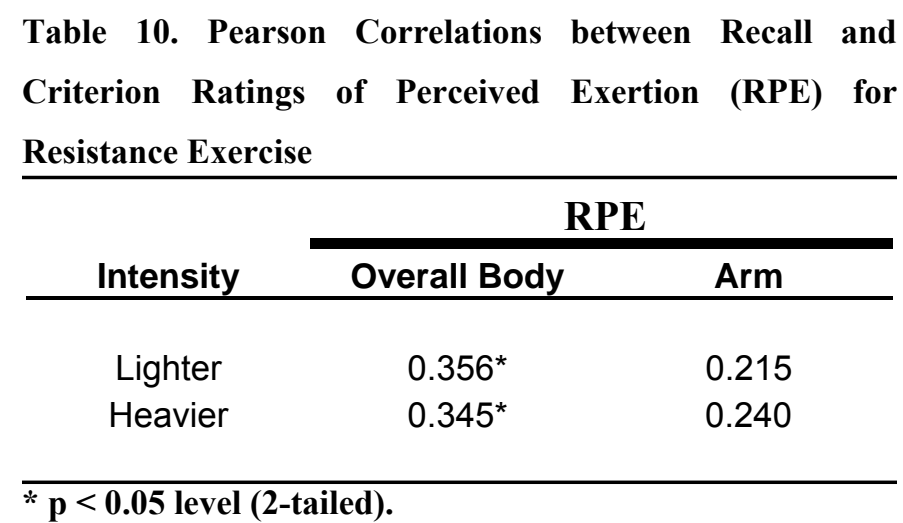

Ratings of perceived exertion were examined with a three-factor [Procedure (Recall vs. Criterion) x Intensity (light vs. heavy) x Sex] analysis of variance. Separate analyses were 
conducted for the undifferentiated (RPE-O) and the differentiated (RPE-A) perceptual responses. Mauchly’s test of sphericity was not significant for RPE-O, and-A.

\subsubsection{Arm Responses}

The results of the analysis of variance for RPE-A are presented in Table 11. There was a significant main effect of procedure and intensity on RPE-A. There was not a significant main effect of sex on RPE-A between females and males. Interaction effects of procedure X sex, intensity $\mathrm{X}$ sex, and procedure $\mathrm{X}$ intensity $\mathrm{X}$ sex were not significant for RPE-A. There was a significant interaction effect of procedure $\mathrm{X}$ intensity (Appendix G.2). Post-hoc analysis indicated that Recall RPE-A was not significantly different from Criterion RPE-A for the heavier resistance bout, $F(1,39)=2.016, p=0.164$. However, Recall RPE-A was significantly lower than Criterion RPE-A for the lighter resistance bout, $F(1,39)=40.867, p<0.001$. The post-hoc analyses did not indicate difference in RPE-A between procedures.

Table 11. Results of the Analysis of Variance for RPE-A during

Resistance Exercise

\begin{tabular}{lcrcr}
\hline Main Effects & df & \multicolumn{1}{c}{$\mathbf{F}$} & $\boldsymbol{p}$ & $\boldsymbol{\eta}^{\mathbf{2}}$ \\
\hline Procedure (P) & 1 & 20.398 & $<0.001^{*}$ & 0.349 \\
Intensity (I) & 1 & 84.227 & $<0.001^{*}$ & 0.689 \\
Sex (S) & 1 & 2.915 & 0.096 & 0.071 \\
& & & & \\
Interaction Effects & & & & \\
\hline P x I & 1 & 30.660 & $<0.001^{*}$ & 0.447 \\
P x S & 1 & $<0.001$ & 0.993 & $<0.001$ \\
I x S & 1 & 2.498 & 0.122 & 0.062 \\
P x I x S & 1 & 3.241 & 0.080 & 0.079 \\
& & & & \\
Error & 38 & & & \\
* Statistically significant (2-tailed) & & &
\end{tabular}

* Statistically significant (2-tailed) 


\subsubsection{Overall Body Responses}

The results of the analysis of variance for RPE-O are presented in Table 12. There was a significant main effect of procedure and intensity on RPE-O. There was not a significant main effect of sex on RPE-O between females and males. Interaction effects of procedure $\mathrm{X}$ sex, intensity $\mathrm{X}$ sex, and procedure $\mathrm{X}$ intensity $\mathrm{X}$ sex were not significant for RPE-O. There was a significant interaction effect of procedure $\mathrm{X}$ intensity (Appendix G.2). Post-hoc analysis indicated Recall RPE-O was significantly lower than Criterion RPE-O for the lighter resistance bout, $F(1,39)=46.845, p<0.001$. Recall RPE-O was significantly lower than Criterion RPE-O for the heavier resistance bout, $F(1,39)=16.644, p<0.001$. The post-hoc analyses did not indicate difference in RPE-O between procedures.

Table 12. Results of the Analysis of Variance for RPE-O during

Resistance Exercise

\begin{tabular}{lcrcc}
\hline Main Effects & df & \multicolumn{1}{c}{$\mathbf{F}$} & $\boldsymbol{p}$ & $\boldsymbol{\eta}^{\mathbf{2}}$ \\
\hline Procedure (P) & 1 & 43.311 & $<0.001^{*}$ & 0.533 \\
Intensity (I) & 1 & 51.326 & $<0.001^{*}$ & 0.800 \\
Sex (S) & 1 & 1.504 & 0.228 & 0.038 \\
& & & & \\
Interaction Effects & & & & \\
\hline P x I & 1 & 5.449 & $0.025^{*}$ & 0.125 \\
P x S & 1 & 1.614 & 0.212 & 0.041 \\
I x S & 1 & 0.486 & 0.490 & 0.013 \\
P x I x S & 1 & 2.244 & 0.142 & 0.056 \\
& & & & \\
Error & 38 & & & \\
* Statistically significant (2-tailed) & & &
\end{tabular}

\subsubsection{Circuit Program RPE-Session}

A two-factor [Procedure (Recall vs. Criterion) x Sex] analysis of variance examined differences in RPE-Session. There was a significant main effect of procedure on RPE-Session. This indicated that Recall RPE-Session was significantly higher than Criterion RPE-Session, $F(1,38)$ 
$=4.944, p=0.032$, partial $\eta^{2}=0.113$. There was not a significant main effect of sex on RPESession, $F(1,38)=.882, p=0.353$, partial $\eta^{2}=.023$. The interaction effect of procedure $\mathrm{X}$ sex was not significant for RPE-Session, $F(1,38)=.267, p=0.609, \eta^{2}=0.007$.

\subsection{ANALYSIS OF UNDER-ESTIMATION AND OVER-ESTIMATION}

Bland-Altman plots assessed the level of agreement in RPE between Recall and Criterion procedures. The Bland-Altman plots display differences in RPE between Recall and Criterion procedures for individual subjects. As such, these plots were used to determine under-estimation and over-estimation of the Recall RPE. The mean difference (or bias) is indicated by a solid line. The $95 \%$ confidence interval ( $\pm 2 \mathrm{SD}$; upper and lower limits of agreement) is indicated by dashed lines. Additionally, the range displayed on the y-axis represents the maximum possible RPE difference between the Recall and Criterion procedures. Responses were analyzed separately for RPE-O, -L, and -C for both intensities of cycle exercise (Figure 7) and RPE-O and -A for both intensities of resistance exercise (Figure 8).

\subsubsection{Limits of Agreement for Cycle Exercise}

Bland-Altman plots demonstrated a good inter-procedure agreement between Recall and Criterion RPEs for females and males during cycle exercise at both intensities. The mean difference indicated slight under-estimations of RPE-O, -L, and -C by the Recall procedure in comparison to the Criterion procedure. Follow-up frequency distributions for all cases ( 80 total cases $=40$ subjects $\times 2$ intensities) presented in the Bland-Altman plots are listed in Table 13. RPE was also converted to standardized scores and presented in Bland-Altman plots (see 
Appendix H.1). Standardized RPE scores were examined to allow comparison of data between different category rating scales. Effect sizes for males for RPE-O, $-\mathrm{L}$, and $-\mathrm{C}$ were $-0.06,0.12$, and -0.27 . Effect sizes for females were $-0.33,-0.36$, and -0.33 . The effect sizes for the combined data set of males and females were $-0.21,-0.15$, and -0.30 . All effect sizes were low. The frequency table lists the observed range of mean difference values between Recall and Criterion RPE-O, -L, and $-\mathrm{C}$ obtained from the three separate Bland-Altman plots. Furthermore, the majority of the mean difference values fell between a) -2 (an under-estimation of the Recall procedure) and 1 (an over-estimation of the Recall procedure) for both RPE-O and $-\mathrm{L}$; and b) -3 (an under-estimation of the Recall procedure) and 1 (an over-estimation of the Recall procedure) for RPE-C. 

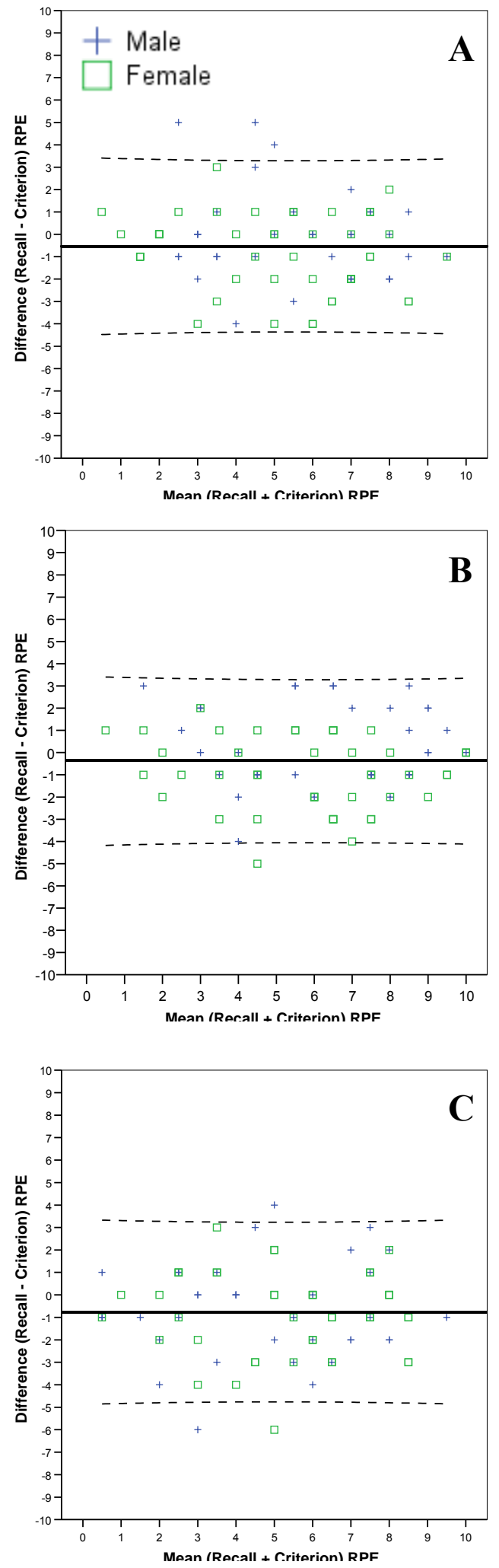

Figure 7. Bland-Altman Plots of (A) RPE-O, (B) RPE-L, and (C) RPE-C for Two Intensities of Cycling Exercise 
Table 13. Frequency Distribution of the RPE Differences between Recall and Criterion Procedures for Cycle Exercise*

\begin{tabular}{cccc}
\hline Difference & RPE-O & RPE-L & RPE_C \\
\hline-6 & 0 & 0 & 2 \\
-5 & 0 & 1 & 0 \\
-4 & 5 & 2 & 4 \\
-3 & 6 & 7 & 10 \\
-2 & 14 & 10 & 11 \\
-1 & 15 & 22 & 16 \\
0 & 19 & 12 & 16 \\
1 & 14 & 13 & 12 \\
2 & 2 & 7 & 5 \\
3 & 2 & 6 & 3 \\
4 & 1 & 0 & 1 \\
5 & 2 & 0 & 0 \\
\hline
\end{tabular}

* Frequencies based on Bland-Altman Plots in Figure 7.

\subsubsection{Limits of Agreement for Resistance Exercise}

Bland-Altman plots demonstrated a poor inter-procedure agreement between Recall and Criterion RPEs for females and males during resistance exercise (Figure 8). The mean difference values between procedures indicated comparatively greater under-estimations of RPE-O and -A by the Recall procedure than the Criterion procedure. Follow-up frequency distributions for all cases ( 80 total cases $=40$ subjects $\times 2$ intensities) in the Bland-Altman plots are listed in Table 14. RPE was also converted to standardized scores and presented in Bland-Altman plots (see Appendix H.2. Standardized RPE scores were examined to allow comparison of data between different category rating scales. Effect sizes for males for RPE-O, and -A were -1.08 and -1.03. Effect sizes for females were -0.97 and -0.71 . The effect sizes for the combined data set of males and females were -1.01 and -0.83 . All effect sizes were considered large and consistent with the ANOVA results. The frequency table lists the observed range of mean difference values between Recall and Criterion RPE-O and -A for the two Bland-Altman plots. The majority of the mean difference values fell between a) -4 (an under-estimation of the Recall procedure) and 0 (no bias 
in the procedure) for RPE-O; and b) -3 (an under-estimation of the Recall procedure) and 1 (an over-estimation of the Recall procedure) for RPE-A.
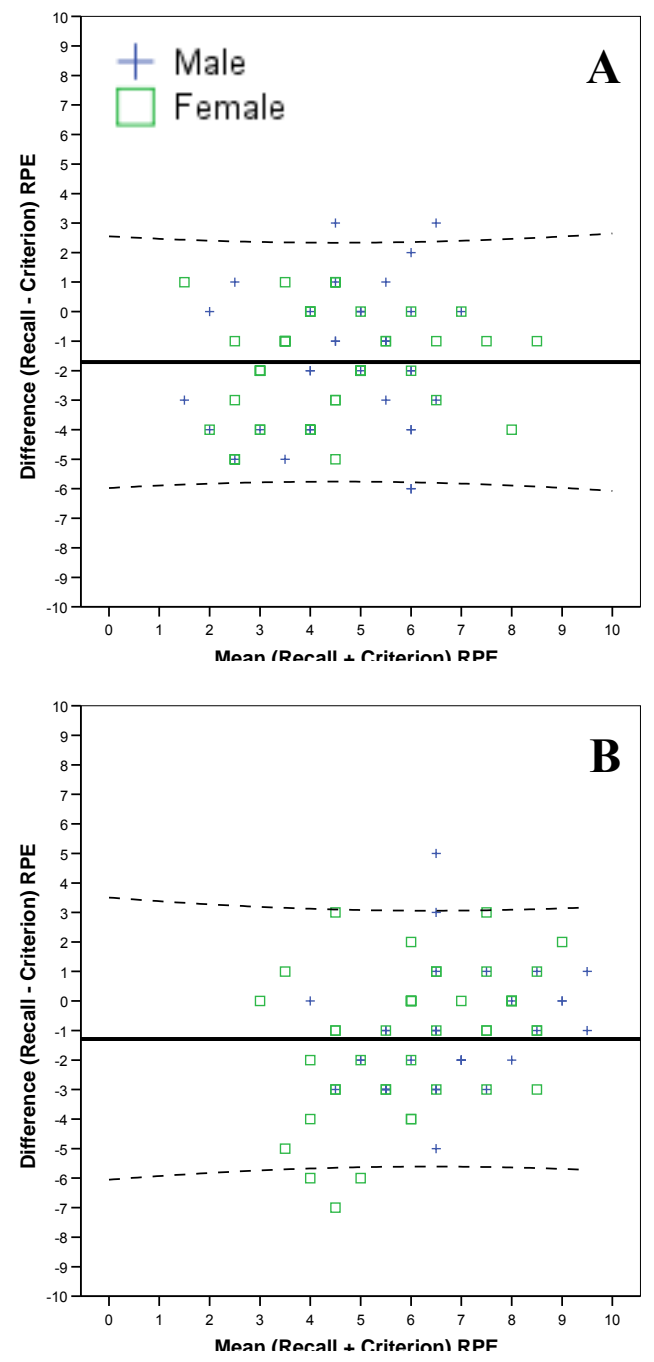

Figure 8. Bland-Altman Plots of (A) RPE-O and (B) RPE-A for

Two Intensities of Resistance Exercise 
Table 14. Frequency Distribution of the RPE Differences between Recall and Criterion Procedures for Resistance Exercise*

\begin{tabular}{ccc}
\hline Difference & RPE-O & RPE-A \\
\hline-7 & 0 & 1 \\
-6 & 2 & 2 \\
-5 & 5 & 2 \\
-4 & 12 & 3 \\
-3 & 7 & 17 \\
-2 & 15 & 11 \\
-1 & 16 & 15 \\
0 & 13 & 14 \\
1 & 7 & 9 \\
2 & 1 & 2 \\
3 & 2 & 3 \\
4 & 0 & 0 \\
5 & 0 & 1 \\
\hline
\end{tabular}

* Frequencies based on Bland-Altman Plots in Figure 8.

\subsection{ANALYSIS OF COVARIANCE}

\subsubsection{Cycle Exercise}

In an attempt to further explain significant differences found in the analysis of variance for cycle exercise, a three-factor [Procedure (Recall vs. Criterion) x Intensity (lower vs. higher) x Sex] analysis of covariance was conducted on RPE using frequency and duration of habitual exercise as the covariates. Separate analyses were conducted for the undifferentiated (RPE-O) and the differentiated ratings (RPE-L and RPE-C).

\subsubsection{Overall Body Responses}

The results of the analysis of covariance for RPE-O are presented in Table 15. After adjusting for both the frequency and duration of habitual exercise, there was not a significant main effect of procedure and sex on RPE-O. As expected, there was a significant main effect of intensity on 
RPE-O. Interaction effects of procedure $\mathrm{X}$ intensity, procedure $\mathrm{X}$ sex, intensity $\mathrm{X}$ sex, and procedure $\mathrm{X}$ intensity $\mathrm{X}$ sex were not significant on RPE-O.

Table 15. Results of the Analysis of Covariance for RPE-O during Cycle Exercise

\begin{tabular}{lcccc}
\hline Main Effects & df & $\mathbf{F}$ & $\boldsymbol{p}$ & $\boldsymbol{\eta}^{2}$ \\
\hline Procedure (P) & 1 & 0.161 & 0.691 & 0.004 \\
Intensity (I) & 1 & 9.935 & $0.003^{*}$ & 0.216 \\
Sex (S) & 1 & 1.385 & 0.247 & 0.037 \\
& & & & \\
Interaction Effects & & & & \\
\hline P x I & 1 & 0.813 & 0.373 & 0.022 \\
P x S & 1 & 0.697 & 0.409 & 0.019 \\
Ix S & 1 & 0.223 & 0.639 & 0.006 \\
P X I x S & 1 & 0.030 & 0.863 & 0.001 \\
& & & & \\
Error & 36 & & & \\
\hline
\end{tabular}

* Statistically significant (2-tailed)

\subsubsection{Leg Responses}

The results of the analysis of covariance for RPE-L are presented in Table 16. After adjusting for both the frequency and duration of habitual exercise, there was not a significant main effect of procedure and sex on RPE-L. As expected, there was a significant main effect of intensity on RPE-L. Interaction effects of procedure $\mathrm{X}$ intensity, intensity $\mathrm{X}$ sex, and procedure $\mathrm{X}$ intensity $\mathrm{X}$ sex were not significant for RPE-L. There was a significant interaction effect of procedure X sex (Appendix G.1). Post-hoc analysis indicated Recall RPE-L for males was not significantly different from females for the lower cycling intensity, $p=0.090$. However, Recall RPE-L was significantly higher for males than females for the higher cycling intensity, $p=0.015$. Post-hoc analysis did not indicate a difference in RPE-L between procedures. 
Table 16. Results of the Analysis of Covariance for RPE-L during Cycle Exercise

\begin{tabular}{lcccc}
\hline Main Effects & df & $\mathbf{F}$ & $\boldsymbol{p}$ & $\boldsymbol{\eta}^{\mathbf{2}}$ \\
\hline Procedure (P) & 1 & 0.037 & 0.848 & 0.001 \\
Intensity (I) & 1 & 7.918 & $0.008^{*}$ & 0.180 \\
Sex (S) & 1 & 2.448 & 0.126 & 0.064 \\
& & & & \\
Interaction Effects & & & & \\
\hline P x I & 1 & 1.297 & 0.262 & 0.035 \\
P x S & 1 & 4.959 & $0.032^{*}$ & 0.121 \\
Ix S & 1 & 0.198 & 0.659 & 0.005 \\
P x I x S & 1 & 0.002 & 0.967 & $<0.001$ \\
& & & & \\
Error & 36 & & & \\
\hline
\end{tabular}

* Statistically significant (2-tailed)

\subsubsection{Chest Responses}

The results of the analysis of covariance for RPE-C are presented in Table 17. After adjusting for both the frequency and duration of habitual exercise, there was not a significant main effect of procedure and sex on RPE-C. As expected, there was a significant main effect of intensity on RPE-C. Interaction effects of procedure $\mathrm{X}$ intensity, procedure $\mathrm{X}$ sex, intensity $\mathrm{X}$ sex, and procedure X intensity X sex were not significant for RPE-C.

Table 17. Results of the Analysis of Covariance for RPE-C during Cycle Exercise

\begin{tabular}{lcccr}
\hline Main Effects & df & $\mathbf{F}$ & $\boldsymbol{p}$ & \multicolumn{1}{c}{$\boldsymbol{\eta}^{\mathbf{2}}$} \\
\hline Procedure (P) & 1 & 0.918 & 0.344 & 0.025 \\
Intensity (I) & 1 & 7.100 & $0.011^{*}$ & 0.165 \\
Sex (S) & 1 & 0.329 & 0.570 & 0.009 \\
& & & & \\
Interaction Effects & & & & \\
\hline P x I & 1 & 2.021 & 0.164 & 0.053 \\
P x S & 1 & 0.003 & 0.957 & $<0.001$ \\
Ix S & 1 & 0.066 & 0.799 & 0.002 \\
P x I x S & 1 & $<0.001$ & 0.997 & $<0.001$ \\
& & & & \\
Error & 36 & & & \\
\hline * Statistically significant (2-tailed) & & &
\end{tabular}

* Statistically significant (2-tailed) 


\subsubsection{Resistance Exercise}

In an attempt to further explain significant differences found in the analysis of variance results for resistance exercise, a three-factor [Procedure (Recall vs. Criterion) x Intensity (light vs. heavy) $\mathrm{x}$ Sex] analysis of covariance was conducted on ratings of perceived exertion. The participants' frequency and duration of habitual exercise were used as the covariates in the analysis. Separate analyses were conducted for the undifferentiated (RPE-O) and the differentiated (RPE-A) ratings.

\subsubsection{Arm Responses}

The results of the analysis of covariance for RPE-A are presented in Table 18. After adjusting for the frequency and duration of habitual exercise, there was no significant main effect of procedure and intensity on RPE-A. There was a significant main effect of sex on RPE-A. This indicated that males reported a higher RPE-A than females. Interaction effects of procedure X intensity, procedure $\mathrm{X}$ sex, intensity $\mathrm{X}$ sex, and procedure $\mathrm{X}$ intensity $\mathrm{X}$ sex were not significant for RPEA.

Table 18. Results of the Analysis of Covariance for RPE-A during Resistance Exercise

\begin{tabular}{lcccr}
\hline Main Effects & df & $\mathbf{F}$ & $\boldsymbol{p}$ & \multicolumn{1}{c}{$\boldsymbol{\eta}^{\mathbf{2}}$} \\
\hline Procedure (P) & 1 & 0.002 & 0.966 & $<0.001$ \\
Intensity (I) & 1 & 1.390 & 0.246 & 0.037 \\
Sex (S) & 1 & 5.783 & $0.021^{*}$ & 0.138 \\
& & & & \\
Interaction Effects & & & & \\
\hline P x I & 1 & 2.672 & 0.111 & 0.069 \\
P x S & 1 & 0.009 & 0.925 & $<0.001$ \\
I X S & 1 & 2.333 & 0.135 & 0.061 \\
P x I x S & 1 & 2.405 & 0.130 & 0.063 \\
& & & & \\
Error & 36 & & & \\
* Statistically significant (2-tailed) & & &
\end{tabular}




\subsubsection{Overall Body Responses}

The results of the analysis of covariance for RPE-O are presented in Table 19. After adjusting for the frequency and duration of habitual exercise, there was no significant main effect of procedure, sex and intensity on RPE-O. Interaction effects of procedure X intensity, procedure $\mathrm{X}$ sex, intensity $\mathrm{X}$ sex, and procedure $\mathrm{X}$ intensity $\mathrm{X}$ sex were not significant for RPE-O.

Table 19. Results of the Analysis of Covariance for RPE-O during

Resistance Exercise

\begin{tabular}{lcccc}
\hline Main Effects & df & $\mathbf{F}$ & $\boldsymbol{p}$ & $\boldsymbol{\eta}^{\mathbf{2}}$ \\
\hline Procedure (P) & 1 & 0.051 & 0.822 & 0.001 \\
Intensity (I) & 1 & 2.013 & 0.165 & 0.053 \\
Sex (S) & 1 & 2.285 & 0.139 & 0.060 \\
& & & & \\
Interaction Effects & & & & \\
\hline P x I & 1 & 2.234 & 0.144 & 0.058 \\
P x S & 1 & 1.720 & 0.198 & 0.040 \\
I x S & 1 & 0.618 & 0.437 & 0.017 \\
P x I x S & 1 & 2.259 & 0.142 & 0.059 \\
& & & & \\
Error & 36 & & & \\
\hline * Statistically significant (2-tailed) & & &
\end{tabular}

\subsection{SUMMARY OF RESULTS}

Analyses of variance indicated that Recall and Criterion RPE-O, and -L, did not differ significantly at both cycle exercise intensities. Recall RPE-C was significantly different than Criterion RPE-C at both cycle exercise intensities. These findings held for both male and female subject groups. There was a significant interaction effect of procedure X sex for RPE-L. Posthoc analysis of this interaction indicated that Recall RPE-L for males did not significantly differ from females for the lower cycling intensity. However, Recall RPE-L was significantly higher for males than females for the higher cycling intensity. Post-hoc analysis indicated that RPE-L 
did not differ between procedures. There was also a significant interaction effect of procedure $\mathrm{X}$ intensity on RPE-C. Post-hoc analysis of this interaction indicated that Recall RPE-C did not significantly differ from the Criterion RPE-C for the lower cycling intensity but Recall RPE-C was significantly lower than the Criterion RPE-C for the higher cycling intensity.

After adjusting for the frequency and duration of habitual exercise, analyses of covariance indicated that RPE-O, -L, and $-\mathrm{C}$ did not differ between Recall and Criterion procedures for both cycle exercise intensities. Analysis of covariance also indicated that the significant interaction effect of procedure X sex on RPE-L remained. Post-hoc analysis of this interaction indicated that Recall RPE-L for males did not significantly differ from females for the lower cycling intensity bout but Recall RPE-L was significantly higher for males than females for the higher cycling intensity.

Analyses of variance indicated that the RPE-O and -A for the Recall procedure were significantly less than the Criterion procedure. After adjusting for the frequency and duration of habitual exercise, analyses of covariance indicated that RPE-O and -A did not differ between Recall and Criterion procedures for both resistance exercise intensities. 


\subsection{DISCUSSION, CONCLUSION, AND RECOMMENDATIONS}

\subsection{RECALLED RPE: CYCLE EXERCISE}

Physical activity questionnaires are important tools to quantify physical activity behaviors and to relate these behaviors to disease risk. However, of all the movement components that comprise physical activity, intensity is the most difficult to directly and objectively measure using questionnaire methodology. As such, the majority of physical activity questionnaires assess intensity using estimated metabolic units (i.e. METS). These estimated METS typically classify intensity of activity using normative values for healthy young to middle aged adults. At best, this method only provides approximations of the actual physical activity intensity.

An alternative means of assessing physical activity intensity using questionnaires employs the recalled RPE. Ratings of perceived exertion are known to be strongly correlated with $\mathrm{VO}_{2}$ and $\mathrm{HR}$ during aerobic exercise. Thus, RPE can be employed as a perceptual marker of physical activity intensity level. The rationale underlying this application is that the perceptual intensity varies directly with the relative metabolic rate. That is, for a given submaximal $\mathrm{VO}_{2}$, $\mathrm{RPE}$ is distributed as the inverse of $\mathrm{VO}_{2 \max }$ and as such will present as a positive function of the relative aerobic metabolic rate expressed as a percent of $\mathrm{VO}_{2 \max }$. Therefore, a rating of perceived exertion can be used as a measure of the relative physical activity intensity. As an example, Lee et al. ${ }^{1}$, reporting data from the Harvard Alumni Health Study, observed an inverse association between the relative intensity of physical activity as measured by recalled RPE and the level of 
disease risk. These data indicated that the beneficial health outcomes of physical activity were positively related to the relative exercise intensity as measured by recalled RPE. The basis of this relation holds that the relative exercise intensity as measured by recalled RPE reflects interindividual differences in aerobic fitness level.

Subsequent investigations have reported that the relative intensity of recreational activity as measured by recalled RPE can have a favorable impact on both gestational diabetes and preeclampsia risk $^{60,61}$. Both studies employed a recall paradigm to measure the relative intensity of the subject's prepregnancy recreational physical activity using the Borg CR-10 Perceived Exertion Scale ${ }^{60,61}$. It was reported that the relative intensity of recreational physical activity in the year before pregnancy was strongly and inversely related to subsequent preeclampsia risk ${ }^{60}$. In a similar study, using the same intensity recall procedure the perceived exertion during usual recreational physical activity in the year preceding pregnancy was strongly and inversely related to the risk of gestational diabetes mellitus. However, there is still limited published data on the validity of exertional recall to establish relative exercise intensities using questionnaire methodology.

In the present investigation, it was hypothesized that the perception of physical exertion (i.e. RPE-O, RPE-L, and RPE-C) experienced during the cycling portion of the Circuit Exercise Session and recalled 7-days later would not differ from the RPE estimated during a RecallCriterion Session that employed the same cycling intensities and duration. The mean RPE responses (Appendix F) were similar to those reported previously for aerobic exercise involving subjects with characteristics similar to those employed presently ${ }^{4,62}$.

Concurrent validation for the cycling component of the exertional recall questionnaire was first examined by correlating RPE with $\mathrm{VO}_{2}$ measured separately for each cycle intensity. A 
low-to-moderate correlation between $\mathrm{VO}_{2}$ and Recall RPE-L $(\mathrm{r}=0.275$ and 0.409$)$. In addition, there was a weak relation between $\mathrm{VO}_{2}$ and Recall RPE-O $(\mathrm{r}=0.090$ and 0.241$)$ and no relation between $\mathrm{VO}_{2}$ and Recall RPE-C $(\mathrm{r}=-0.005$ and 0.010$)$. Similarly, the correlations between Criterion RPEs and $\mathrm{VO}_{2}(\mathrm{r}=0.097$ to 0.433$)$ were low-to-moderate for the separate cycle intensities. These low correlations were likely due to the limited variability observed for both $\mathrm{RPE}$ and $\mathrm{VO}_{2}$ when measured at a single exercise intensity. When data from the two cycling intensities were combined in the correlation analyses, the relation between Recall RPEs and $\mathrm{VO}_{2}$ $(r=0.258$ to 0.569$)$ were generally similar to the relation between Criterion RPEs and $\mathrm{VO}_{2}(r=$ 0.432 to 0.633$)$. The correlations using combined data indicated that both the Recall and the Criterion RPE variables increased concurrently with increases in $\mathrm{VO}_{2}$ as aerobic exercise intensity increased. At best these correlations provide only moderate validity as they are somewhat lower than the expected $\mathrm{r}=0.70$ that would normally be found in studies using similar variables. These results are consistent with those of Schafer et al. ${ }^{62}$ whom validated a seven day exertional recall procedure for recreationally active young adults performing treadmill walking and running. This previous investigation observed moderate correlations between $\mathrm{VO}_{2}$ and Recall RPE-O, - $\mathrm{L}$, and -C ( $\mathrm{r}=0.54$ to 0.56$)$.

Data analysis using factorial comparisons indicated that the exertional recall procedure evidenced construct validity for the two different cycle intensities within the exercise circuit. The Recall RPE-O did not differ from the Criterion RPE-O indicating that seven days following exercise both females and males were able to recall their overall level of exertion experienced during the cycling bouts. Similarly, the RPE-L did not differ between the Recall and the Criterion procedures for either cycle intensity. However, Recall RPE-C was lower than the Criterion RPE-C. The mean difference between the Recall and the Criterion RPE-C ratings was 
less than one RPE unit. As such, the observed statistical difference may have comparatively little effect on the application of the exertional recall questionnaire in public health settings. Lastly, both the undifferentiated and differentiated ratings of perceived exertion were higher for the higher cycle intensity. As such, Recall RPE distinguished between two distinctly different exercise intensities and associated physiological responses.

For RPE-L, there was a significant interaction effect of procedure $\mathrm{X}$ sex. During the higher cycling intensity in which the male subjects had a mean RPE-L of 8.1 females had a mean RPE-L of 6.9. There was no significant difference during the lower cycling intensity. The exact underlying mechanisms for the occurrence of this interaction are unknown especially considering that exercise was performed at the same $\% \mathrm{VO}_{2 \max }$ for both sex groups. The current investigation used relative intensities $\left(\sim \% \mathrm{VO}_{2 \max }\right)$ determined separately for each subject for both the lower and the higher cycling intensities. Nevertheless, one possible explanation is that the higher cycling intensity was performed at a comparatively higher percentage of peak power output for males than females. The higher percent of muscle mass contraction may have signaled a more intense RPE-L. In contrast, Schafer et al. ${ }^{62}$ did not observe sex differences in any RPE variable where exercise intensities were constant and not adjusted to achieve a specified percent of maximal oxygen uptake.

It is possible that the frequency and duration of habitual exercise may be confounding variables in the Recall procedure. Individuals who report comparatively greater frequency and duration of habitual exercise may have greater perceptual precision and less "memory fade" when recalling exertion using a structured questionnaire. An ANCOVA using frequency and duration of habitual exercise as the covariates did not change the data interpretations for cycle exercise but the findings were strengthened as the p-values increased. The only effect that was 
still significant after adjusting for regular exercise frequency and duration was the procedure $\mathrm{X}$ sex interaction for RPE-L. The results from the ANCOVA support the suggestion that greater frequency and duration of habitual exercise may improve the precision of recalled exertional perceptions.

\subsection{RECALLED RPE: RESISTANCE EXERCISE}

It was also hypothesized that the perception of physical exertion (i.e. RPE-O and RPE-A) experienced during the resistance component of the Circuit Exercise Session and recalled 7-days later would not differ from the RPE estimated by the subjects during the Recall-Criterion Session that employed the same resistance intensities. The mean RPE responses (Appendix F) were similar to previous investigations for resistance exercise involving subjects with characteristics similar to those employed presently ${ }^{3,67}$.

The present findings were in contrast to the hypothesis. Construct validity was not evident for the recall of exertional perceptions associated with a previously performed bicep curl exercise. The Recall RPE-O and -A were significantly lower than Criterion RPE-O and -A at both resistance intensities. As such, the Recall RPEs were consistently under-estimated for both the lighter and the heavier resistances. Although the total volume of weight lifted was similar between the 65\% 1-RM (i.e. 12 repetitions) and the 90\% 1-RM (i.e. 6 repetitions) there was a significant difference in both the differentiated and undifferentiated ratings between the two resistance intensities. The Recall RPE indicated that the exertion was greater when lifting the heavier weight than the lighter weight seven days previously. Recall RPE was able to distinguish between lower and higher resistance exercise intensities even when total weight was equal. This 
is a positive feature of the exertional recall procedure. When frequency and duration of habitual exercise were controlled in the ANCOVA, there was not a significant difference of procedure on RPE-O or $-\mathrm{A}$ for the resistance exercise bouts. This is important as ANCOVA indicated that the recall procedure can be used if mechanisms are in place to account for frequency and duration of habitual exercise. The results from the ANCOVA support the suggestion that a greater frequency and duration of habitual exercise may improve the precision of recalled exertional perceptions for upper body resistance exercise.

It is possible that the length of the resistance exercise sets and/or volume of active muscle mass may have mitigated the perceived exertion response observed presently. Subjects performed just one set of bicep curl that required approximately 40 seconds. Under conditions of comparatively short muscle contractions, the Recall procedure may not be valid. However, when the subjects performed non-weight bearing aerobic activity for 5 minutes the Recall procedure was valid. A longer period of exposure to the exercise stimulus may sharpen "perceptual memory" of exertion associated with resistance exercise. Another possible mitigating factor in explaining the under-estimation of Recall RPE for resistance exercise is the activation of muscle mass ${ }^{63}$, 64 . Bicep curl exercise requires unilateral limb involvement and activation of a comparatively small muscle mass. Cycling exercise, where the Recall procedure was valid, requires greater muscle mass activation than the bicep curl. As such, exertion associated with increased time on stimulus and/or with greater muscle mass activation may evidence greater precision when recalled. 


\subsection{RECALLED RPE: SESSION}

A secondary hypothesis of the investigation held that perception of physical exertion (i.e. RPESession) experienced during the entire Circuit Exercise Session and recalled 7-days later would not differ from the RPE-Session estimated by the subjects immediately following a RecallCriterion Session that employed the same circuit protocol.

The current investigation found that Recall RPE-Session (6.7) was significantly higher than Criterion RPE-Session (6.3). The mean difference between the Recall and the Criterion RPE-Session was less than one RPE unit. As such, the observed statistical difference may have comparatively little effect on application of the exertional recall questionnaire when a global rating of exertion for an entire session is involved. That is, individuals would be expected to evidence only a slight over-estimation in their Recall RPE for an entire circuit protocol consisting of multiple modes of stationary cycling and resistance exercise.

Previous research has examined the validity of the Session-RPE for intermittent treadmill walking and running, as well as intermittent and continuous stationary cycling and resistance

exercise $^{59,65-68}$. The results from the present investigation extend previous reports by examining RPE-Session following a multi-modal circuit exercise protocol. The importance of RPE-Session as a post-exercise global measure of the relative intensity experienced during an entire aerobic and resistance circuit exercise program has potential public health benefits. Multi-modal exercises often characterize physical activity patterns in the general population and in sports related exercise training. Health-fitness conditioning, leisure time activities, and competitive athletic training can involve multiple modes that activate both aerobic and anaerobic energy pathways. The validation of a recalled RPE-Session for multi-modal exercises may lend to greater public health applications in evaluating the appropriateness of exercise prescription and 
determining health benefits of habitual exercise. A recalled RPE-Session may be a useful alternative for assessing the relative exercise intensity in questionnaire methodology as only one response item is needed for an entire exercise session.

\subsection{UNDERESTIMATION AND OVERESTIMATION}

Both under-estimation and over-estimation of Recall RPE responses were identified by BlandAltman plots with a 95\% CI. These plots identified over- and under-estimation using a sex grouping effect. The Bland-Altman plots generally demonstrated a good inter-procedure agreement between Recall and Criterion RPEs for both females and males during cycle exercise. However, the plot reveals a slight under-estimation of RPE-O, -L, and -C by the Recall procedure. The upper and lower limits of agreement as defined by the $95 \%$ CI can only be used as a general marker. Using a "generally accepted rule" it is expected that a difference of less than one RPE unit on a 10 category scale may not have practical significance in exercise prescription and intensity self-regulation. Hence, reconfiguration of the Bland-Altman plots for cycle exercise (Figure 9) indicated roughly half of the subjects were consistently within one RPE unit (shaded region) between the Recall and the Criterion procedures for (A) RPE-O, (B) RPE-L, and (C) RPE-C. 

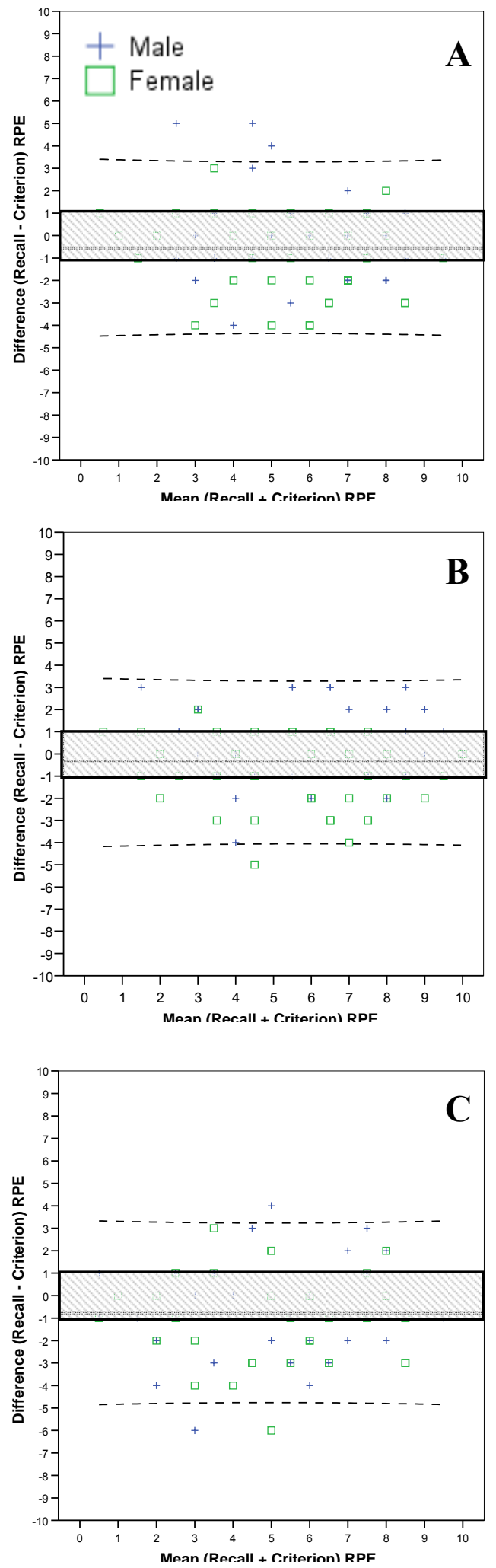

Figure 9. Reconfigured Bland-Altman Plots for Cycle Exercise 
When the group data for the cycling bouts were examined, the mean difference indicated a slight under-estimation of RPE-O, $-\mathrm{L}$, and $-\mathrm{C}$ by the Recall procedure. However, when sex specific data were considered, males tended to over-estimate their Recall RPE-O and -L whereas females tended to under-estimate their Recall RPE-O and -L. For example: 4 of the 5 subjects who over-estimated their Recall RPE-O were males, and 8 of the 9 subjects who over-estimated their Recall RPE-L were males. Additionally, 13 of the 18 subjects who under-estimated their Recall RPE-O were females, and 10 of the 14 subjects who under-estimated their Recall RPE-L were females. There was also a general trend for those who over-estimated their Recall RPE-O to also over-estimate their Recall RPE-L. There was no sex trend for Recall RPE-C as comparatively equal numbers of females and males over- or under-estimated their Recall RPE.

Bland-Altman plots demonstrated poor agreement between Recall and Criterion RPEs for females and males during resistance exercise. Reconfiguration of the Bland-Altman plots for resistance exercise (Figure 10) indicated only a comparatively small number of subjects consistently were within one RPE unit (shaded region) difference between the Recall and the Criterion procedures for (A) RPE-O, and (B) RPE-A. 

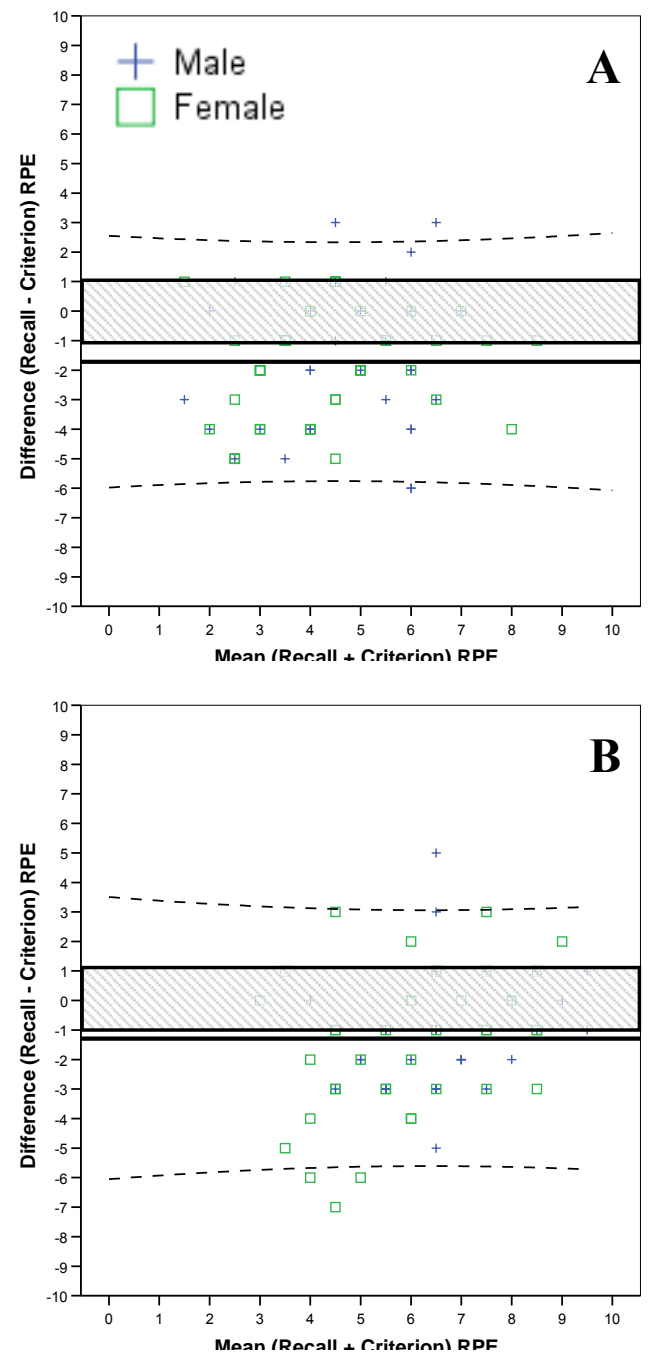

Figure 10. Reconfigured Bland-Altman Plots for Resistance Exercise

Both the mean of the sample and the majority of the individual data points lie outside the limits of "generally accepted" (i.e. \pm 1 RPE) agreement indicating a marked under-estimation of the Recall RPE during resistance exercise. Frequency trends were similar for resistance exercise as that found for cycle exercise. 


\subsection{SUMMARY}

In conclusion, recreationally active females and males with no prior knowledge of the OMNI RPE Scale were able to recall their perceived exertion experienced during non-weight bearing (cycle) aerobic exercise performed seven days previously. The findings held for both the undifferentiated overall body rating and differentiated ratings for legs and chest. The Recall procedure is valid for non-weight bearing aerobic exercise. However, the subjects underestimated their Recall RPE-O and $-\mathrm{A}$ for upper body resistance exercise. These responses question validity of the Recall RPE procedure for single joint arm resistance exercise. Additionally, the Recall RPE-Session was found to be valid for a combined aerobic and resistance exercise circuit protocol.

As evidenced by the ANCOVA, frequency and duration of habitual exercise may be confounding variables in the recall procedure. Those individuals that participated in greater frequency and duration of exercise may have greater exertional precision in recalling the subjective intensity of exertion. Implications of these findings may require that application of the exertional recall procedure may have to a priori categorize individuals according to their frequency and duration of habitual exercise.

Use of a perceptual intensity measure in a physical activity recall questionnaire could provide valuable information in evaluating health outcomes of regular aerobic exercise. Such information can be used to support mounting evidence that links both the quantity (volume) and quality (intensity) of physical activity participation with life long health. 


\subsection{RECOMMENDATIONS}

In light of the current findings, future investigations should focus on the following research questions. For aerobic exercise modalities, follow-on investigations should examine exertional recall validity for 1) extended durations, 2) additional exercise modes, 3) diversified population subsets, 4) varied recall time periods, and 5) sex of participants.

1. The current findings strongly support the validity of an exertional recall procedure for non-weight bearing aerobic exercise lasting 5 minutes. Future research should examine the validity of the same exertional recall procedure for treadmill walking and running and stationary cycling of durations longer than 5 minutes. Durations of 20 to 30 minutes or longer are recommended as they are typically prescribed for aerobic exercise programs.

2. To date, the exertional recall procedure has been used in treadmill walking and running and stationary cycling. Additional modes of aerobic exercise should also be examined i.e. elliptical exercise, stepping, rowing, and swimming.

3. The subjects for both the Schafer et al. ${ }^{62}$ study and the current investigation were recreationally active between the ages of 18 and 35 years. Future research should examine the validity of the exertional recall questionnaire procedure for both sedentary and elite/competitive population subsets as well as a younger and older individuals performing walking, running, and cycling exercise programs. 
4. The most commonly employed physical activity questionnaires ask the individual to recall physical activity performed 1 to 7 days previously with some procedures measuring recall up to a year after performance. Future research should consider the validity of the exertional recall procedure for post-exercise periods that range from one to 52 weeks.

5. The current investigation found that Recall RPE-L was greater in males than in females for the higher cycling intensity. Research should further examine the underlying mechanisms that may contribute to this sex differences. It is possible that sex differences in recalled exertional intensity are associated with a comparatively greater relative intensity of muscular contraction or greater activated muscle mass for males.

Perceptual memory of exertional intensity experienced during previously performed resistance exercise may be influenced by 1) time on exercise stimulus, and 2) single- versus multi-joint limb involvement.

1. It is possible that the significant differences between Recall and Criterion RPE for resistance exercise may be attributed to the comparatively short duration of the exercise stimulus, where each set lasted approximately 40 seconds. A 40 second resistance exercise period may be insufficient for the subject to accurately recall their perceived exertion. Thus, future research should investigate the validity of an exertional recall procedure 
for longer duration resistance exercises such as that found in a circuit training program that consists of multiple sets at a given intensity.

2. The current investigation examined the validity of exertional recall in resistance exercise using a dominant arm bicep curl. Greater total muscle mass activation and/or greater limb involvement may be necessary for the subject to accurately recall perceived exertion when assessed 7 days following performance of resistance exercise. Future research should investigate exertional recall for a variety of resistance exercises ranging from single joint to multi-joint action such as but not limited to leg extensions, squats, and lunges.

Additionally, future research should expand on the validity of the Recall RPE-Session for 1) extended durations, and 2) additional modes of exercise.

1. The RPE-Session was examined 5 minutes post-exercise. The validity of recalled RPE-Session should be examined for post-exercise measurement periods in excess of 5 minutes in duration.

2. Recalled RPE-Session was found to be valid for an exercise circuit protocol consisting of stationary cycling and bicep curl exercise. This occurred despite the observation that the Recall RPE for the resistance exercise component of the circuit protocol was not valid. Future research should examine the predominant exercise mode (i.e. aerobic or resistance) that contributes to recalled RPE-Session in mixed mode exercise circuits. 
APPENDIX A

SUBJECT RECRUITMENT PACKET 


\section{A.1 PHYSICAL ACTIVITY READINESS QUESTIONNAIRE}


ID \#

\section{University of Pittsburgh \\ Center for Exercise and Health-Fitness Research}

\section{Physical Activity Readiness Questionnaire (PAR-Q)}

Now I am going to ask you a few questions to determine if you are eligible to complete the stationary cycle exercise ...

1. Has your doctor ever said that you have a heart condition and that you should only do physical activity recommended by a doctor?

No _ _ Yes _ _ If yes, specify:

2. Do you feel pain in your chest when you do physical activity?

No _ Y Yes _ _ If yes, specify:

3. In the past month, have you had chest pain when you were not doing physical activity? No _ Y Yes _ If yes, specify:

4. Do you lose your balance because of dizziness or do you ever lose consciousness?

No __ Yes __ If yes, specify:

5. Do you have a bone or joint problem that could be made worse by a change in your physical activity?

No __ Yes__ If yes, specify:

6. Is your doctor currently prescribing drugs (for example, water pills) for a blood pressure or heart condition?

No _ Y Yes _ If yes, specify:

7. Do you know of any other reason why you should not do physical activity?

No __ Yes _ _ If yes, specify: 


\section{A.2 SUBJECT RECRUITMENT FLYER}




\section{Opportunity to Learn about Your Physical Fitness through Participation in a Research Study}

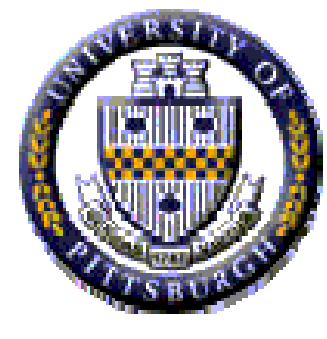

The University of Pittsburgh Department of Health and Physical Activity is now recruiting healthy, recreationally active males or females (18-35 years old) for a research study designed to look at how our body burns calories and your feelings of exercise.

\section{Each participant will complete:}

3 exercise sessions consisting of stationary cycling and biceps curl (45 minutes or less will be required for each session).

\section{To be eligible you must:}

1. Be healthy

2. Currently participate in 30 to 60 minutes of aerobic (i.e. running, sport clubs, intramurals) and/or resistance exercise 2 to 3 times a week.

3. Have not participated in collegiate or professional sports.

Upon completion, subjects will receive $\$ 25.00$
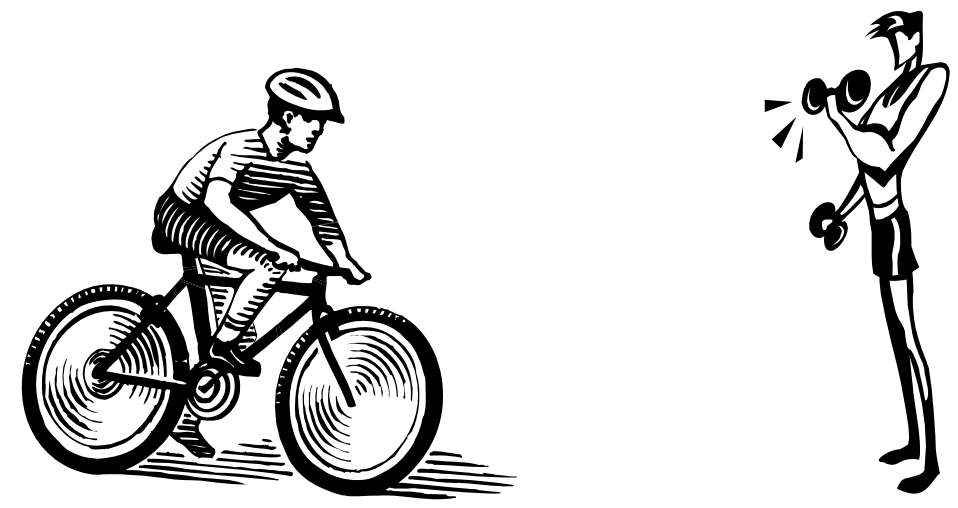

For more information and to see if you qualify, call: 412-648-8252 or email Michael Gallagher at migst27@pitt.edu 
APPENDIX B

OMNI-RPE SCALES 


\section{B.1 OMNI-RPE FOR CYCLE EXERCISE}

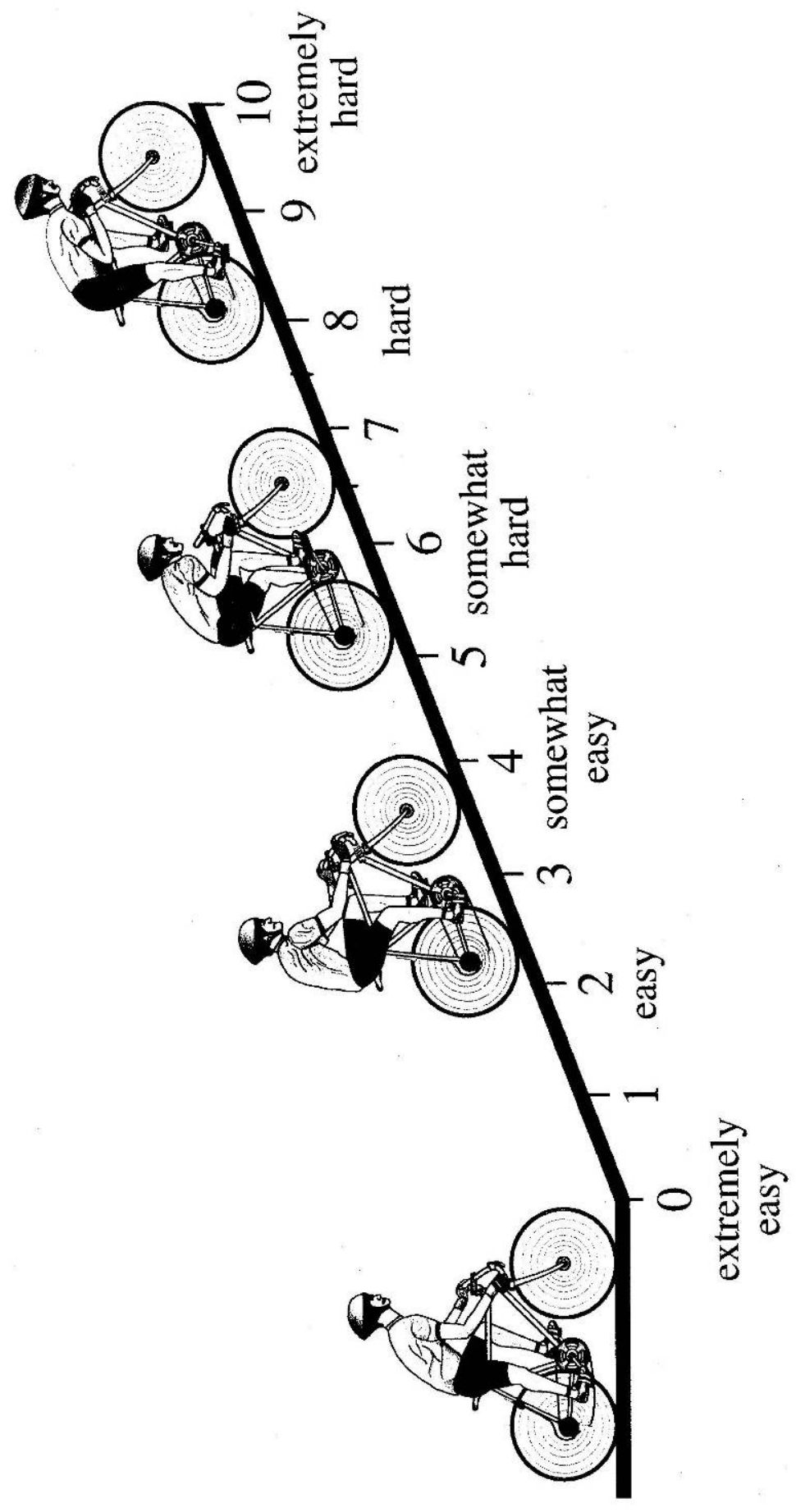


B.2 OMNI-RPE FOR RESISTANCE EXERCISE

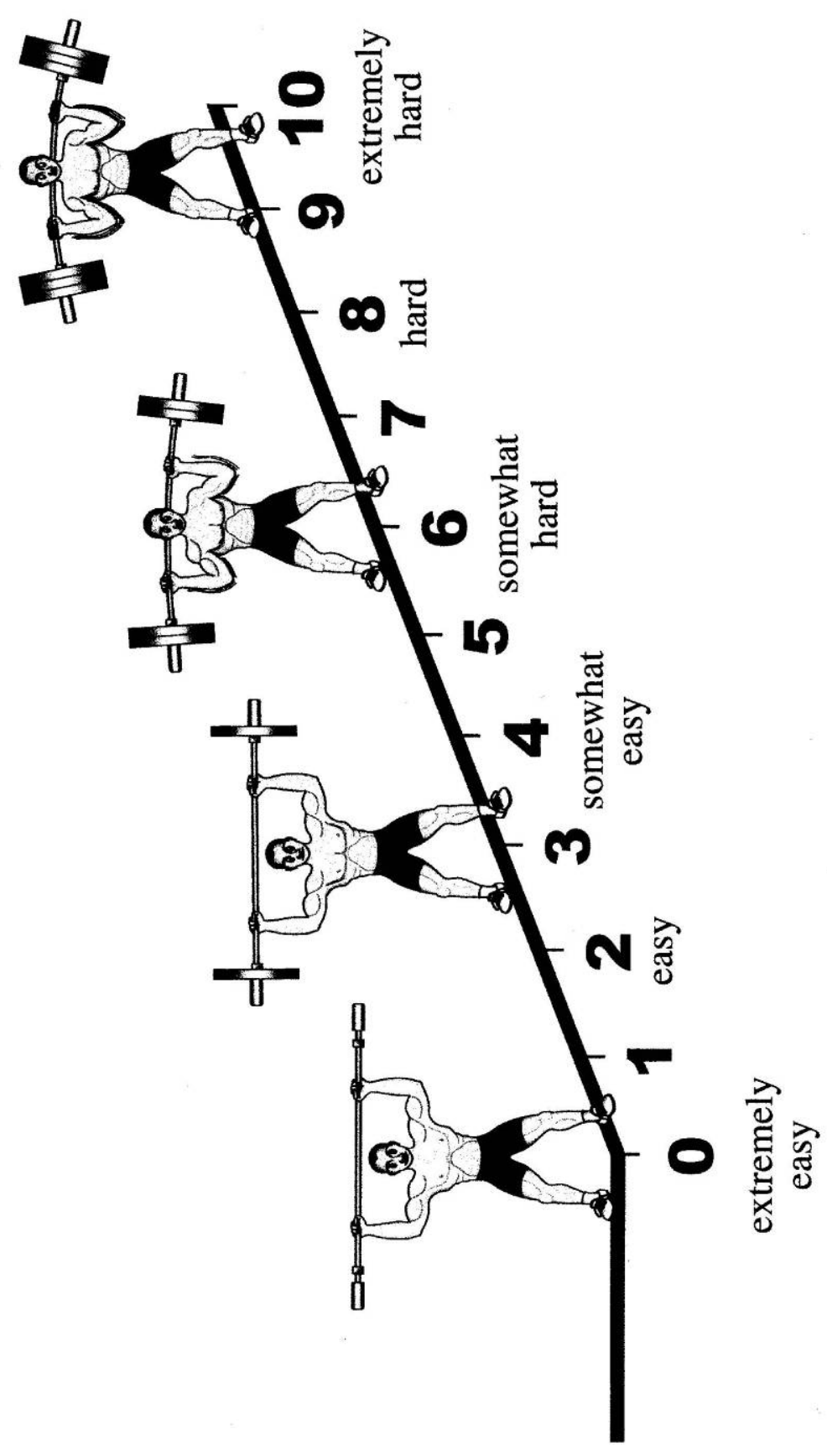

87 
APPENDIX C

RECALL QUESTIONNAIRES 


\section{C.1 SESSION RECALL QUESTIONNAIRE}
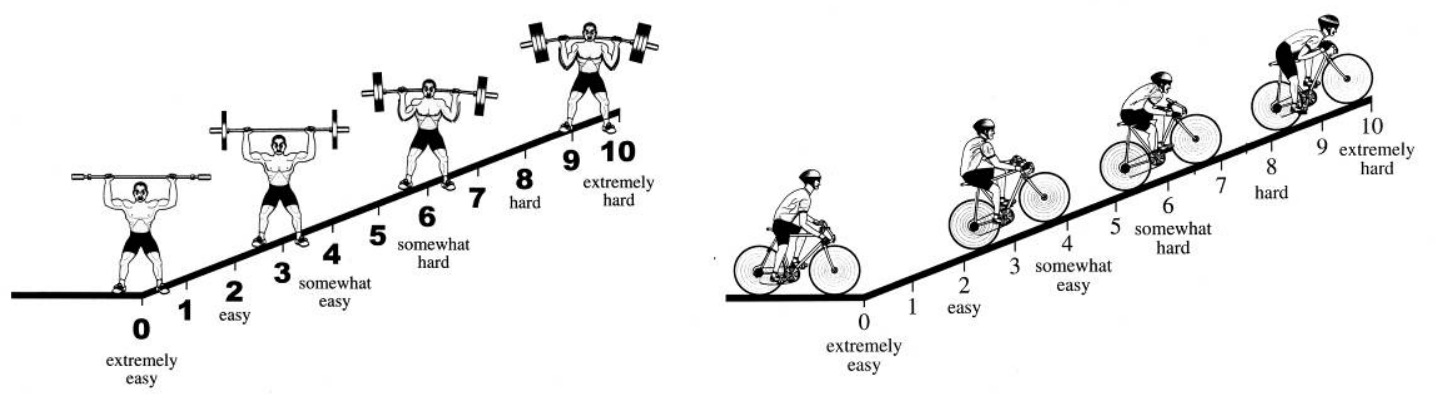

Think about the exercises that you performed in the circuit program one week (7 days) ago. Please use this picture scale to rate the level of effort, strain, discomfort and/or fatigue that you felt during the entire exercise circuit.

Using numbers from the above effort scale:

1. Rate your effort during the entire exercise circuit.

a. Your overall body

2. Which exercise influenced your rating of effort the most for the entire exercise circuit?
a. Cycle
b. Resistance 


\section{C.2 CYCLE EXERCISE RECALL QUESTIONNAIRE}

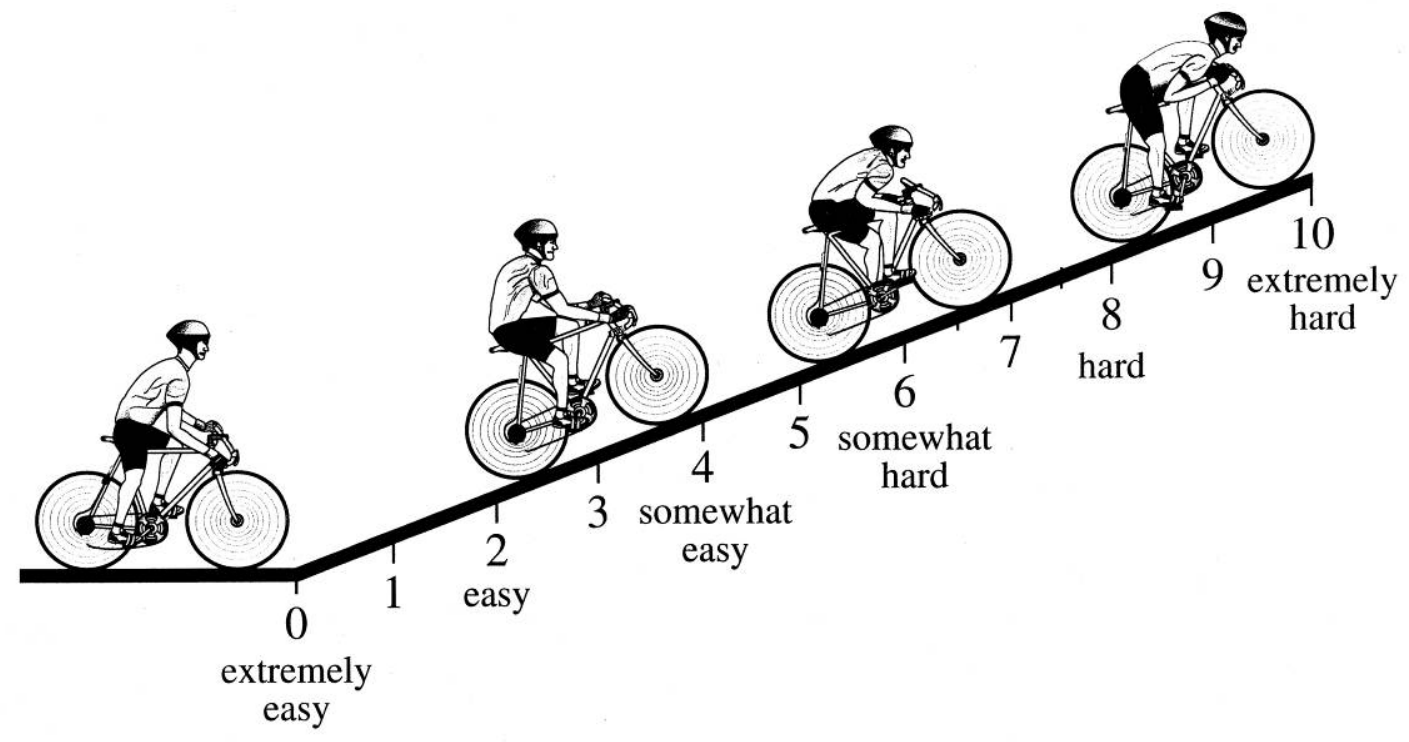

Think about the exercise that you performed on the stationary cycle in the circuit program one week (7 days) ago. Please use this picture scale to rate the level of effort, strain, discomfort and/or fatigue that you felt during the cycling exercise.

Using numbers from the above effort scale:

1. Rate your effort during the flat cycle ride.

b. Your overall body

b. Your legs

c. Your chest

2. Rate your effort during the uphill cycle ride.
c. Your overall body
b. Your legs
c. Your chest 


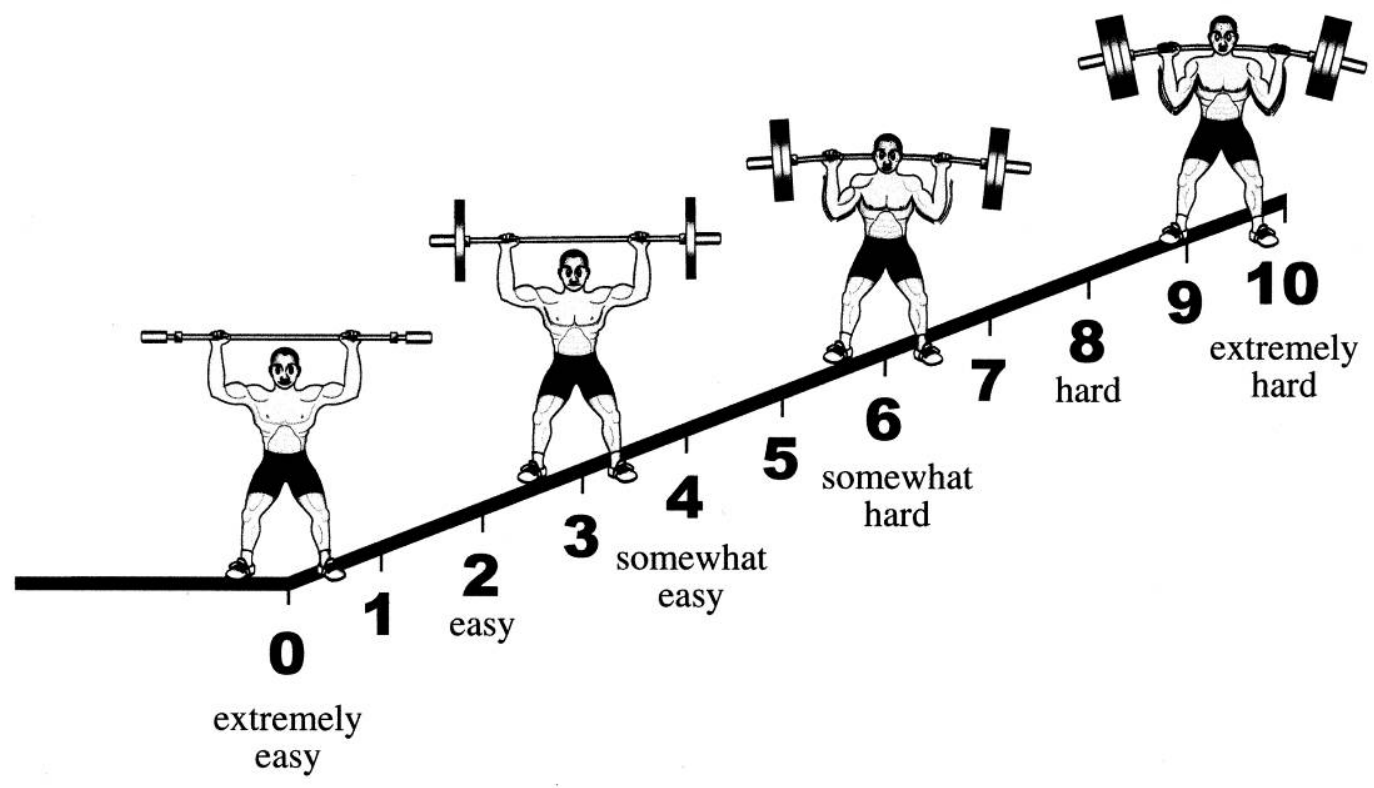

Think about the resistance/lifting exercise that you performed during the circuit program one week (7 days) ago. Please use this picture scale to rate the level of effort, strain, discomfort and/or fatigue that you felt during the bicep curl exercise.

Using numbers from the above effort scale:

1. Rate your effort during the "lighter weight" resistance station.
a. Your overall body
b. Your arm

2. Rate your effort during the "heavier weight" resistance station.
d. Your overall body
b. Your arm 
APPENDIX D

OMNI SCALE INSTRUCTIONS 


\section{D.1 OMNI - CYCLE INSTRUCTIONS}

We would like you to ride on a bicycle ergometer. Please use the numbers on this scale to tell us how your body feels when cycling. Look at the person at the bottom of the hill who is just starting to ride the bicycle. If you feel like this person when you are cycling, the exertion will be EXTREMELY EASY. In this case, your rating should be a number zero. Now look at the person who is barely able to ride a bicycle up the hill. If you feel like this person when cycling, the exertion will be EXTREMELY HARD. In this case, your rating should be a number 10. If you feel somewhere between Extremely Easy (0) and Extremely Hard (10) then give a number between 0 and 10 . We will ask you to give a number that tells how your whole body feels, how your chest and breathing feels, and how your legs feel. Remember, there are no right or wrong numbers. Use both the pictures and words to help you select a number. Use any of the numbers to tell how you feel when cycling. 


\section{D.2 OMNI - RESISTANCE INSTRUCTIONS (BICEPS CURL)}

We would like you to lift a weight using an exercise called the bicep curl. Please use the numbers on this scale to tell us how your body feels when lifting the weight. Look at the person on the left side of the scale who is just starting to perform a bicep curl (lift). If you feel like this person when you are lifting, the exertion will be EXTREMELY EASY. In this case, your rating should be a number zero. Now look at the person on the right side of the scale who is barely able to perform a bicep curl (lift). If you feel like this person when lifting, the exertion will be EXTREMELY HARD. In this case, your rating should be a number 10. If you feel somewhere between Extremely Easy (0) and Extremely Hard (10) then give a number between 0 and 10 . We will ask you to give a number that tells how your whole body feels and how your active muscle (bicep) feels. Remember, there are no right or wrong numbers. Use both the pictures and words to help you select a number. Use any of the numbers to tell how you feel when lifting the weight. 
APPENDIX E

MODIFIED ASTRAND PROTOCOL 
ID:

\section{Modified Astrand Cycle Ergometer Test}

Date:

Sex:

Age:

Height (in): Weight (lbs):

APMHR: $\quad$ Resting Heart Rate (bom):

Warm-Up: $2 \mathrm{~min}$ at $300 \mathrm{kgm} / \mathrm{min} \quad$ Pedaling Rate $=50 \mathrm{mpm}$

Males:

\begin{tabular}{|c|c|c|c|c|c|c|c|c|c|c|c|c|c|c|c|}
\hline Stage & \multicolumn{3}{|c|}{1} & \multicolumn{3}{|c|}{2} & \multicolumn{3}{|c|}{3} & \multicolumn{3}{|c|}{4} & \multicolumn{3}{|c|}{5} \\
\hline Time (min) & 1 & 2 & 3 & 1 & 2 & 3 & 1 & 2 & 3 & 1 & 2 & 3 & 1 & 2 & 3 \\
\hline $\begin{array}{c}\text { Resistance } \\
\text { (kg) } \\
\text { (rpm) }\end{array}$ & & $\begin{array}{l}2.0 \\
50\end{array}$ & & & $\begin{array}{l}3.0 \\
50\end{array}$ & & & $\begin{array}{l}4.0 \\
50\end{array}$ & & & $\begin{array}{l}5.0 \\
50\end{array}$ & & & $\begin{array}{l}6.0 \\
50\end{array}$ & \\
\hline kgmimin & & 600 & & & 900 & & & 1200 & & & 1500 & & & 1800 & \\
\hline Watts & & 100 & & & 150 & & & 200 & & & 250 & & & 300 & \\
\hline $\begin{array}{c}\text { Heart Rate } \\
\text { (bpm) }\end{array}$ & & & & & & & & & & & & & & & \\
\hline
\end{tabular}

Females:

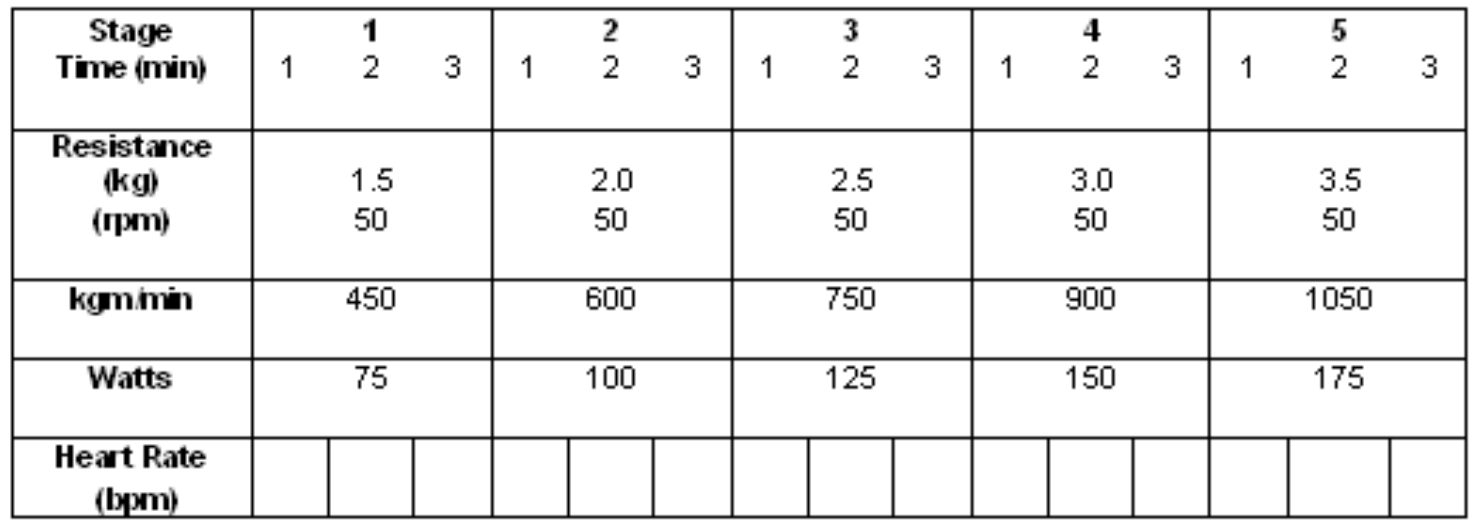


APPENDIX F

MEAN RPE DESCRIPTIVES 
Females: Cycle Exercise (Mean $\pm \mathrm{SD}$ )

\begin{tabular}{|c|c|c|c|c|c|c|}
\hline & \multicolumn{3}{|c|}{ Lower (Flat) Intensity } & \multicolumn{3}{|c|}{ Higher (Uphill) Intensity } \\
\hline Procedure & 0 & $\mathrm{~L}$ & $\mathrm{C}$ & 0 & $\mathbf{L}$ & $\mathrm{C}$ \\
\hline Recall & $3.40 \pm 1.87$ & $3.77 \pm 2.09$ & $3.72 \pm 2.03$ & $6.23 \pm 1.72$ & $6.86 \pm 1.64$ & $6.05 \pm 2.15$ \\
\hline Criterion & $4.14 \pm 2.61$ & $4.59 \pm 2.58$ & $4.18 \pm 2.44$ & $7.23 \pm 1.45$ & $7.95 \pm 1.46$ & $7.23 \pm 1.38$ \\
\hline
\end{tabular}

$\underline{\text { Females: Resistance Exercise (Mean } \pm \mathrm{SD} \text { ) }}$

\begin{tabular}{|c|c|c|c|c|}
\hline & \multicolumn{2}{|c|}{ Lighter Weight } & \multicolumn{2}{|c|}{ Heavier Weight } \\
\hline Procedure & 0 & $A$ & 0 & $A$ \\
\hline Recall & $2.68 \pm 1.73$ & $3.91 \pm 1.97$ & $4.50 \pm 1.65$ & $7.05 \pm 1.46$ \\
\hline Criterion & $4.73 \pm 1.58$ & $6.27 \pm 1.49$ & $5.27 \pm 1.67$ & $7.23 \pm 1.57$ \\
\hline
\end{tabular}

$\underline{\text { Males: Cycle Exercise (Mean } \pm \mathrm{SD} \text { ) }}$

\begin{tabular}{l|ccc|ccc|}
\multicolumn{2}{c}{} & \multicolumn{2}{c}{ Lower (Flat) Intensity } & \multicolumn{3}{c}{ Higher (Uphill) Intensity } \\
\cline { 2 - 4 } \multicolumn{1}{c}{} & & & & & & \\
\hline Procedure & 0 & L & C & 0 & L & C \\
\hline Recall & $4.28 \pm 1.87$ & $4.94 \pm 2.24$ & $3.33 \pm 2.33$ & $6.83 \pm 1.58$ & $8.06 \pm 1.76$ & $5.39 \pm 2.77$ \\
Criterion & $4.22 \pm 1.99$ & $4.44 \pm 1.95$ & $3.67 \pm 2.22$ & $7.17 \pm 2.01$ & $7.94 \pm 1.70$ & $6.44 \pm 2.25$ \\
\hline
\end{tabular}

Males: Resistance Exercise (Mean \pm SD)

\begin{tabular}{l|cc|cc|}
\cline { 3 - 5 } \multicolumn{2}{c}{ Lighter Weight } & \multicolumn{2}{c}{ Heavier Weight } \\
\cline { 2 - 5 } \multicolumn{1}{c}{ Procedure } & 0 & $\mathrm{~A}$ & 0 & $\mathrm{~A}$ \\
\hline Recall & $3.22 \pm 1.93$ & $5.06 \pm 1.55$ & $4.33 \pm 2.03$ & $7.06 \pm 1.76$ \\
Criterion & $5.44 \pm 1.65$ & $6.89 \pm 1.37$ & $6.28 \pm 1.64$ & $7.78 \pm 1.35$ \\
\hline
\end{tabular}


APPENDIX G

PLOTS OF THE INTERACTIONS 


\section{G.1 CYCLE EXERCISE}

\section{G.1.1 PROCEDURE X SEX INTERACTION for RPE-L (data derived from ANOVA)}

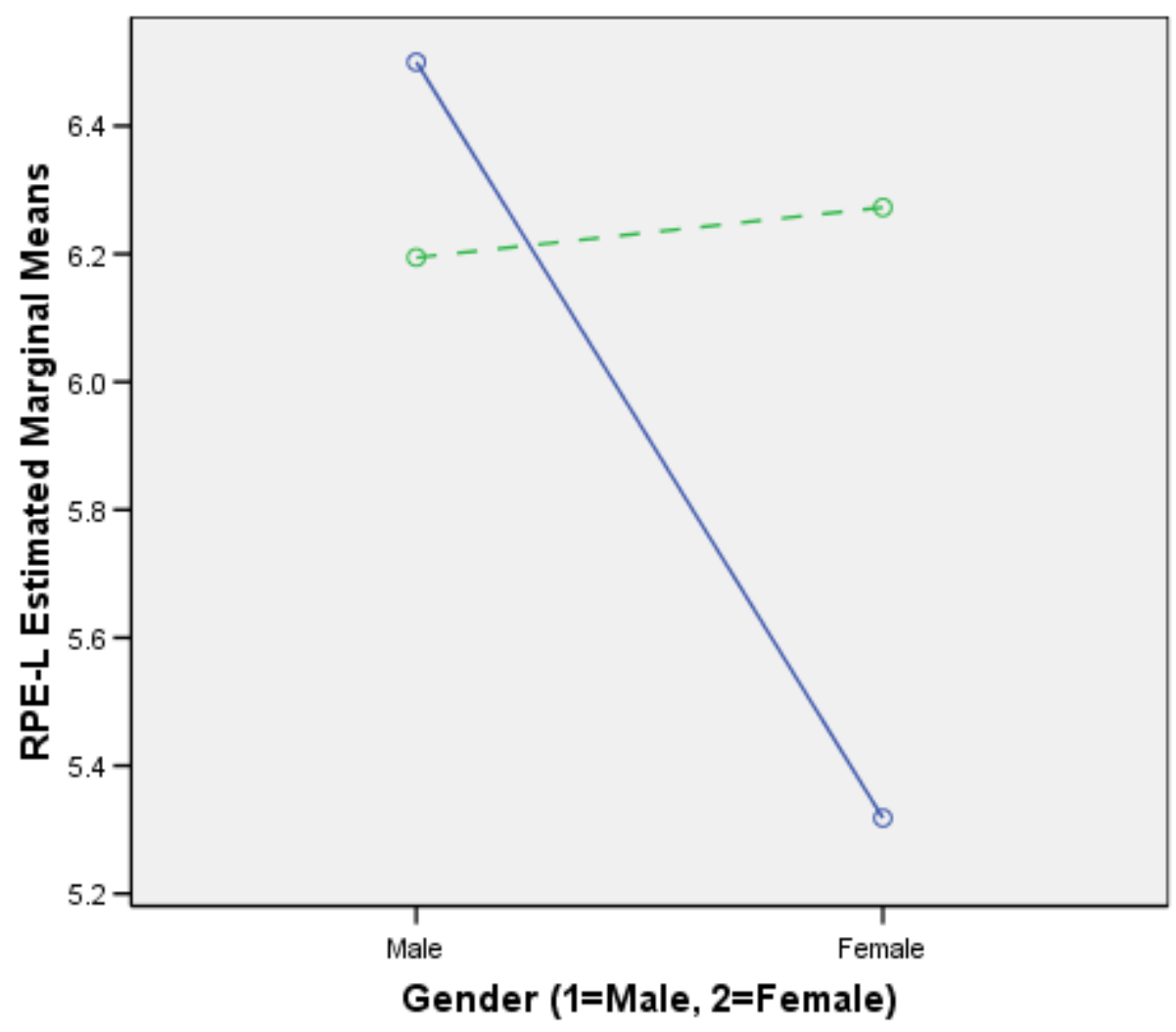

Procedure

$-1$

$--2$

Procedure: $1=$ Recall, $2=$ Criterion 


\section{G.1.2 PROCEDURE X INTENSITY INTERACTION for RPE-C (data derived from}

ANOVA)

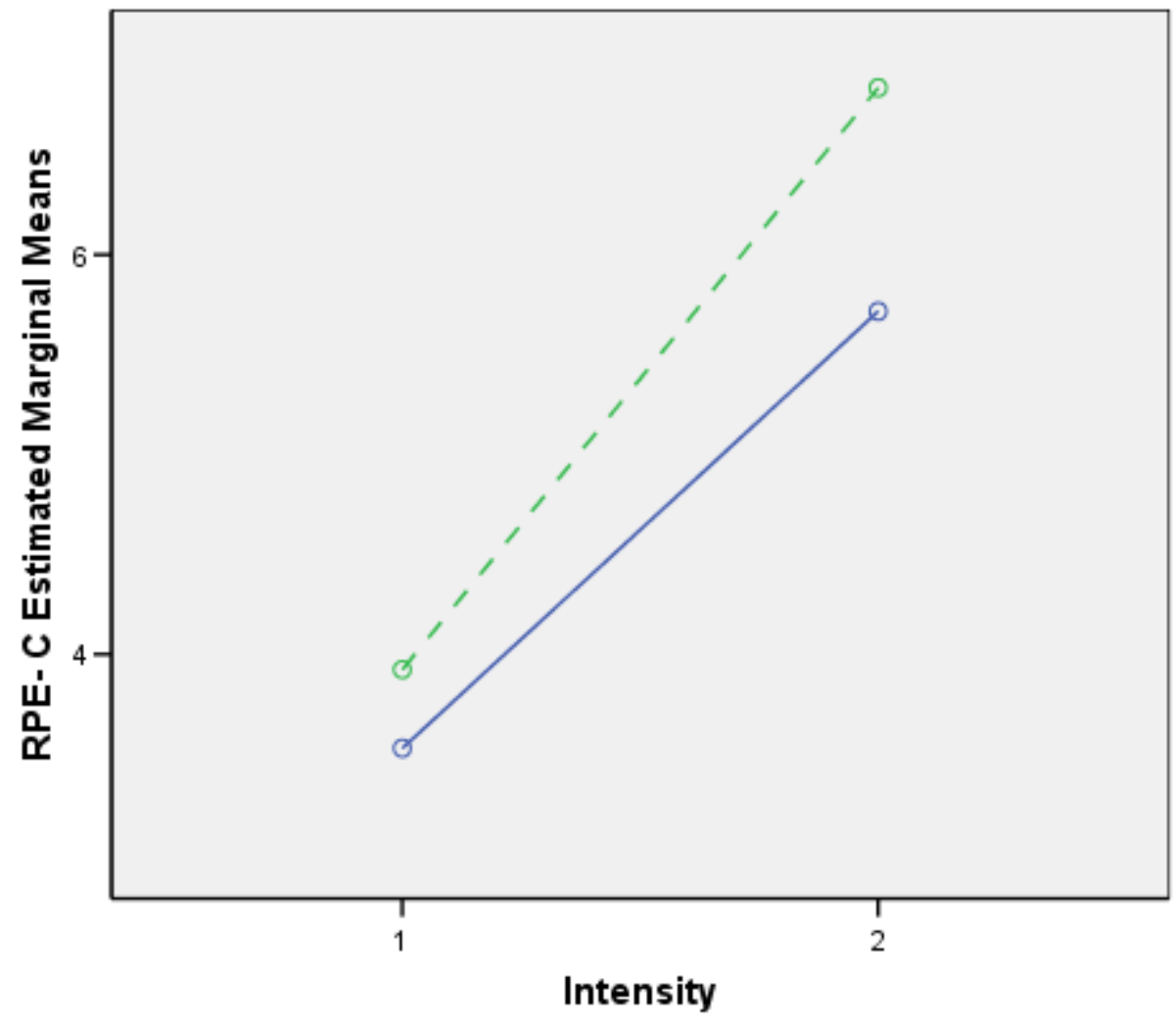

Procedure

$-1$

$--2$

Intensity: 1 = Lower (Flat) Intensity, 2 = Higher (Uphill) Intensity

Procedure: 1 = Recall, $2=$ Criterion 


\section{G.1.3 PROCEDURE X SEX INTERACTION for RPE-L (data derived from ANCOVA)}

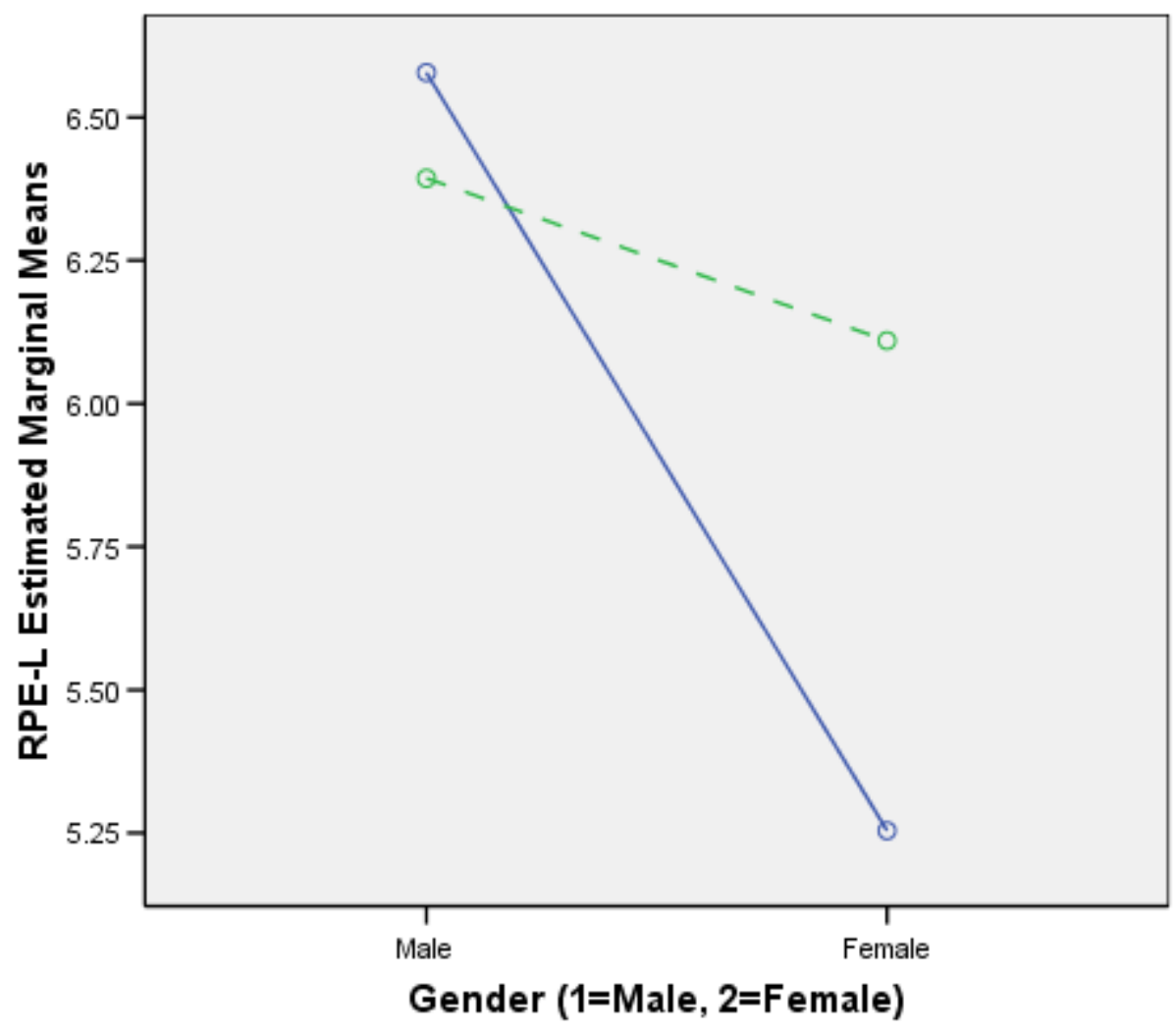

Procedure

$-1$

$--2$

Procedure: $1=$ Recall, $2=$ Criterion 


\section{G.2 RESISTANCE EXERCISE}

G.2.1 PROCEDURE X INTENSITY INTERACTION for RPE-A (data derived from ANOVA)

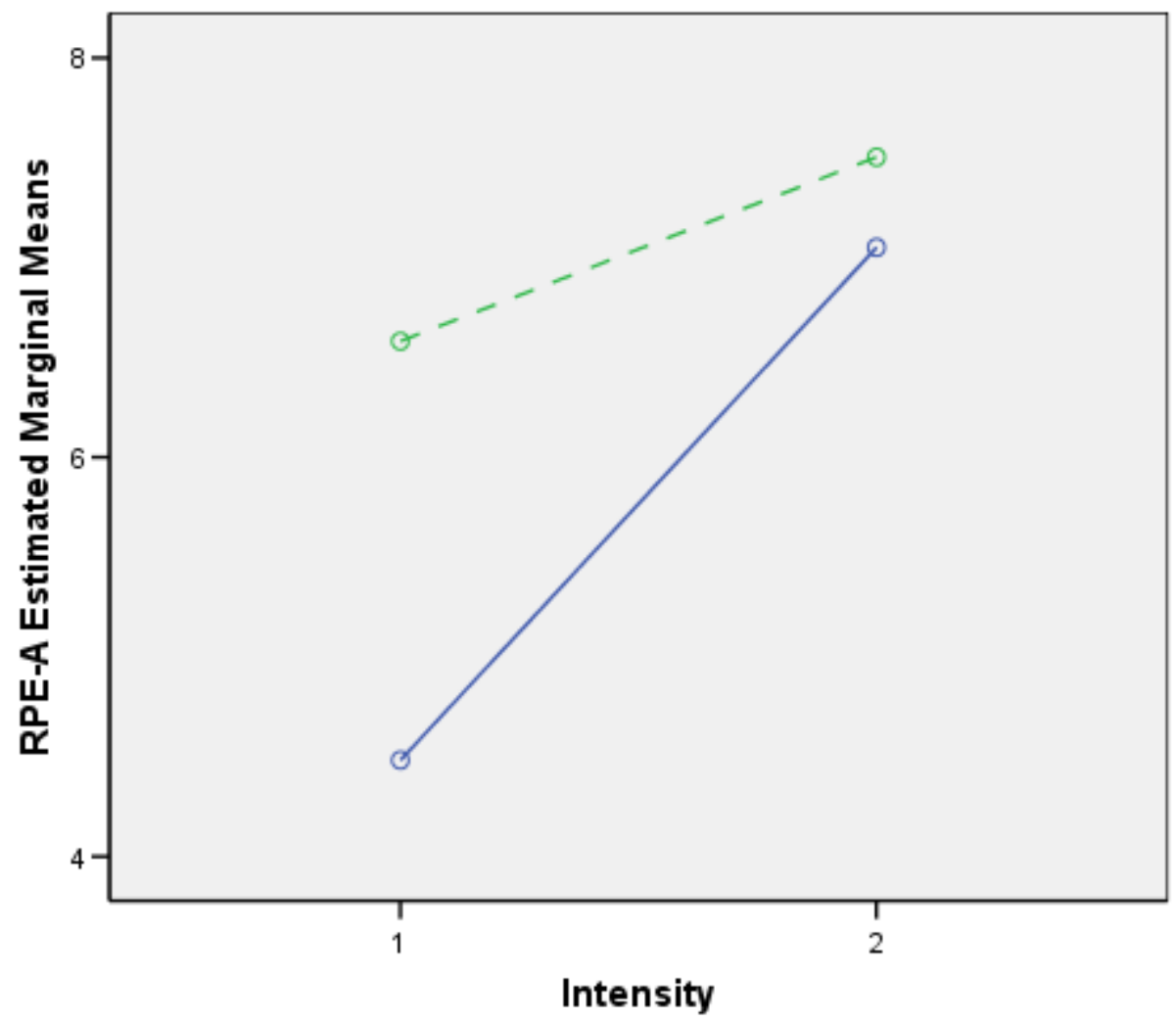

Procedure
-1
--2

Intensity: 1 = Lighter Weight, 2 = Heavier Weight

Procedure: $1=$ Recall, $2=$ Criterion 
G.2.2 PROCEDURE X INTENSITY INTERACTION for RPE-O (data derived from ANOVA)

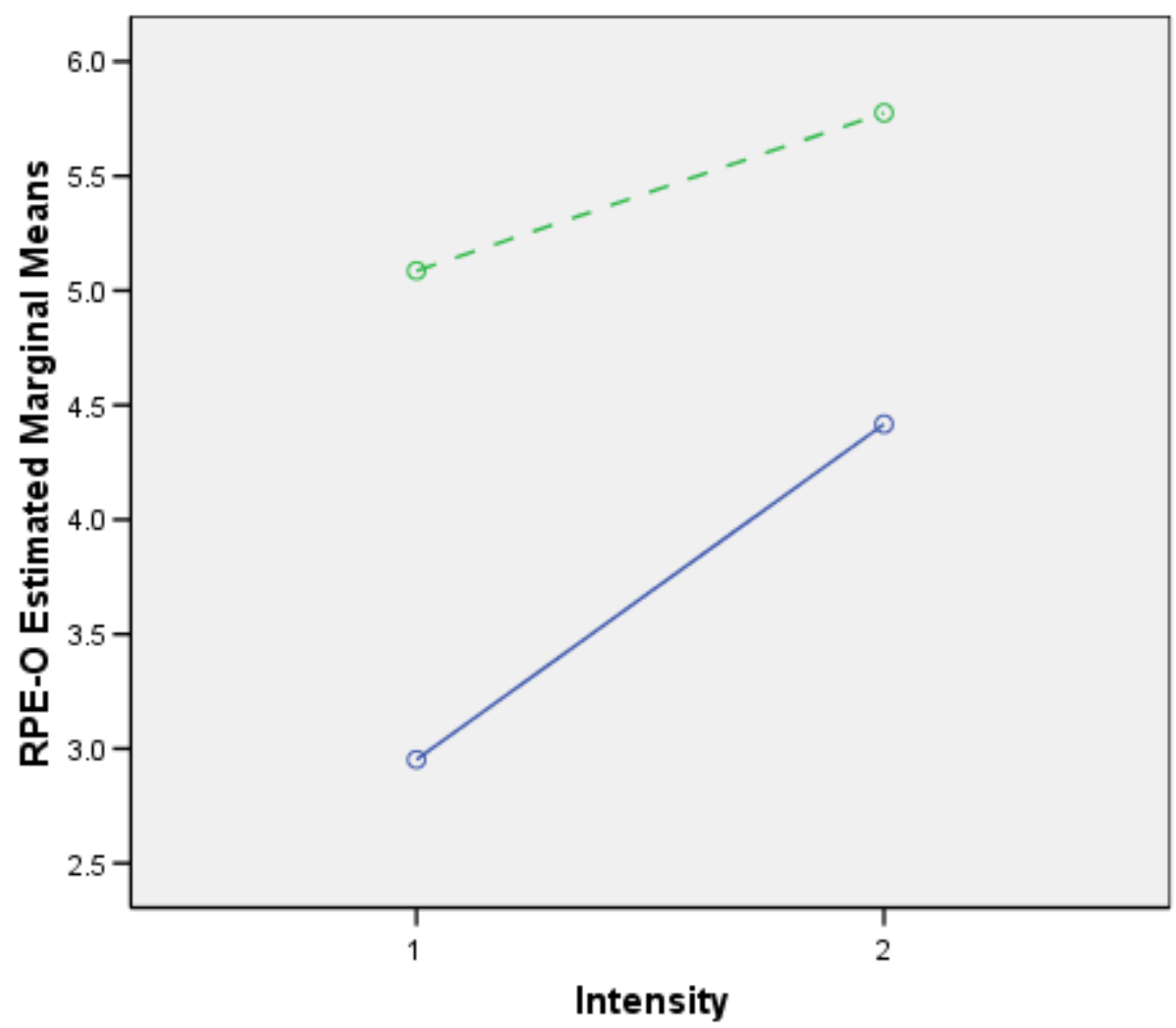

Procedure
-1
--2

Intensity: 1 = Lighter Weight, $2=$ Heavier Weight

Procedure: $1=$ Recall, $2=$ Criterion 


\section{APPENDIX H}

BLAND-ALTMAN PLOTS OF STANDARDIZED RPE SCORES 


\section{H.1 CYCLE EXERCISE}

\section{H.1.1 RPE-O}

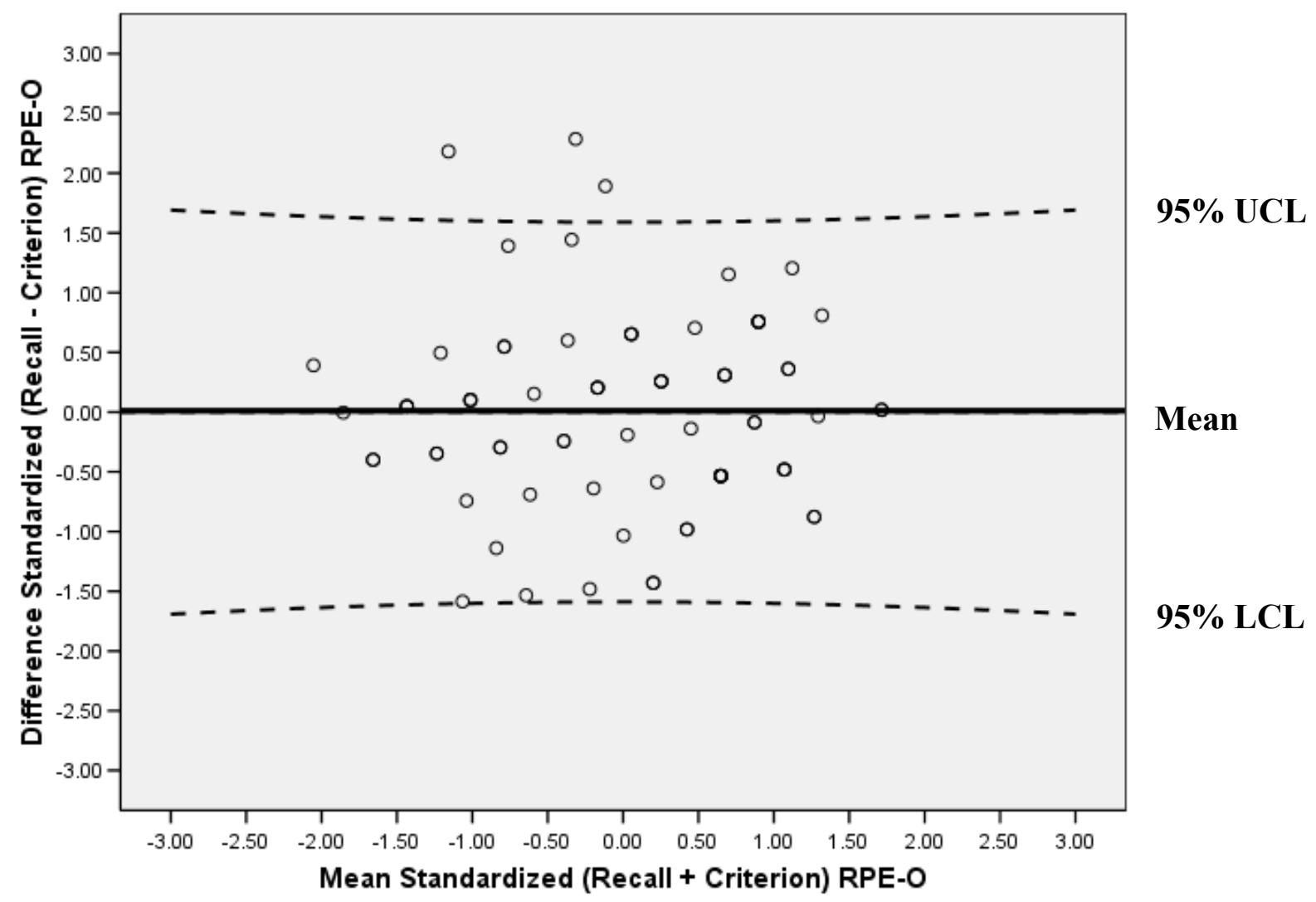

The mean difference (or bias) is indicated by a solid line. The 95\% confidence interval ( $\pm 2 \mathrm{SD}$; upper and lower limits of agreement) is indicated by dashed lines. Additionally, the range displayed on the $y$-axis represents the maximum possible RPE difference between the Recall and Criterion procedures.

UCL: 95\% Upper Confidence Limit

LCL: 95\% Lower Confidence Limit 

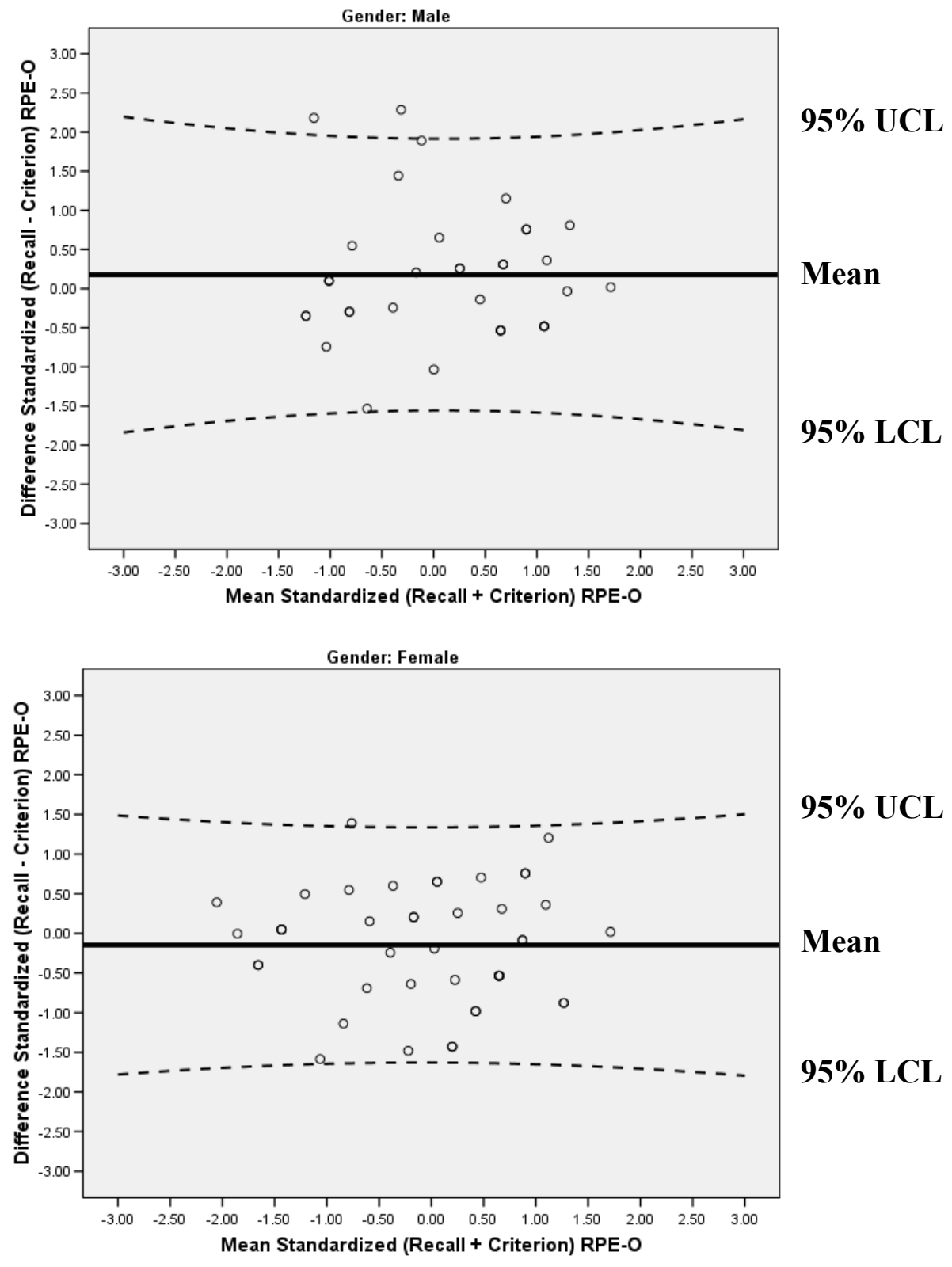

UCL: 95\% Upper Confidence Limit

LCL: 95\% Lower Confidence Limit 


\section{H.1.2 RPE-L}

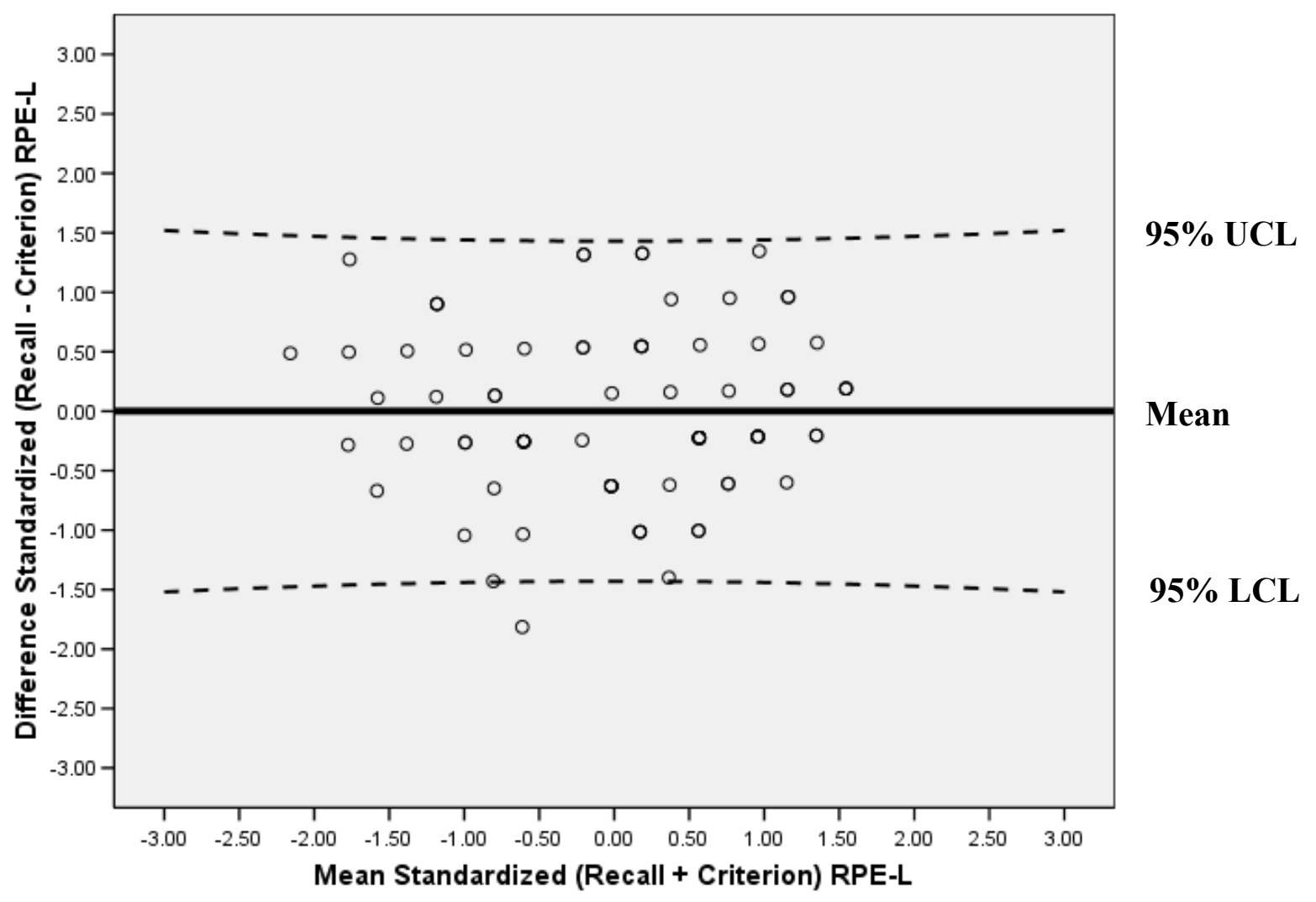

UCL: 95\% Upper Confidence Limit

LCL: 95\% Lower Confidence Limit 

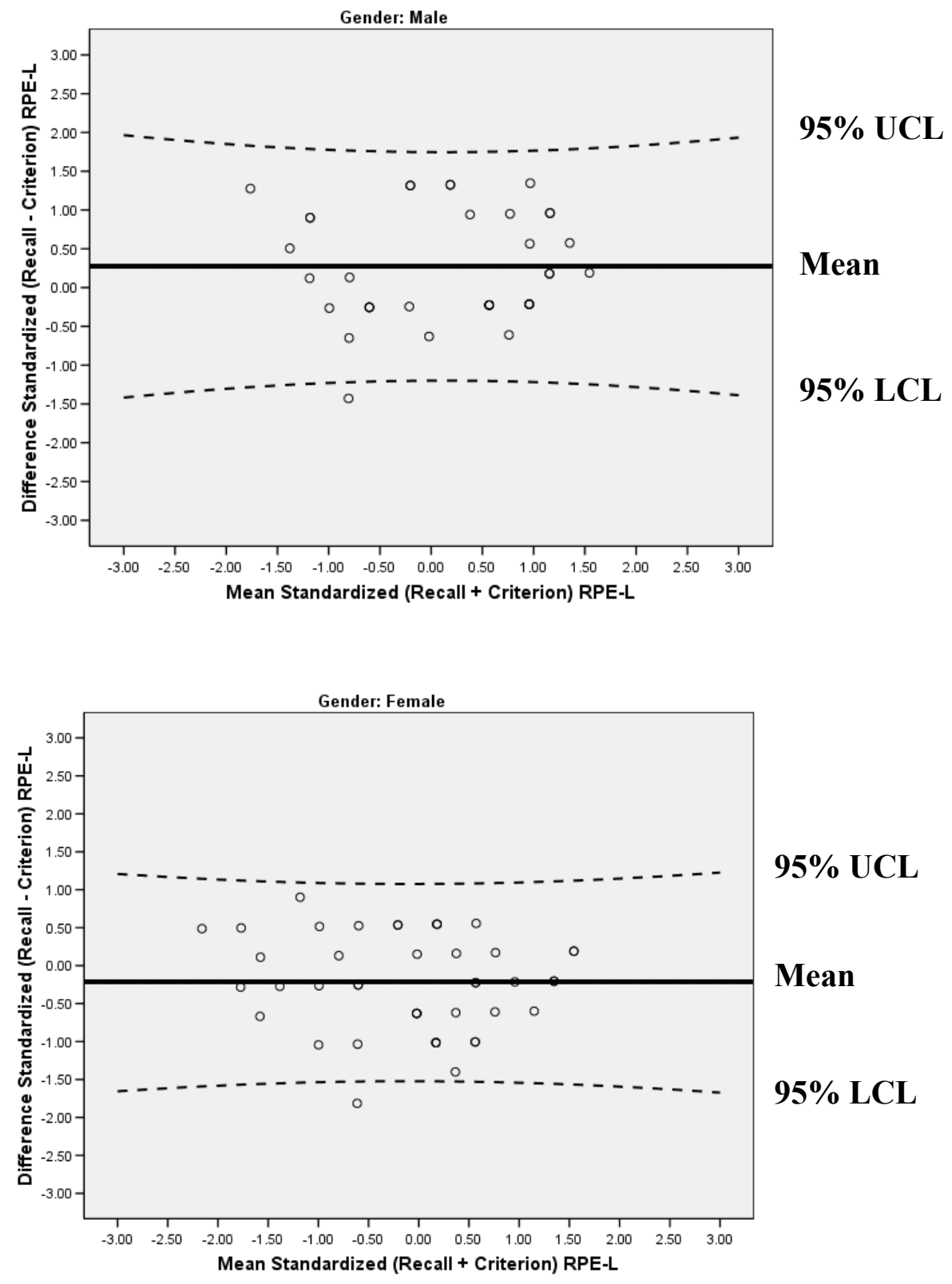

UCL: 95\% Upper Confidence Limit

LCL: 95\% Lower Confidence Limit 


\section{H.1.3 RPE-C}

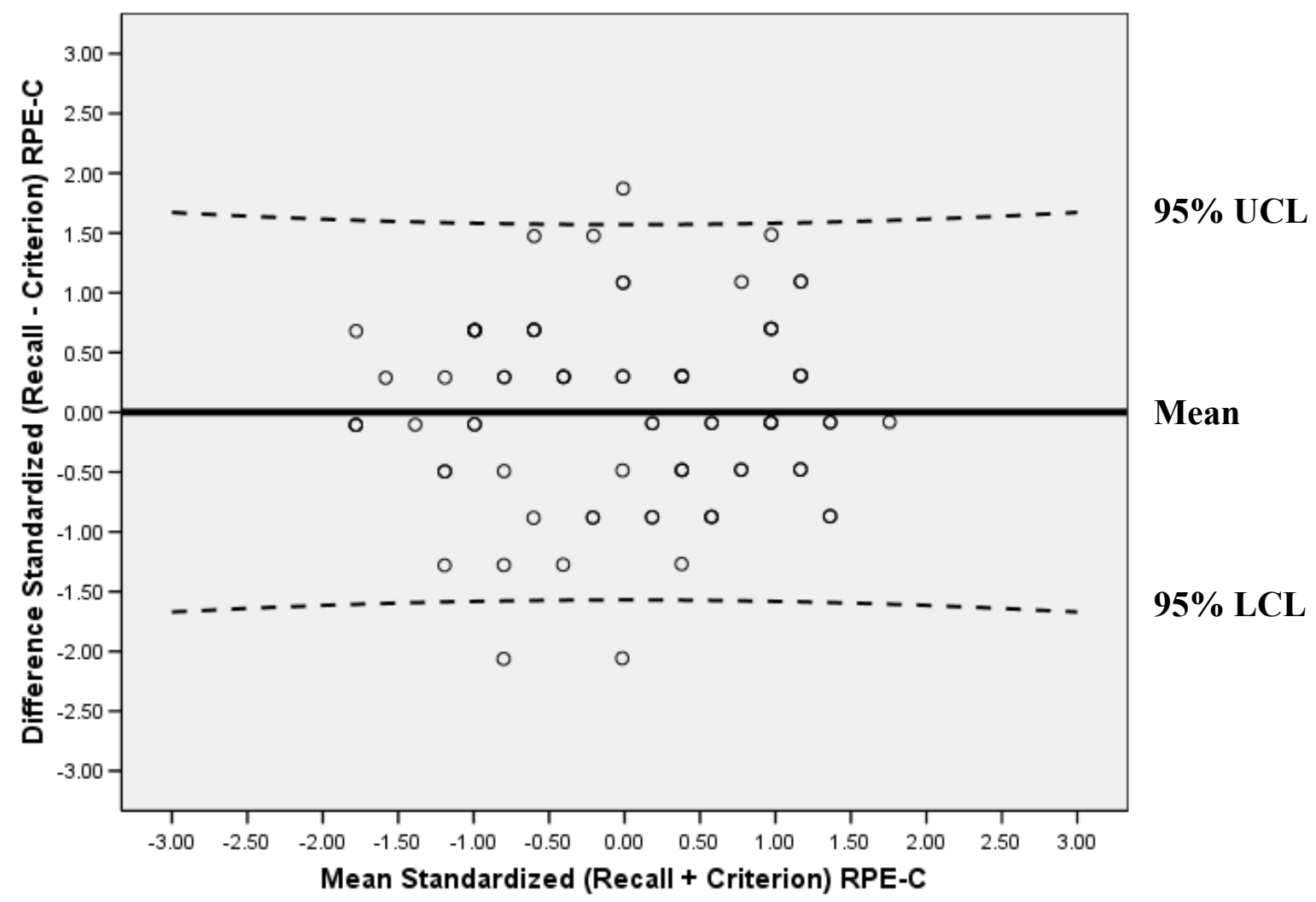

UCL: 95\% Upper Confidence Limit

LCL: 95\% Lower Confidence Limit 

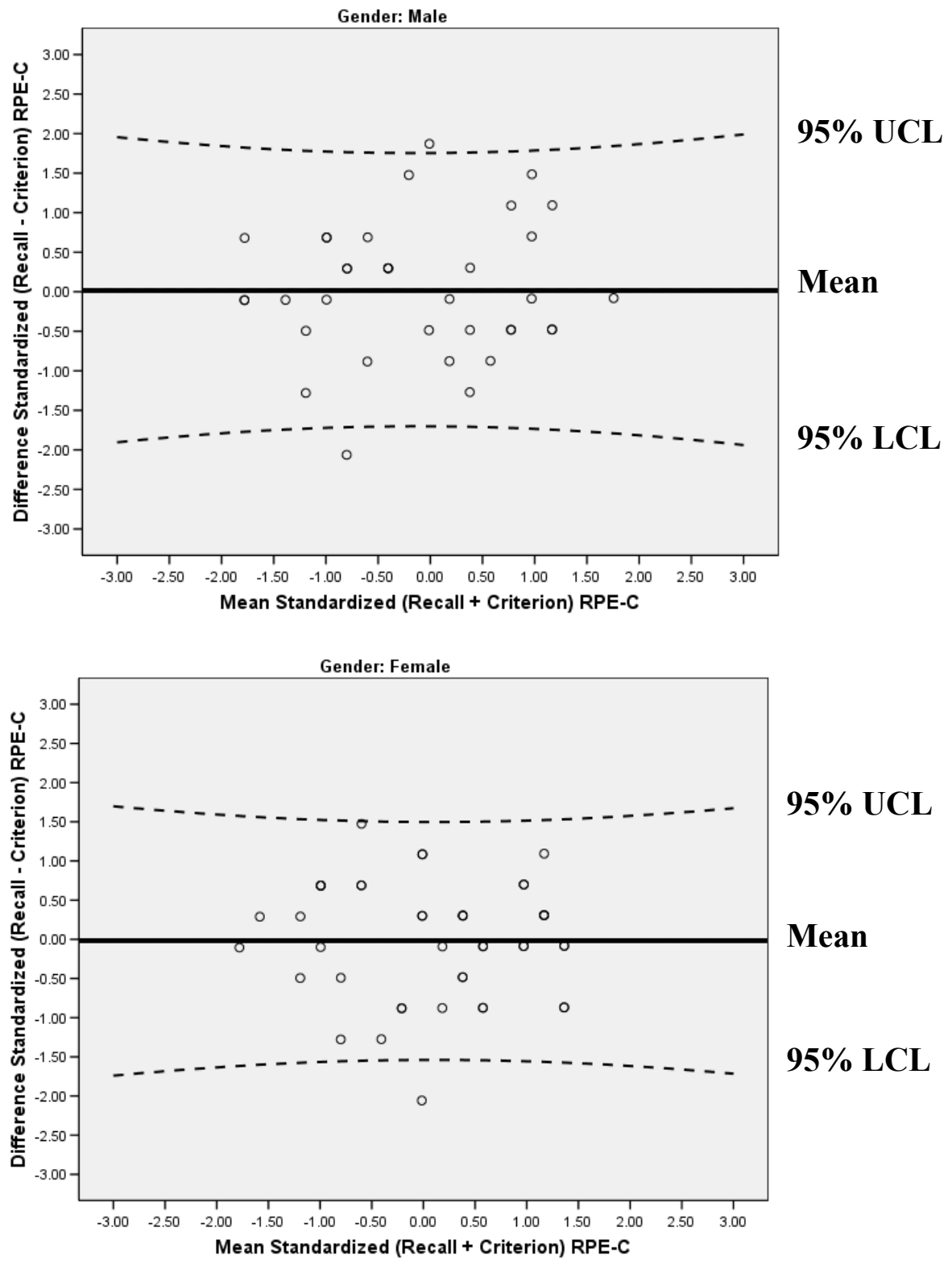

UCL: 95\% Upper Confidence Limit

LCL: 95\% Lower Confidence Limit 


\section{H.2 RESISTANCE EXERCISE}

\section{H.2.1 RPE-O}

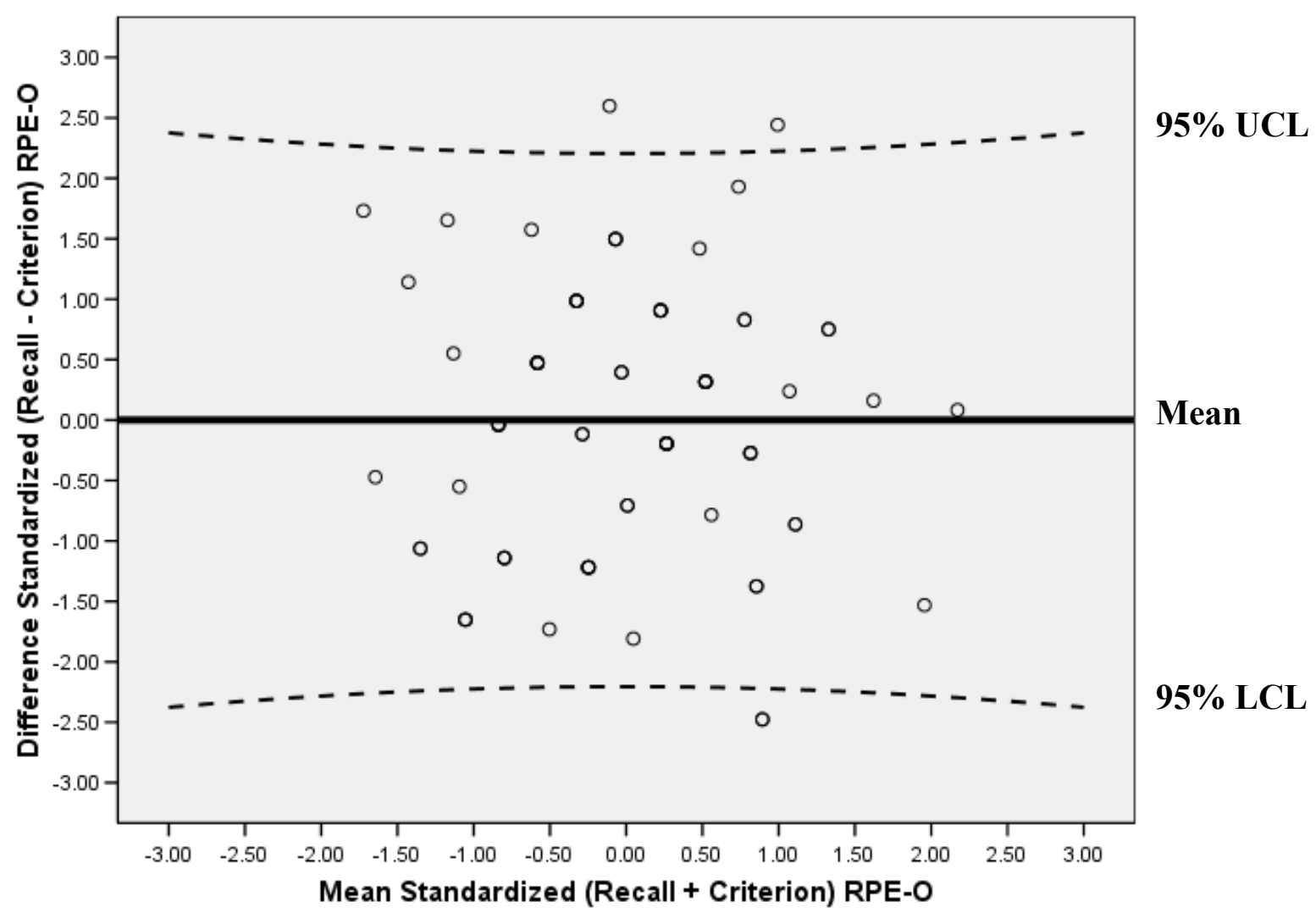

UCL: 95\% Upper Confidence Limit

LCL: 95\% Lower Confidence Limit 


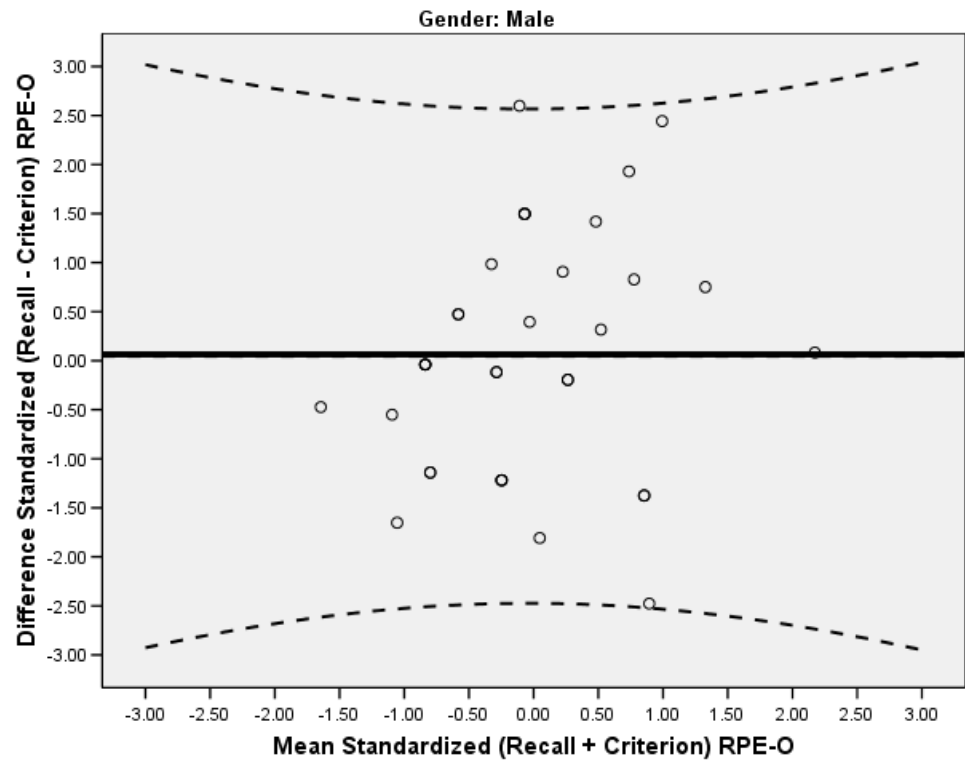

95\% UCL

Mean

95\% LCL

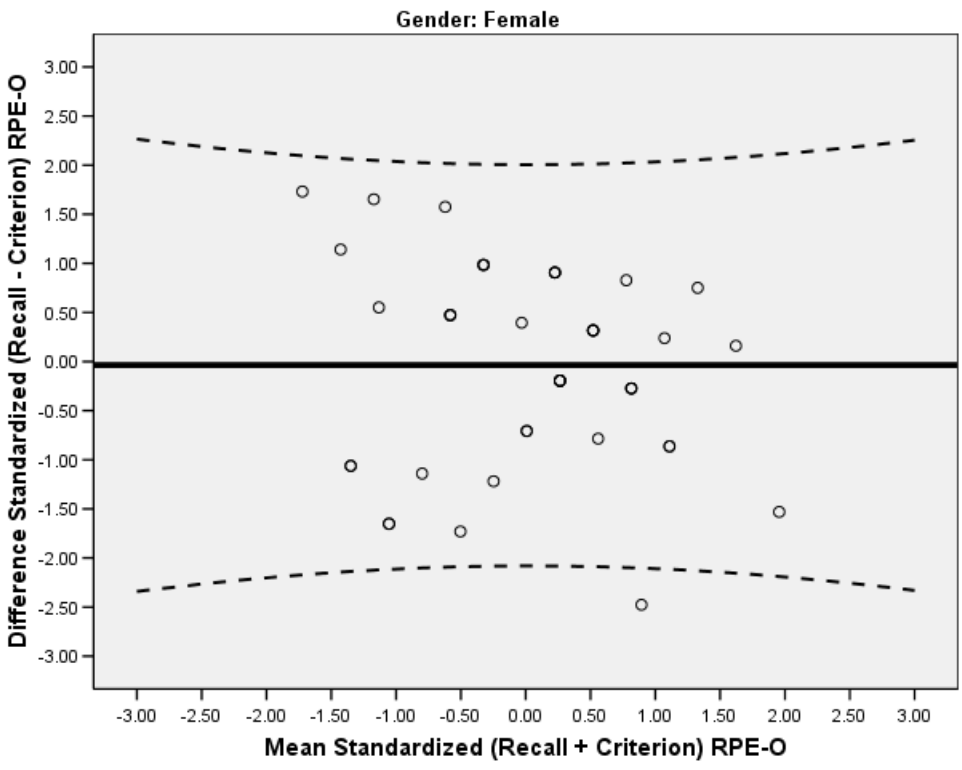

$95 \%$ UCL

Mean

$95 \%$ LCL

UCL: 95\% Upper Confidence Limit

LCL: 95\% Lower Confidence Limit 


\section{H.2.2 RPE-A}

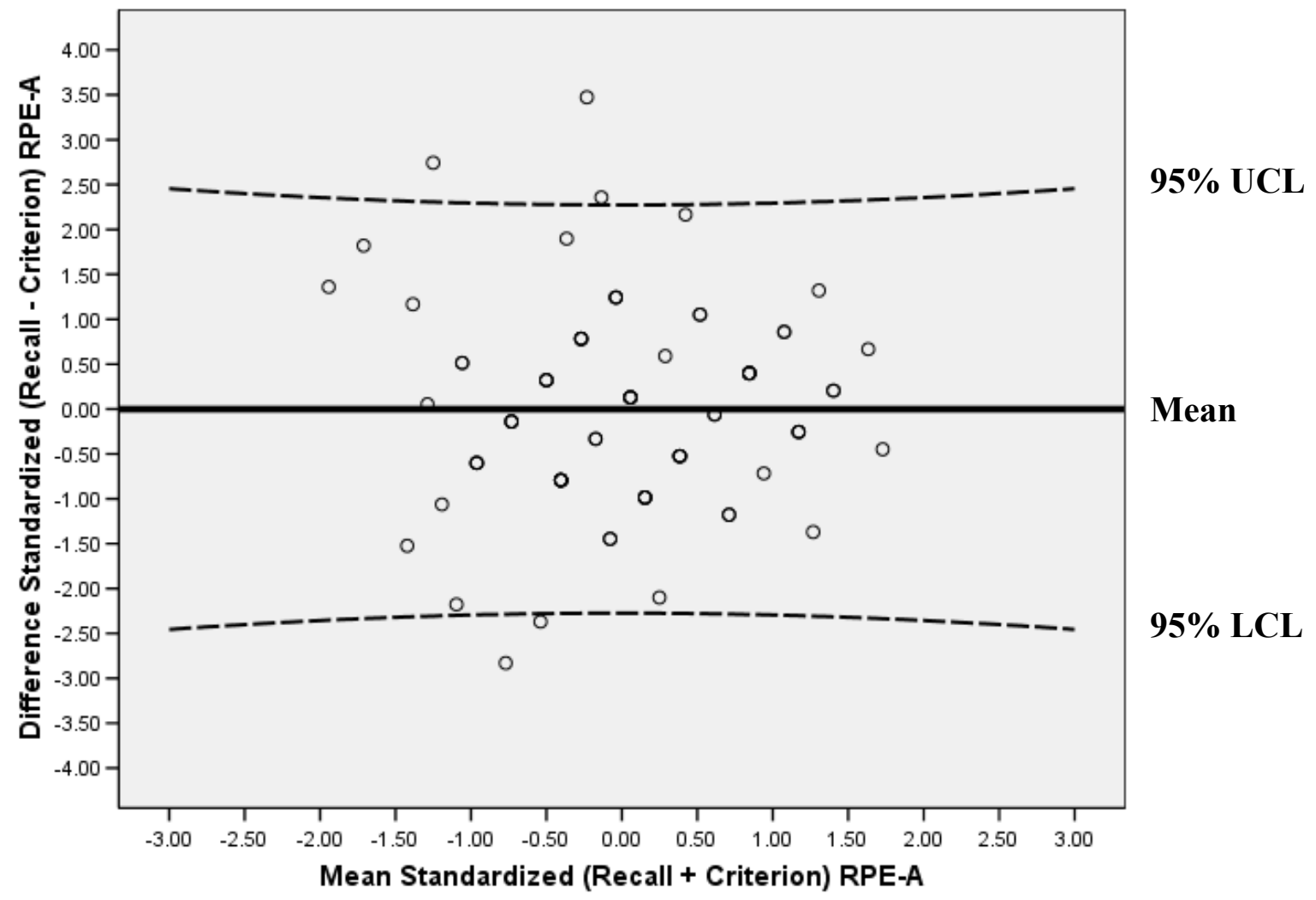

UCL: 95\% Upper Confidence Limit LCL: 95\% Lower Confidence Limit 


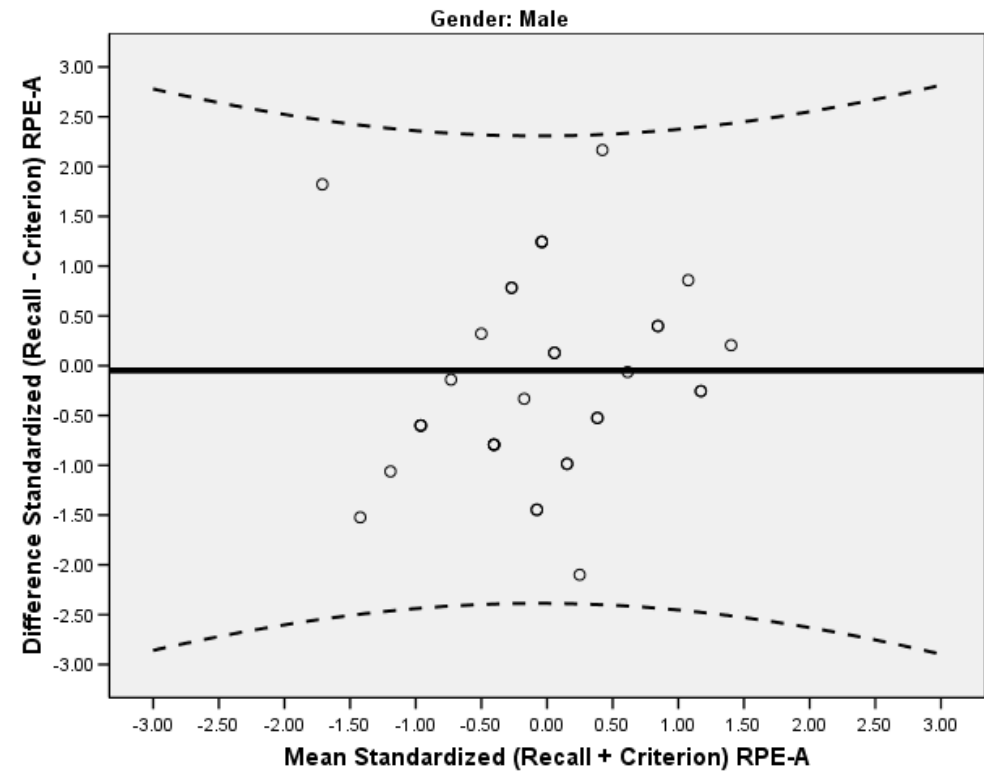

95\% UCL

Mean

95\% LCL

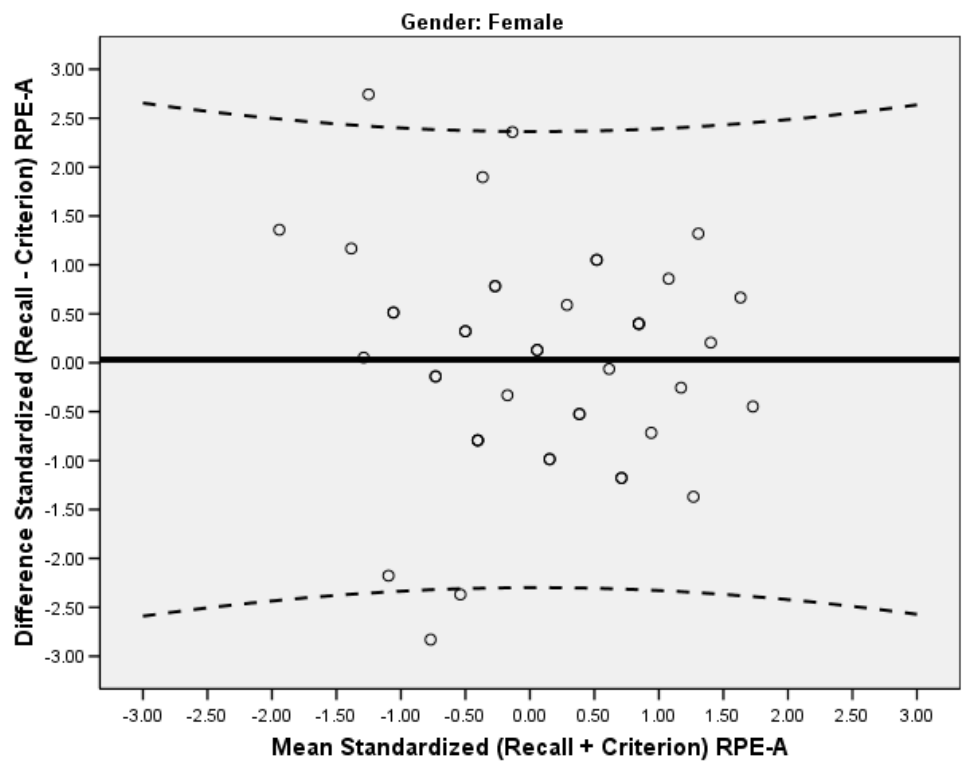

$95 \%$ UCL

Mean

95\% LCL

UCL: 95\% Upper Confidence Limit

LCL: 95\% Lower Confidence Limit 


\section{BIBLIOGRAPHY}

1. Lee I-M, Sesso HD, Oguma Y, and Paffenbarger RS Jr. Relative intensity of physical activity and risk of coronary heart disease. Circulation. Vol. 107: 1110-1116. 2003

2. Robertson RJ. Perceived Exertion for Practitioners: Rating Effort with the OMNI Picture System. Champaign, IL: Human Kinetics. 2004

3. Robertson RJ, Goss FL, Rutkowski J, et al. Concurrent validation of the OMNI perceived exertion scale for resistance exercise. Med Sci Sports Exerc. Vol. 35(2): 333-341. 2003

4. Robertson RJ, Goss FL, Dube J, et al. Validation of the adults OMNI scale of perceived exertion for cycle ergometer exercise. Med Sci Sports Exerc. Vol. 36(1): 102-108. 2004

5. Healthy People 1979. U.S. Surgeon General's Report on Health Promotion and Disease Prevention

6. United States of America Health Department of Human Service. Physical activity and health: A report of the Surgeon General. Atlanta, GA. 1996

7. American Cancer Society. http://www.cancer.org

8. Healthy People 2010. U.S. Surgeon General's Report on Health Promotion and Disease Prevention, http://www.healthypeople.gov

9. Centers for Disease Control (CDC) / National Institute of Health (NIH)

10. American College of Sports Medicine. Position Stand: The Recommended Quantity and Quality of Exercise for Developing and Maintaining Cardiorespiratory and Muscular Fitness in Healthy Adults. Med Sci Sports \& Exerc. Vol. 30(6):975-991. 1998

11. American Heart Association. http://www.americanheart.org

12. American Diabetic Association. http://www.diabetes.org

13. Welk, Gregory J. ed. Physical Activity Assessments for Health Related Research. Champaign, IL: Human Kinetics, 2002, pp. 21-27, 32, 108-120, 13-140, 153-158, 167175, 187-190, 206-208 
14. Sirard JR and Pate RP Physical Activity Assessment in Children and Adolescents. Sports Med. Vol. 3(6): 439-454. 2001

15. Noble BJ and Robertson RJ. Perceived Exertion. Champaign, IL: Human Kinetics. 1996

16. Powers SK and Howley ET. Exercise Physiology: Theory and Application to Fitness and Performance. $5^{\text {th }}$ ed. New York. McGraw-Hill. 2004

17. Bouchard C, Tremblay A, LeBlanc C, et al. A method to assess energy expenditure in children and adults. Am J Clin Nutr. Vol. 34: 461-467. 1983

18. Paffenbarger RS Jr, Wing AL, and Hyde RT. Physical activity as an index of heart attack risk in college alumni. Am. J. Epidemiol. Vol. 108: 161-175. 1978

19. Jacobs DR Jr, Hahn LP, Haskell WL, et al. Reliability and validity of a short physical activity history: CARDIA and the Minnesota Heart Health Program. J.Cardiopulm Rehab. Vol. 9: 448-459. 1989

20. Ainsworth BE, Haskell WL, Leon AS, et al. Compendium of physical activities: classification of energy costs of human physical activities. Med Sci Sports \& Exerc. Vol. 25: 71-80. 1993

21. Duncan GE, Sydeman SJ, Perri MG, et al. Can sedentary adults accurately recall the intensity of their physical activity? Prev Med. Vol. 33:18-26. 2001

22. Timperio A, Salmon J, and Crawford D. Validity and reliability of a physical activity recall instrument among overweight and non-overweight men and women. J Sci Med Sports. Vol. 6(4): 477-491. 2003

23. Strath SJ, Bassett DR, and Swartz AM. Comparison of the college alumnus questionnaire physical activity index with objective monitoring. Ann Epidemiol. Vol. 14:409-415. 2004

24. Ainsworth BE, Jacobs DR, Leon AS, et al. Accuracy of the College Alumnus Physical Activity Questionnaire. J Clin. Epidemiol. Vol. 46: 1403-1411. 1993

25. Jacobs DR, Ainsworth BE, Hartman TJ, and Leon AS. A simultaneous evaluation of 10 commonly used physical activity questionnaires. Med Sci Sports \& Exerc. Vol. 25: 81-91. 1993

26. Bonnefoy M, Normand S, Pachiaudi C, et al. Simultaneous validation of ten physical activity questionnaires in older men: a doubly labeled water study. J Am Geriatr Soc. Vol. 49:28-35. 2001

27. Rifas-Shiman SL, Gillman MW, Field AE, et al. Comparing physical activity questionnaires for youth: seasonal vs. annual format. Am J Prev Med. Vol. 20(4): 282285. 2001 
28. Singh PN, Fraser GE, Knutsen SF, et al. Validity of a physical activity questionnaire among African-American Seventh-day Adventists. Med Sci Sports \& Exerc. Vol. 33(3):468-475. 2001

29. Chasan-Taber L, Erickson JB, McBride JW, et al. Reproducibility of a self-administered lifetime physical activity questionnaire among female college alumnae. Am J Epidemiol. Vol. 155: 282-289. 2002

30. Sarkin JA, Nichols JF, Sallis JF, and Calfas KJ. Self-reports measures and scoring protocols affect prevalence estimates of meeting physical activity guidelines. Med Sci Sports \& Exerc. Vol. 32(1):149-156. 2000

31. Shephard RJ. Limits to the measurement of habitual physical activity by questionnaires: a review. Br J Sport Med. Vol. 37: 197-206. 2003

32. Borg G. Borg's Perceived Exertion and Pain Scales. Champaign, IL: Human Kinetics. 1998

33. Napolitano MA and Marcus BH. Targeting and tailoring physical activity information using print and information technologies. Exer Sport Sci Rev. Vol. 30(3): 122-128. 2002

34. Marcus BH, Owen N, Forsyth LH, Cavill NA, and Fridinger F. Physical activity interventions using mass media, print media, and information technology. Am $J$ Prev Med. Vol. 15(4):362-378. 1998

35. Kreuter MW, Oswald DL, Bull FC, and Clark EM. Are tailored health education materials always more effective than non-tailored materials? Health Education Research. Vol. 15(3): 305-315. 2000

36. Kreuter MW, Strecher VJ, and Glassman B. One size does not fit all: the case for tailoring print materials. Ann Behav Med. Vol. 21(4): 276-283. 1999

37. Bull FC, Kreuter MW, and Scharff DP. Effects of tailored, personalized and general health messages on physical activity. Patient Education \& Counseling. Vol. 36(2): 181192. 1999

38. Skinner JS, Hustler R, Bergsteinova V, and Buskirk ER. The validity and reliability of a rating scale of perceived exertion. Med Sci Sports \& Exerc. Vol. 5: 97-103. 1973

39. Stamford BA. The validity and reliability of subjective ratings of perceived exertion during work. Ergonomics. Vol. 19: 53-60. 1976

40. Gamberale F. Perceived exertion, heart rate, oxygen uptake and blood lactate in different work operations. Ergonomics. Vol. 15: 545-554. 1972

41. Hogan JC, Ogden GD, Gebhardt DL, and Fleishman EA. Reliability and validity of methods for evaluating perceived physical effort. J. Appl. Psychol. Vol. 65: 672-679. 1980 
42. Shephard RJ. Absolute versus relative intensity of physical activity in a dose-response context. Med Sci Sports Exerc. Vol. 33(6; Suppl): S400-S418. 2001

43. Kang J, Chaloupka EC, Mastrangelo MA, Donnelly MS, et al. Regulating exercise intensity using ratings of perceived exertion during arm and leg ergometry. Eur J Appl Physiol. Vol. 78: 241-246. 1998

44. Robertson RJ, Goss FL, Boer NF, et al. Children's OMNI Scale of perceived exertion: Mixed gender and race validation. Med Sci Sports Exer. Vol. 32: 452-458. 2000

45. Utter AC, Robertson RJ, Nieman D, and Kang J. Children's OMNI scale of perceived exertion: Walking/Running evaluation. Med Sci Sports Exer. Vol. 34: 139-144. 2002

46. Dannecker EA, Price DD, and Robinson ME. An examination of the relationships among recalled, expected, and actual intensity and unpleasantness of delayed onset muscle pain. J Pain. Vol. 4(2): 74-81. 2003

47. Dunbar CC, and Kalinski MI. Using RPE to regulate intensity during a 20-week training program for postmenopausal women: a pilot study. Percep Motor Skills. Vol. 99(2): 688690. 2004

48. Robbins LB, Pender NJ, Ronis DL, et al. Physical activity, self-efficacy, and perceived exertion among adolescents. Res Nurs Health. Vol. 27: 435-446. 2004

49. Shigematsu R, Ueno LM, Nakagaichi M, et al. Rate of perceived exertion as a tool to monitor cycling exercise intensity in older adults. J Aging Phys Activ. Vol. 12(1): 3-9. 2004

50. Woods S, Bridge T, Nelson, et al. The effects of rest internal length on ratings of perceived exertion during dynamic knee extension exercise. J Strength Cond Res. Vol. 18(3): 540-545. 2004

51. Robertson RJ, Goss FL, Aaron D, et al. Observation of Perceived Exertion in Children using the OMNI Pictorial Scale. Med Sci Sports Exer. December 2005

52. Foster C, Hector LL, Welsh R, et al. Effects of specific versus cross-training on running performance. Eur J Appl Physiol. Vol. 70: 367-372. 1995

53. Foster C, Florhaug JA, Frankling J, et al. A new approach to monitoring exercise training. J Strength Cond Res. Vol. 15(1): 109-115. 2001

54. McGuigan MR and Foster C. A new approach to monitoring resistance training. $J$ Strength Cond Res. Vol. 16(6): 42-47. 2004

55. Sweet TW, Foster C, McGuigan MR, and Brice G. Quantitation of resistance training using the session rating of perceived exertion method. J Strength Cond Res. Vol. 18(4): 796-802. 2004 
56. Serrano MA, Salvador A, Gonzalez-Bono, E, et al. Relationships between recall of perceived exertion and blood lactate concentration in a judo competition. Percep Motor Skills. Vol. 92:1139-1148. 2001

57. American College of Sports Medicine. ACSM's Guidelines for Exercise Testing and Prescription $-7^{\text {th }}$ Ed. Lippincott Williams \& Wilkins. 2005

58. Lagally KM, and Costigan EM. Anchoring procedures in reliability ratings of perceived exertion during resistance exercise. Percep Motor Skills. Vol. 98(3 Pt 2):1285-1295. 2004

59. Gearhart RF, Goss FL, Lagally KM, et al. Standardized scaling procedures for rating perceived exertion during resistance exercise. J Strength Cond Res. Vol. 15(3):320-325. 2001

60. Rudra CB, Williams MA, Lee IM, Miller RS, and Sorensen TK. Perceived exertion during prepregnancy physical activity and preeclampsia risk. Med Sci Sports Exerc. Vol. 37:1836-1841, 2005.

61. Rudra CB, Williams MA, Lee IM, Miller RS, and Sorensen TK. Perceived exertion in physical activity and risk of gestational diabetes mellitus. Epidemiology. Vol. 17:31-37, 2006.

62. Schafer MS, Thekkada S, Gallagher Jr. M, Hunt S, Aaron DJ, Goss FL, and Robertson RJ. Validation of the OMNI RPE Seven Day Effort Recall Questionnaire. Medicine and Science in Sports and Exercise. Vol. 38 (5), Supplement, S79, 2006

63. Hoffman MD, Kassay KM, Zeni AI, and Clifford PS. Does the Amount of Exercising Muscle Alter the Aerobic Demand of Dynamic Exercise? Eur J Appl Physiol Occup Physiol. Vol. 74(6):541-547. 1996

64. Franke WD, Boettger CF, and McLean SP. Effects of Varying Central Command and Muscle Mass on the Cardiovascular Responses to Isometric Exercise. Clinical Physiology. Vol. 20(5):380-387. 2000

65. Thekkada S, Krause M, McGrane M, Shouey L, Aaron DJ, Goss FL and Robertson RJ. Validation of Session RPE for a Load Incremented Cycle Ergometer Protocol. Medicine and Science in Sports and Exercise. Vol. 39 (5), Supplement, 2007

66. Thekkada S, Ghigiarelli JJ, Schafer MA, Goss FL, Aaron DJ, and Robertson RJ. Validation of an OMNI RPE based 7-day recall questionnaire to assess session RPE. Medicine and Science in Sports and Exercise. Vol. 38 (5), Supplement, 2006

67. Gearhart RF, Goss FL, Lagally KM, et al. Ratings of Perceived Exertion in Active Muscle during High-Intensity and Low-Intensity Resistance Exercise. J Strength Cond Res. Vol. 16(1):87-91. 2002

68. Egan AD. Session Rating of Perceived Exertion During High Intensity and Low Intensity Bouts of Resistance Exercise. UW-L Journal of Undergraduate Research VI. 2003 